ANALYSIS \& PDE Volume 6 No.1 2013 (20) ROBERT J. BERMAN

\title{
RELATIVE KÄHLER-RICCI FLOWS AND THEIR QUANTIZATION
}




\title{
RELATIVE KÄHLER-RICCI FLOWS AND THEIR QUANTIZATION
}

\author{
ROBERT J. BERMAN
}

Let $\pi: \mathscr{L} \rightarrow S$ be a holomorphic fibration and let $\mathscr{L}$ be a relatively ample line bundle over $\mathscr{X}$. We define relative Kähler-Ricci flows on the space of all Hermitian metrics on $\mathscr{L}$ with relatively positive curvature and study their convergence properties. Mainly three different settings are investigated: the case when the fibers are Calabi-Yau manifolds and the case when $\mathscr{L}= \pm K_{\mathscr{X} / S}$ is the relative (anti)canonical line bundle. The main theme studied is whether "positivity in families" is preserved under the flows and its relation to the variation of the moduli of the complex structures of the fibers. The "quantization" of this setting is also studied, where the role of the Kähler-Ricci flow is played by Donaldson's iteration on the space of all Hermitian metrics on the finite rank vector bundle $\pi_{*} \mathscr{L} \rightarrow S$. Applications to the construction of canonical metrics on the relative canonical bundles of canonically polarized families and Weil-Petersson geometry are given. Some of the main results are a parabolic analogue of a recent elliptic equation of Schumacher and the convergence towards the Kähler-Ricci flow of Donaldson's iteration in a certain double scaling limit.

1. Introduction

2. The general setting

3. The Calabi-Yau setting

148

4. The (anti)canonical setting

\section{Introduction}

1A. Background. On an $n$-dimensional Kähler manifold $\left(X, \omega_{0}\right)$ Hamilton's Ricci flow [Hamilton 1982] on the space of Riemannian metrics on $X$ preserves the Kähler condition of the initial metric and may be written as the Kähler-Ricci flow

$$
\frac{\partial \omega_{t}}{\partial t}=-\operatorname{Ric} \omega_{t}
$$

When $X$ is a Calabi-Yau manifold (which here will mean that the canonical line bundle $K_{X}$ is holomorphically trivial) it was shown by Cao [1985] that the corresponding flow in the space of Kähler metrics in $\left[\omega_{0}\right] \in H^{2}(X, \mathbb{R})$ has a large time limit. The limit is thus a fixed point of the flow which coincides with the unique Ricci flat Kähler metric in $\left[\omega_{0}\right]$, whose existence was first established by Yau [1978] in his celebrated proof of the Calabi conjecture. The non-Calabi-Yau cases when $\left[\omega_{0}\right]$ is the first Chern class $c_{1}(L)$ of $L=r K_{X}$, where $r= \pm 1$, have also been studied extensively (where $-r \omega$ is added to the right side in (1-1)). In general the fixed points of the corresponding Kähler-Ricci flows are hence

MSC2010: 14J32, 32G05, 32Q20, 53C55.

Keywords: Kähler-Ricci flow, positivity, Kähler-Einstein metric, balanced metric, Weil-Petersson metric. 
Kähler-Einstein metrics of negative $(r=1)$ and positive $(r=-1)$ scalar curvature. The convergence towards a fixed point — when it exists — in the latter positive case (i.e., $X$ is a Fano manifold) was only established very recently by Perelman (unpublished) and by Tian and Zhu [2007].

A distinctive feature of Kähler geometry is that a Kähler metric $\omega$ may be locally described in terms of a local function $\phi$, such that $\omega=d d^{c} \phi$. In the integral case, that is, when $\left[\omega_{0}\right]=c_{1}(L)$ is the first Chern class of an ample line bundle $L \rightarrow X$, this just amounts to the global fact that the space of Kähler metrics $\omega$ in $c_{1}(L)$ may be identified with the space $\mathscr{H}_{L}$ of smooth metrics $h$ on the line bundle $L$ with positive curvature form $\omega$, modulo the action of $\mathbb{R}$ on $\mathscr{H}_{L}$ by scalings. Locally, $h=e^{-\phi}$ and we will refer to the additive object $\phi$ as a weight on $L$ (see Section 2A). In this notation the Kähler-Einstein equations may be expressed as Monge-Ampère equations on $\mathscr{H}_{L}$. For example, on a Calabi-Yau manifold $\omega_{\phi}:=d d^{c} \phi$ is Ricci flat precisely when

$$
\left(d d^{c} \phi\right)^{n} / n !=\mu
$$

where $\mu$ is the canonical probability measure on $X$ such that $\mu=i^{n^{2}} \Omega \wedge \bar{\Omega}$, for $\Omega$ a suitable global holomorphic $n$-form trivializing $K_{X}$ (to simplify the notion we will in the following always assume that the volume of the given class $\left[\omega_{0}\right]$ is equal to one, so that $\omega_{0}^{n} / n !$ defines a probability measure on $X$ for any $\left.\omega \in\left[\omega_{0}\right]\right)$. By letting $\mu$ depend on $\phi$ in a suitable way general Kähler-Einstein metrics are obtained.

As emphasized by Yau [1987] one can expect to obtain approximations to Kähler-Einstein metrics by using holomorphic sections of high powers of a line bundle. In this direction Donaldson [2009] introduced certain iterations on the "quantization" (at level $k$ ) of the space $\mathscr{H}_{L}$ of Kähler metrics in $c_{1}(L)$. Geometrically, this quantized space, denoted by $\mathscr{H}^{(k)}$, is the space of all Hermitian metrics on the finitedimensional vector space $H^{0}(X, k L)$ of global holomorphic sections of $k L$, where $k L$ denotes the $k$-th tensor power of $L$, in our additive notation (for the definition see Section 2D). In other words $\mathscr{H}^{(k)}$ can be identified with the symmetric space $\operatorname{GL}\left(N_{k}, \mathbb{C}\right) / U\left(N_{k}\right)$ of $N_{k} \times N_{k}$ Hermitian matrices which in turn, using projective embeddings, corresponds to the space of level $k$ Bergman metrics on $L$. The fixed points of Donaldson's iteration are called balanced metrics at level $k$ (with respect to $\mu$ ) and they first appeared in the previous work of Bourguignon, Li, and Yau [Bourguignon et al. 1994]. Again, in the $\pm K_{X}$-setting one lets $\mu$ depend on $\phi$ in a suitable way leading to different settings (see below). In the limit when $L$ is replaced by a large tensor power it has very recently been shown that balanced metrics in the different settings indeed converge to Kähler-Einstein metrics [Wang 2005; Keller 2009; Berman et al. 2009]. It was pointed out in [Donaldson 2009] that it seems likely that these iterations can be viewed as discrete approximations of the Ricci flow. This will be made precise and confirmed in the present paper (Theorem 3.15 and Theorem 4.18).

1B. Outline of the present setting and the main results. The aim of the present paper is to study relative versions of the Kähler-Ricci flow and Donaldson's iteration (in the various settings). More precisely, the geometric setting is that of a holomorphic fibration $\pi: \mathscr{X} \rightarrow S$ of relative dimension $n$ and a relatively ample line bundle $\mathscr{L} \rightarrow \mathscr{L}$. The fibration will mainly be assumed to be a proper submersion over a connected base, so that all fibers are diffeomorphic (for general quasiprojective morphisms see Section 4E). Note 
that in applications $S$ typically arises as a moduli space or Teichmüller space and $\mathscr{X}$ as the corresponding universal family.

The main points that will be considered are

- the question whether "positivity in families" is preserved under the flows;

- the convergence of the "quantized" (finite-dimensional) setting of Donaldson's iteration towards the Kähler-Ricci flow setting in the "semiclassical" limit (i.e., the large $k$-limit).

More precisely, denote by $\mathscr{H}_{\mathscr{L} / S}$ the space of all metrics on $\mathscr{L}$ which are fiberwise of positive curvature. In other words, $\mathscr{H}_{\mathscr{L} / S}$ is an infinite-dimensional fiber bundle over $S$ whose fibers are of the form $\mathscr{H}_{L}$, as in the previous section. The relative Kähler-Ricci flows are defined as suitable flows on $\mathscr{H}_{\mathscr{L} / S}$ such that the induced flow of curvature forms restricts to the usual Kähler-Ricci flow fiberwise: we will say that "positivity is preserved under the flow" if, for any initial metric with positive curvature (in all directions on $\mathscr{X}$ ), the evolved metric also has positive curvature for all times; that is, the flow induces a flow of Kähler forms on the total space $\mathscr{L}$ of the fibration (and not only along the fibers).

As will be explained below, the two points above are closely related. For example, the preservation of positivity in the relative Kähler-Ricci flow setting can be seen as a limiting version of the well-known positivity of direct image bundles in the quantized setting (the latter positivity is a fundamental tool in complex geometry; see [Kawamata 1982; Berndtsson 2009a], for example). As another application of the convergence in the second point above (in the absolute case when $S$ is a point) we will deduce the uniform convergence of Donaldson's canonically balanced metrics from the well-known convergence of the Kähler-Ricci flow (Theorem 4.20).

The Calabi-Yau setting. Let us first summarize the main results in the setting when the fibers are CalabiYau. It should however be stressed that the setting when the fibers are canonically polarized appears to be the one most suited for geometric applications (see below). In the Calabi-Yau setting flow $\phi_{t}$ in $\mathscr{H}_{\mathscr{L} / S}$ is defined fiberwise by

$$
\frac{\partial \phi_{t}}{\partial t}=\log \frac{\left(d d^{c} \phi_{t}\right)^{n} / n !}{\mu},
$$

with $\mu$ a measure as in (1-2). Of course, adding the pull-back of a time-dependent function on the base $S$ to the right side of the previous equation does not alter the induced flows of the fiberwise restricted Kähler forms $d_{X} d_{X}^{c} \phi_{t}$, but it certainly effects the flow of $d d^{c} \phi_{t}$ on $\mathscr{X}$ which will typically not preserve the initial Kähler property.

One of the main results of the present paper is a parabolic evolution equation along the flow (1-3) for the function

$$
c(\phi):=\frac{1}{n}\left(d d^{c} \phi\right)^{n+1} /\left(d_{X} d_{X}^{c} \phi\right)^{n} \wedge i d s \wedge d \bar{s}
$$

on $\mathscr{X}$ which is well-defined when $S$ is embedded in $\mathbb{C}$. The point is that $c(\phi)>0$ precisely when $d d^{c} \phi>0$ on $\mathscr{X}$. The evolution equation for $c\left(\phi_{t}\right)$ reads (Theorem 3.3)

$$
\left(\frac{\partial}{\partial t}-\Delta_{\omega_{t}^{X}}\right) c\left(\phi_{t}\right)=\left|A_{\omega_{t}}\right|_{\omega_{t}^{X}}^{2}-\omega_{\mathrm{WP}}
$$


where $\omega_{t}^{X}$ denotes the flow of the fiberwise restricted curvature forms, $A_{\omega_{t}}$ is a certain representative of the Kodaira-Spencer class of the fiber $\mathscr{X}_{S}$ and $\omega_{\mathrm{WP}}$ is the pull-back to $\mathscr{X}$ of the (generalized) Weil-Petersson form on the base $S$; by a result of Tian [1987] and Todorov [1989], which we will reprove, $\omega_{\mathrm{WP}}$ can be represented by the global squared $L^{2}$-norm of $A_{\omega_{\mathrm{KE}}}$ for $\omega_{\mathrm{KE}}$ the unique Ricci flat metric in $c_{1}(L)$. Applying the maximum principle then gives (Corollary 3.4) that the initial condition $d d^{c} \phi_{0}>0$ implies that

$$
d d^{c} \phi_{t}>-t \omega_{\mathrm{WP}}
$$

(and similarly when the initial curvature is semipositive). By its very definition $\omega_{\mathrm{WP}}$ vanishes at $s$ precisely when the infinitesimal deformation of the complex structure on the fibers $\mathscr{L}_{s}$ (i.e., the KodairaSpencer class) vanishes at $s$. In particular, if the fibration $\pi: \mathscr{L} \rightarrow S$ is holomorphically trivial, then, by inequality (1-5), positivity is indeed preserved along the flow. This latter situation appears naturally in Kähler geometry. Indeed, if the base $S$ is an annulus in $\mathbb{C}$ and $\phi_{S}$ is rotationally invariant, then $\phi_{S}$ corresponds to a curve in $\mathscr{H}_{L}$ and $c\left(\phi_{S}\right)$ is then the geodesic curvature of the curve $\phi_{s}$ when $\mathscr{H}_{L}$ is equipped with its symmetric space Riemannian metric (see [Chen 2000] and references therein). In the nonnormalized $K_{X}$-setting (see Section 4) the equation (1-4) can be seen as a parabolic generalization of a very recent elliptic equation of Schumacher [2008].

Similarly, the "quantized" version of the previous setting is studied, that is, the relative version of Donaldson's iteration. It gives an iteration on the space of all Hermitian metrics $H$ on the finite rank vector bundle $\pi_{*} k \mathscr{L} \rightarrow S$ for any positive integer $k$ (recall that the fiber of $\pi_{*} \mathscr{L}$ over $s$ is, by definition, the space $H^{0}\left(\mathscr{L}_{S}, \mathscr{L}_{S}\right)$ of all global holomorphic sections on the fiber $\mathscr{L}_{s}$ with values in $\left.\mathscr{L}_{\mid \mathscr{X}_{s}}\right)$. More precisely, we will study the equivalent fiberwise iteration $\phi_{m}^{(k)}$ in $\mathscr{H}_{\mathscr{L} / S}$ obtained by applying the (scaled) Fubini-Study map to Donaldson's iteration. It will be called the relative Bergman iteration at level $k$. When the discrete time $m$ tends to infinity it is shown (Theorem 3.9) that the iteration converges to a fiberwise balanced weight:

$$
\phi_{m}^{(k)} \rightarrow \phi_{\infty}^{(k)}
$$

in the $\mathscr{C}^{\infty}$-topology on $\mathscr{X}_{s}$, uniformly with respect to $s$. It is also observed that an analogue of the inequality (1-5) holds; that is,

$$
d d^{c} \phi_{m}^{(k)} \geq-\frac{k}{m} \omega_{\mathrm{WP}}
$$

This turns out to be a simple consequence of a recent theorem of Berndtsson [2009a] about the curvature of vector bundles of the form $\pi_{*}\left(\mathscr{L}+K_{\mathscr{L} / S}\right)$. We also confirm Donaldson's expectation about the semiclassical limit when the level $k$ tends to infinity. More precisely, it is shown that, in the double scaling limit where $m / k \rightarrow t$, the (relative) Bergman iteration at level $k$ approaches the (relative) Kähler-Ricci flow (1-3):

$$
\phi_{m}^{(k)} \rightarrow \phi_{t}
$$

uniformly on $\mathscr{X}$. In particular, combining this convergence with (1-6) gives an alternative proof of the semipositivity in the inequality (1-5). Moreover, by taking $m=m_{k}$ such that $m / k \rightarrow \infty$ this gives a dynamical construction of solutions to the inhomogeneous Monge-Ampère equation (1-2) in the setting where $\mu$ is any fixed volume form (Corollary 3.16). 
The (anti)canonical setting. The previous results also have analogues in the setting when the ample line bundle $\mathscr{L}$ is either the relative canonical line bundle $K_{\mathscr{L} / S}$ over $\mathscr{L}$ or its dual, which we write as $\mathscr{L}= \pm K_{\mathscr{L} / S}$ in our additive notation. The starting point is the fact that any metric $h=e^{-\phi}$ on $\pm K_{X}$ induces, by the very definition of $K_{X}$, a volume form on $X$ which may be written suggestively as $e^{ \pm \phi}$. The previous constructions, that is, the relative Kähler-Ricci flows and the Donaldson iteration, can then be repeated word for word for these $\phi$-dependent measures $\mu=\mu(\phi)$. For example, the relative Kähler-Ricci flows are defined by

$$
\frac{\partial \phi_{t}}{\partial t}=\log \left(\frac{\left(d d^{c} \phi_{t}\right)^{n} / n !}{e^{ \pm \phi_{t}}}\right)
$$

and we obtain (Theorem 4.7) a corresponding parabolic equation for $c\left(\phi_{t}\right)$ :

$$
\left(\frac{\partial}{\partial t}-\left(\Delta_{\omega_{t}^{X}}- \pm 1\right)\right) c\left(\phi_{t}\right)=\left|A_{\omega_{t}}\right|_{\omega_{t}^{X}}^{2},
$$

and as a consequence the flows always preserve positivity (Corollary 4.9) in these settings. In fact, in the case of infinitesimally nontrivial fibration the flows will even improve the positivity; that is, any initial weight which is merely semipositively curved instantly becomes positively curved under the flows. In the $+K_{X}$-setting the unique fixed point of the flow (1-8) is the (fiberwise) normalized Kähler-Einstein weight uniquely determined by

$$
e^{-\phi_{\mathrm{KE}}}=\left(\omega_{\mathrm{KE}}\right)^{n} / n !
$$

where $\omega_{\mathrm{KE}}$ is the unique Kähler-Einstein metric on $X$ (Corollary 4.3). The corresponding elliptic equation for $c\left(\phi_{\mathrm{KE}}\right)$ was first obtained by Schumacher [2008] who used it to deduce the following interesting result: $\phi_{\mathrm{KE}}$ is always semipositively curved on the total space of $\mathscr{L}$ and strictly positively curved for an infinitesimally nontrivial fibration. As a consequence he obtained several applications to the geometry of moduli spaces. For example, applied to the case when $\mathscr{X} \rightarrow S$ is the universal curve over the Teichmüller space of Riemann surfaces of genus $g \geq 2$ it gives, when combined with Berndtsson's theorem (Theorem 3.10), a new proof of the hyperbolicity result of Liu, Sun, and Yau [Liu et al. 2008] saying that the curvature of the Weil-Petersson metric on the Teichmüller space is dual Nakano positive.

In the $-K_{X}$-setting the relative Kähler-Ricci flow will diverge for generic initial data. But using the convergence on the level of Kähler forms, established by Perelman and Tian and Zhu, will show that, if the Fano manifold $X$ admits a unique positively curved Kähler-Einstein metric $\omega_{\mathrm{KE}}$, the flow does converge to a weight for $\omega_{\mathrm{KE}}$ in the normalized $\pm K_{X}$-setting. This latter setting is simply obtained by normalizing the volume forms $e^{ \pm \phi}$ used above.

We will also use the relative Bergman iteration to obtained a "quantized" version of Schumacher's result: the canonical "semibalanced" metric at level $k$ on $K_{\mathscr{X} / S}$, which by definition is fiberwise normalized and balanced, is smooth with semipositive curvature on $\mathscr{X}$ (Corollary 4.16) and strictly positively curved in the case of an infinitesimally nontrivial fibration. As a consequence the semibalanced metric gives an alternative to the canonical metric on $k K_{\mathscr{L} / S}$ introduced in [Narasimhan and Simha 1968] (see also Kawamata 1982; Tsuji 2011; Berndtsson and Păun 2008a for positivity properties of this latter metric). 
In Section 4E some of the results concerning the setting when $K_{X}$ is ample are generalized to projective fibrations of varieties of general type (i.e., $K_{X}$ is merely big) and the corresponding canonical semibalanced metric is shown to have a positive curvature current (Theorem 4.21). Relations to deformation invariance of plurigenera [Siu 1998] are also briefly discussed.

1C. Further relations to previous results. A variant of Donaldson iteration (but with a single parameter $k$ ) in the $K_{X}$-setting was introduced by Tsuji [2006]. He proved convergence in the $L^{1}$-topology towards the normalized Kähler-Einstein weight $\phi_{\mathrm{KE}}$ in the large $k$-limit (see [Song and Weinkove 2010] for a proof of uniform convergence) and deduced the semipositivity result for $\phi_{\mathrm{KE}}$ of Schumacher referred to above. These works of Tsuji and Schumacher provided an important motivation for the present one. Steve Zelditch has also informed the author of a joint work in progress with Jian Song, where they show that the linearization of Tsuji's iteration at the fixed point coincides with the linearization of the Kähler-Ricci flow. It should also be pointed out that another discretization of the Kähler-Ricci flow on a Fano manifold was studied by Rubinstein [2008] and Keller [2009].

The $C^{0}$-convergence of the Bergman iteration at a fixed level $k$ in the Calabi-Yau setting (or more generally in the setting of a fixed measure $\phi$ ) was pointed out by Donaldson [2009] and the proof was sketched. Sano [2006] provided an explicit proof in the constant scalar curvature setting (see Section 4F).

It is also interesting to compare with the very recent work of Fine [2010] concerning the constant scalar curvature setting. He shows that a continuous version of Donaldson's iteration in this latter setting, called balancing flows, converges to the Calabi flow, when the latter flow exists. Julien Keller and Huai-Dong Cao have informed the author of a joint work in progress where an analogue of Fine's balancing flows in the Calabi-Yau setting (or more generally in the setting of a fixed volume form $\mu$ ) is shown to converge to a flow on metrics, which however is different than the Kähler-Ricci flow.

There are also, at last tangential, relations to the work of Gross and Wilson [2000], where fibrations with Calabi-Yau fibers are considered. In particular, they construct certain semiflat Kähler metrics $\omega$ on the fibration $\mathscr{X}$; that is, $\omega$ is fiberwise Ricci flat. Such metrics first appeared in the string theory literature [Greene et al. 1990]. In this terminology the inequality (1-5) shows that the relative Kähler-Ricci flow deforms any given Kähler metric to a semiflat one, when there is no variation of the moduli of the complex structure of the fibers. More generally, this latter statement holds in a double scaling limit when the variation of the complex structure is very small in the sense that $\omega_{\mathrm{FS}}\left(s_{t}\right) t \rightarrow 0$ as $t \rightarrow \infty$.

A Kähler-Ricci flow on compact fibrations $\mathscr{X}$ with Calabi-Yau fibers was also considered recently by Song and Tian [2012]. But they consider the usual (i.e., nonrelative) Kähler-Ricci flow (with $r=1$ ) when the canonical line bundle is only semiample and relatively trivial (i.e., the base $S$ is the canonical model of $\mathscr{X}$ ). They prove that the flow collapses the fibers so that the limit is the pull-back of metric on the base $S$ solving a "twisted" Kähler-Einstein equation where the twist is described by the (generalized) Weil-Petersson form $\omega_{\mathrm{FS}}$.

1D. Organization of the paper. In Section 2 a general setting is introduced and the associated relative Kähler-Ricci flow and its quantization are defined. General convergence criteria for the flows are given. In the following two sections the general setting is applied to get convergence results in particular settings 
of geometric relevance: the Calabi-Yau setting (Section 3) and the (anti)canonical setting (Section 4). The new feature of these convergence results for the Kähler-Ricci flows is that the convergence takes place on the level of weights, that is, for the potentials of the evolving Kähler metrics. Furthermore, the main question whether "positivity in families" is preserved under the flows is studied in these two sections and relations to Weil-Petersson geometry are also discussed. It is also shown that the quantized flows converge to Kähler-Ricci flows in the large tensor power limit. Applications to canonical metrics on relative canonical bundles are also given.

\section{The general setting}

In this section we will consider a general setup that will subsequently be applied to particular settings in Sections 3 and 4.

We assume we are given a holomorphic submersion $\pi: \mathscr{X} \rightarrow S$ of relative dimension $n$ over a connected base and a relatively ample line bundle $\mathscr{L} \rightarrow \mathscr{L}$. In the absolute case when $S$ is a point we will often use the notation $L \rightarrow X$ for the corresponding ample line bundle. In this latter case we will write $\mathscr{H}_{L}$ for the space of all smooth Hermitian metrics on $L$ with positive curvature form. In the relative case we will denote by $\mathscr{H}_{\mathscr{L} / S}$ the space of all metrics on $\mathscr{L}$ which are fiberwise of positive curvature. We will denote by $c_{1}(L)$ the first Chern class of $L$, normalized so that it lies in $H^{1,1}(X) \cap H^{2}(X, \mathbb{Z})$. To simplify the formulas to be discussed we will also assume that the relative volume of $\mathscr{L}$ is equal to one; that is,

$$
V:=\int_{X} c_{1}(L)^{n} / n !=1
$$

for some (and hence any) fiber $X$. The general formulas may then be obtained by trivial scalings by $V$ at appropriate places. When considering tensor powers of $L$, written as $k L$ in additive notation, we will always assume that $k L$ is very ample (which is true for $k$ sufficiently large).

2A. The weight notation for $\mathscr{H}_{L}$. It will be convenient to use the "weight" representation of a metric $h$ on $L$ : locally, any metric $h$ on $L$ may be represented as $h=e^{-\phi}$, where $h$ is the pointwise norm of a local trivializing section $s$ of $L$. We will call the additive object $\phi$ a "weight" on $L$. One basic feature of this formalism is that even though the functions representing $\phi$ are merely locally defined the normalized curvature form of the metric $h$ may be expressed as

$$
\omega_{\phi}:=d d^{c} \phi:=\frac{i}{2 \pi} \partial \bar{\partial} \phi
$$

which is hence globally well-defined (but it does not imply that $\omega$ is exact!). The normalizations are made so that $\left[\omega_{\phi}\right]=c_{1}(L) \in H^{1,1}(X) \cap H^{2}(X, \mathbb{Z})$. In the absolute setting we will denote by $\mathscr{H}_{L}$ the space of all weights such that $\omega_{\phi}>0$. In other words, the map $\phi \mapsto \omega_{\phi}$ establishes an isomorphism between $\mathscr{H}_{L} / \mathbb{R}$ and the space of Kähler metrics in $c_{1}(L)$. In the relative setting we will denote by $\mathscr{H}_{\mathscr{L} / S}$ the space of all smooth weights on $\mathscr{L}$ such that the restriction to each fiber is of positive curvature.

After fixing a reference weight $\phi_{0}$ in $\mathscr{H}_{L}$ the map $\phi \mapsto u:=\phi-\phi_{0}$ identifies the affine space of all smooth weights on $L$ with the vector space $\mathscr{C}^{\infty}(X)$. Moreover, the subspace $\mathscr{H}_{L}$ of all positively curved smooth weights gets identified with the open convex subspace $\mathscr{H}_{\omega}:=\left\{u: d d^{c} u+\omega_{0}>0\right\}$ 
of $\mathscr{C}^{\infty}(X)$, where $\omega_{0}$ denotes the Kähler form $d d^{c} \phi_{0}$. The $L^{1}$-closure of $\mathscr{H}_{\omega}$ is usually called the space of all $\omega_{0}$-plurisubharmonic functions in the literature [Guedj and Zeriahi 2005]. In fact, all the results in the present paper whose formulation does not use that the given class $\left[\omega_{0}\right]$ is integral are valid in the more general setting when $\mathscr{H}_{L}$ is replaced by $\mathscr{H}_{\omega}$ (with essentially the same proofs). However, since the quantized setting (Section 2D) only makes sense for integral classes we will stick to the weight notation in the following.

2B. The measure $\mu_{\phi}$ and associated functionals on $\mathscr{H}_{L}$. First consider the absolute case when $S$ is a point. In each particular setting studied in Sections 3 and 4 we will assume given a function $\mu$ on $\mathscr{H}_{L}$, $\phi \mapsto \mu(\phi)$ (also denoted by $\mu_{\phi}$ ), taking values in the space of volume forms on $X$, which is exact in the following sense. First observe that we may identify $\mu(\phi)$ with a one-form on the affine space $\mathscr{H}_{L}$ by letting its action on a tangent vector $v \in \mathscr{C}^{\infty}(X)$ at the point $\phi \in \mathscr{H}_{L}$ be defined by

$$
\langle\mu(\phi), v\rangle:=\int_{X} v \mu(\phi) .
$$

The assumption on $\mu(\phi)$ is then simply that this one-form is closed and hence exact; that is, there is a functional $I_{\mu}$ on $\mathscr{H}_{L}$ such that $d I_{\mu}=\mu$ :

$$
\frac{d I_{\mu}\left(\phi_{t}\right)}{d t}=\int_{X} \frac{\partial \phi_{t}}{\partial t} \mu_{\phi_{t}}
$$

for any path $\phi_{t}$ in $\mathscr{H}_{L}$. The functional is determined up to a constant which will be fixed in each particular setting to be studied. We will also assume that for any fixed $v \in \mathscr{C}^{\infty}(X)$ the functional $\phi \mapsto\langle\mu(\phi), v\rangle$ is continuous with respect to the $L^{\infty}$-topology on $\mathscr{H}_{L}$.

Two particular examples of such exact one-forms and their antiderivatives that will be used repeatedly are as follows:

- The Monge-Ampère measure $\phi \mapsto\left(d d^{c} \phi\right)^{n} / n !:=\operatorname{MA}(\phi)$. Its antiderivative [Mabuchi 1986] will be denoted by $\mathscr{E}(\phi)$, normalized so that $\mathscr{E}\left(\phi_{0}\right)=0$ for a fixed reference weight $\phi_{0}$ in $\mathscr{H}_{L}$. Integrating along line segments in $\mathscr{H}_{L}$ gives an explicit expression for $\mathscr{E}$, but it will not be used here.

- $\phi \mapsto \mu_{0}$ for a volume form $\mu_{0}$ on $X$, fixed once and for all with $I_{\mu_{0}}(\phi):=\int_{X}\left(\phi-\phi_{0}\right) \mu_{0}$. Since we have already fixed a reference weight $\phi_{0}$ it will be convenient to take $\mu_{0}:=\left(d d^{c} \phi_{0}\right)^{n} / n !$.

Given $\mu=\mu(\phi)$ we define the associated functional

$$
\mathscr{F}_{\mu}:=\mathscr{E}-I_{\mu} .
$$

By construction its critical points in $\mathscr{H}_{L}$ are precisely the solutions to the Monge-Ampère equation

$$
\left(d d^{c} \phi\right)^{n} / n !=\mu(\phi) .
$$

We will say that $\mu(\phi)$ is normalized if it is a probability measure for all $\phi$. Equivalently, this means that $I_{\mu}$ is equivariant under scalings; that is, $I_{\mu}(\phi+c)=I_{\mu}(\phi)+c$ which in turn is equivalent to $\mathscr{F}_{\mu}$ being invariant under scalings. 
In the relative setting we assume that $\mu_{S}(\phi)$ is a smooth family of measures on the fibers $\mathscr{X}_{s}$ as above, parametrized by $s \in S$.

Properness and coercivity. We first recall the definition of the well-known $J$-functional, defined with respect to a fixed reference weight $\phi_{0}$ (see [Berman et al. 2009] for a general setting and references). It is the natural higher-dimensional generalization of the (squared) Dirichlet norm on a Riemann surface and it will play the role of an exhaustion function of $\mathscr{H}_{L} / \mathbb{R}$ (but without specifying any topology!). In our notation $J$ is simply given by the scale-invariant function

$$
J=-\mathscr{F} \mu_{0} .
$$

We will then say that a functional $\mathscr{G}$ is proper if

$$
J \rightarrow \infty \Longrightarrow \mathscr{G} \rightarrow \infty
$$

and coercive if there exists $\delta>0$ and $C_{\delta}$ such that

$$
\varphi \geq \delta J-C_{\delta} .
$$

Note that $\delta$ may be taken arbitrarily small at the expense of increasing $C_{\delta}$. In many geometric applications properness (and coercivity) of suitable functionals can be thought as analytic versions of algebro-geometric stability (compare Remark 4.2).

2C. The relative Kähler-Ricci flow with respect to $\mu_{\phi}$. Given an initial weight $\phi_{0} \in \mathscr{H}_{\mathscr{L} / S}$ the relative Kähler-Ricci flow in $\mathscr{H}_{\mathscr{L} / S}$ is defined by the fiberwise parabolic Monge-Ampère equation

$$
\frac{\partial \phi_{t}}{\partial t}=\log \frac{\left(d d^{c} \phi_{t}\right)^{n} / n !}{\mu\left(\phi_{t}\right)}
$$

for $\phi_{t}$ smooth over $\mathscr{X} \times[0, T]$, where $T \geq 0$. We will make the following assumptions on the flow which will all be satisfied in the particular settings studied in Sections 3 and 4.

Analytical assumptions on the flow.

- Existence: The flow exists and is smooth over $\mathscr{X} \times[0, \infty[$.

- Uniqueness: Any fixed point in $\mathscr{H}_{L}$ of the flow is unique $\bmod \mathbb{R}$.

- Stability: For any $l>0$ and $M>0$ there is a constant $B_{l, M}$ only depending on the upper bound on the $\mathscr{C}^{l}$-norm of the initial weight $\phi_{0}$ (with respect to a fixed reference weight) and a lower bound on the absolute value of $d d^{c} \phi_{0}$ such that

$$
\left\|\phi_{t}-\phi_{0}\right\|_{\mathscr{C}^{l}(X \times[0, M])} \leq B_{l, M}
$$

(locally uniformly with respect to $s$ in the relative setting).

It follows immediately that $\phi$ is fixed under the flow if and only if it solves the Monge-Ampère equation $(2-2)$. Note that since we have assumed that $\operatorname{Vol}(L)=1$, a necessary condition to be stationary is that 
$\int_{X} \mu_{\phi}=1$. For any solution $\phi_{t}$ and fixed fiber $X=\mathscr{X}_{s}$ the Kähler metrics $\omega_{t}$ on $X$ obtained as the restricted curvature forms of $\phi_{t}$ hence evolve according to

$$
\frac{\partial \omega_{t}}{\partial t}=-\operatorname{Ric} \omega_{t}-\eta_{\mu}
$$

where Ric $\omega_{t}$ in the Ricci curvature of the Kähler metric $\omega_{t}$ and $\eta_{\mu}=d d^{c} \log \mu(\phi)$.

Thanks to the following simple lemma the Kähler-Ricci flow is "gradient-like" for the functional $\mathscr{F}_{\mu}$. For the Fano case, see [Chen and Tian 2002].

Lemma 2.1. The functional $\mathscr{F}_{\mu}$ is increasing along the Kähler-Ricci flow on $\mathscr{H}_{L}$ (defined with respect to $\left.\mu_{\phi}\right)$. Moreover, it is strictly increasing at $\phi_{t}$ unless $\phi_{t}$ is stationary.

Proof. Differentiating along the flow gives

$$
\frac{d \mathscr{F}\left(\phi_{t}\right)}{d t}=\int_{X} \log \frac{\operatorname{MA}\left(\phi_{t}\right)}{\mu\left(\phi_{t}\right)}\left(\operatorname{MA}\left(\phi_{t}\right)-\mu\left(\phi_{t}\right)\right)=\int_{X} \log \frac{\operatorname{MA}\left(\phi_{t}\right)}{\mu\left(\phi_{t}\right)}\left(\frac{\operatorname{MA}\left(\phi_{t}\right)}{\mu\left(\phi_{t}\right)}-1\right) \mu\left(\phi_{t}\right) \geq 0
$$

where the last inequality follows since both factors in the last integrand clearly have the same sign.

If, moreover, $\mu(\phi)$ is normalized then both terms appearing in the definition of $\mathscr{F}_{\mu}$ are monotone:

Lemma 2.2. Assume that $\mu(\phi)$ is normalized. Then the functionals $-I_{\mu}$ and $\mathscr{E}$ are both increasing along the Kähler-Ricci flow on $\mathscr{H}_{L}$ with respect to $\mu(\phi)$. Moreover, they are strictly increasing at $\phi_{t}$ unless $\phi_{t}$ is stationary.

Proof. Differentiating along the flow gives

$$
-\frac{d I\left(\phi_{t}\right)}{d t}=-\int_{X} \log \frac{\mathrm{MA}\left(\phi_{t}\right)}{\mu\left(\phi_{t}\right)} \mu\left(\phi_{t}\right) \geq 0
$$

using Jensen's inequality applied to the concave function $f(t)=\log t$ on $\mathbb{R}_{+}$in the last step (recall that $\operatorname{MA}\left(\phi_{t}\right), \mu\left(\phi_{t}\right)$ are both probability measures). Similarly,

$$
\frac{d \mathscr{E}\left(\phi_{t}\right)}{d t}=\int_{X} \log \frac{\operatorname{MA}\left(\phi_{t}\right)}{\mu\left(\phi_{t}\right)} \operatorname{MA}\left(\phi_{t}\right)=-\int_{X} \log \frac{\mu\left(\phi_{t}\right)}{\operatorname{MA}\left(\phi_{t}\right)} \operatorname{MA}\left(\phi_{t}\right) \geq 0,
$$

again using Jensen's inequality, but with the roles of $\operatorname{MA}\left(\phi_{t}\right), \mu\left(\phi_{t}\right)$ reversed. The statement about strict monotonicity also follows from Jensen's inequality since $f(t)=\log t$ is strictly concave.

From the previous lemma we deduce the following compactness property of the flow.

Lemma 2.3. Assume that $\mu(\phi)$ is normalized and that the associated functional-F $\mu$ is coercive. Then there is a constant $C$ such that $J\left(\phi_{t}\right) \leq C$ and $\int\left|\phi_{t}-\phi_{0}\right| \mu_{0} \leq C$ along the Kähler-Ricci flow for $\phi_{t}$ (with respect to $\mu(\phi)$ ).

Proof. Combining the monotonicity of $\mathscr{F}_{\mu}$ and the assumption that $\mathscr{F}_{\mu}$ be coercive (and in particular proper) immediately gives the first inequality $J\left(\phi_{t}\right) \leq C$. Next, by the definition of coercivity there are $\delta \in] 0,1\left[\right.$ and $C_{\delta}>0$ such that $I_{\mu}-\mathscr{E} \geq \delta I_{\mu_{0}}-\delta \mathscr{E}-C_{\delta}$; that is,

$$
\delta I_{\mu_{0}} \leq(-1+\delta) \mathscr{E}+I_{\mu}+C_{\delta}
$$


along the flow. Since by the previous lemma $-\mathscr{E}$ and $I_{\mu}$ are both bounded from above along the flow it follows that there is a constant $A$ such that $I_{\mu_{0}} \leq A$ along the flow. Finally, by basic pluripotential theory the set $\left\{\phi \in \mathscr{H}_{L}: J(\phi) \leq C, I_{\mu_{0}}(\phi) \leq C\right\}$ is relatively compact in the $L^{1}$-topology [Berman et al. 2009]. This proves the last inequality in the statement of the lemma.

The next proposition shows that, under suitable assumptions, the Kähler-Ricci flow with respect to a normalized measure $\mu_{\phi}$ converges on the level of weights precisely when it converges on the level of Kähler metrics. In Sections 3 and 4 the proposition will be applied to the usual geometric Kähler-Ricci flows, where the convergence is already known to hold on the level of Kähler metrics. To simplify the notation we will only state the result in the absolute case, the extension to the relative case being immediate.

Proposition 2.4. Assume that $\mu(\phi)$ is normalized and that the associated functional $-\mathscr{F}_{\mu}$ is coercive. Let $\phi_{t}$ evolve according to the Kähler-Ricci flow defined with respect to $\mu_{\phi}$ and write $\omega_{t}=d d^{c} \phi_{t}$. Then the following three statements are all equivalent:

- The sequence of Kähler metrics $\omega_{t}$ is relatively compact in the $\mathscr{C}^{\infty}$-topology on $X$; that is, for any positive integer $l$ the sequence $\omega_{t}$ is uniformly bounded in the $\mathscr{C}^{l}$-norm on $X$.

- The weights converge: $\phi_{t} \rightarrow \phi_{\infty} \in \mathscr{H}_{L}$ in the $\mathscr{C}^{\infty}$-topology on $X$ as $t \rightarrow \infty$.

- The Kähler metrics $\omega_{t} \rightarrow \omega_{\infty}$ in the $\mathscr{C}^{\infty}$-topology on $X$, where $\omega_{\infty}$ is a Kähler form.

Proof. Assume that the first point of the proposition holds. Then it is a basic fact that the sequence of normalized weights $\tilde{\phi}_{t}:=\phi_{t}-C_{t}$, where $C_{t}:=I_{\mu_{0}}\left(\phi_{t}\right)$, is relatively compact in the $\mathscr{C}^{\infty}$-topology on $X$ and converges to $\tilde{\phi}_{\infty} \in \mathscr{H}_{L}$ (as is seen by inverting the associated Laplacians). By the previous lemma $\left|C_{t}\right| \leq D$ for some positive constant $D$ and hence $\left\{\phi_{t}\right\}$ is also relatively compact in the $\mathscr{C}^{\infty}$-topology on $X$.

In the rest of the argument we will use the $\mathscr{C}^{l}$-topology on $\mathscr{H}_{L}$ for $l$ a large fixed integer. Let $\mathscr{K}:=\overline{\left\{\phi_{t}\right\}}$ be the closure of $\left\{\phi_{t}\right\}$ which is relatively compact in $\mathscr{H}_{L}$ by the previous argument. Denote by $\psi_{0}$ an accumulation point in $\mathscr{K}$ :

$$
\lim _{j} \phi_{t_{j}}=\psi_{0} .
$$

By continuity of the "time $s$ flow map" (which follows immediately from the stability assumption on the flow) and the semigroup structure of the flow we deduce that

$$
\lim _{j} \phi_{t_{j}+s}=\psi_{s}
$$

for any fixed $s>0$. In other words, $\mathscr{K}$ is in fact compact and invariant under the "time $s$ flow map". Note also that by monotonicity

$$
\lim _{t} \mathscr{E}\left(\phi_{t}\right)=\mathscr{E}\left(\psi_{0}\right)=\sup _{\mathscr{K}} \mathscr{E} .
$$

Assume now to get a contradiction that $\psi_{s} \neq \psi_{0}$. By the strict monotonicity in Lemma 3.8 we have that $\mathscr{E}\left(\psi_{s}\right)>\mathscr{E}\left(\psi_{0}\right)$, contradicting (2-6) (since $\psi_{s} \in \mathscr{K}$ as explained above). Hence, $\psi_{0}$ is a fixed point of the 
flow and hence, by the uniqueness assumption on the flow, it is determined up to an additive constant. This means that for any two limit points $\psi_{0}$ and $\psi_{0}^{\prime}$ of the flow there is a constant $C$ such that

$$
\psi_{0}-\psi_{0}^{\prime}=C
$$

But as explained above $\mathscr{E}\left(\psi_{0}\right)=\mathscr{E}\left(\psi_{0}^{\prime}\right)$ and hence, by the scaling equivariance of $\mathscr{E}$, it follows that $C=0$. All in all this means that we have shown that the flow $\phi_{t}$ converges, in the $\mathscr{C}^{\infty}$-topology on $X$, to a limit $\phi_{\infty}$ in $\mathscr{H}_{L}$, that is, that the second point of the proposition holds. The rest of the implications are trivial.

Remark 2.5. The coercivity is used to make sure that the compactness property of the flow $\phi_{t}$ holds without normalizing $\phi_{t}$ (say, by subtracting $I_{\mu_{0}}\left(\phi_{t}\right)$ ). If one only assumes properness then the same proof shows that the statement still holds upon replacing $\phi_{t}$ by $\phi_{t}-I_{\mu_{0}}\left(\phi_{t}\right)$ (which, of course, does not effect the curvature forms). The same remark applies to Proposition 2.9 below.

2D. Quantization: The Bergman iteration on $\mathscr{H}_{L}$. Proceeding fiberwise it will be enough to consider the absolute case when $S$ is a point and we are given an ample line bundle $L \rightarrow X$. For any positive integer $k$ such that $k L$ is very ample the quantization at level $k$ of the space $\mathscr{H}_{L}$ is defined as the space $\mathscr{H}^{(k)}$ of all Hermitian metrics on the $N_{k}$-dimensional complex vector space $H^{0}(X, k L)$. Hence, $\mathscr{H}^{(k)}$ may be identified with the symmetric space $\operatorname{GL}\left(N_{k}, \mathbb{C}\right) / U\left(N_{k}\right)$. In the relative setting $\mathscr{H}^{(k)}$ is replaced by the space of all Hermitian metrics on the rank- $N_{k}$ vector bundle $\pi_{*}(k \mathscr{L})$ over the base $S$ (compare the discussion at the bottom of page 156).

Fix a volume form $\mu_{\phi}$ on $X$ depending on $\phi$ as above. Then any given $\phi \in \mathscr{H}_{L}$ induces a Hermitian metric $\operatorname{Hilb}^{(k)}(\phi)$ defined by

$$
\operatorname{Hilb}^{(k)}(\phi)(f, f):=\int_{X}|f|^{2} e^{-k \phi} d \mu_{\phi},
$$

giving a map

$$
\operatorname{Hilb}^{(k)}: \mathscr{H}_{L} \rightarrow \mathscr{H}^{(k)} .
$$

There is also a natural injective map (independent of $\mu_{\phi}$ ) in the reverse direction, called the (scaled) Fubini-Study map $\mathrm{FS}^{(k)}$ :

$$
\mathrm{FS}^{(k)}(H):=\log \left(\frac{1}{N_{k}} \sum_{i=1}^{N_{k}}\left|f_{i}^{H}\right|^{2}\right)
$$

where $\left(f_{i}^{H}\right)$ is any basis in $H^{0}(X, k L)$ that is orthonormal with respect to $H$.

Donaldson's iteration (with respect to $\mu_{\phi}$ ) on the space $\mathscr{H}^{(k)}$ is then obtained by iterating the composed map

$$
T^{(k)}:=\operatorname{Hilb}^{(k)} \circ \mathrm{FS}^{(k)}: \mathscr{H}^{(k)} \rightarrow \mathscr{H}^{(k)},
$$

and its fixed points are called balanced metrics at level $k$ (with respect to $\mu$ ).

In order to facilitate the comparison with the Kähler-Ricci flow it will be convenient to consider the (essentially equivalent) iteration on the space $\mathscr{H}_{L}$ obtained by iterating the map $\mathrm{FS}^{(k)} \circ \mathrm{Hilb}^{(k)}$. This 
latter iteration will be called the Bergman iteration at level $k$ (with respect to $\mu_{\phi}$ ) and we will denote the $m$-th iterate by $\phi_{m}^{(k)}$ and call the parameter $m$ discrete time. Hence, the iteration immediately enters the finite-dimensional submanifold $\operatorname{FS}\left(\mathscr{H}^{(k)}\right) \subset \mathscr{H}_{L}$ of Bergman metrics at level $k$ and stays there forever. By the very definition of the Bergman iteration it may be written as the difference equation

$$
\phi_{m+1}^{(k)}-\phi_{m}^{(k)}=\frac{1}{k} \log \rho^{(k)}\left(\phi_{m}^{(k)}\right),
$$

where $\rho^{(k)}(\phi)$ is the Bergman function at level $k$ associated to $\left(\mu_{\phi}, \phi\right)$; that is,

$$
\rho^{(k)}(\phi)=\frac{1}{N_{k}} \sum_{i=1}\left|f_{i}\right|^{2} e^{-k \phi},
$$

where $f_{i}$ is an orthonormal basis with respect to the Hermitian metric $\operatorname{Hilb}^{(k)}(\mu, \phi)$. Note that the Bergman measure $\rho^{(k)}(\phi) \mu_{\phi}$ is a probability measure on $X$ and independent of the choice of orthonormal bases. It plays the role of the Monge-Ampère measure in the quantized setting.

It will also be convenient, following [Donaldson 2009], to study functionals defined directly on the space $\mathscr{H}^{(k)}$. Fixing the reference metric $H_{0}^{(k)}:=\operatorname{Hilb}^{(k)}\left(\phi_{0}\right) \in \mathscr{H}^{(k)}$ we may identify $\mathscr{H}^{(k)}$ with the space of all rank $N_{k}$ Hermitian matrices. We define

$$
\mathscr{F}_{\mu}^{(k)}(H):=-\frac{1}{N_{k} k} \log \operatorname{det} H-I_{\mu} \circ \mathrm{FS}^{(k)}(H),
$$

whose critical points in $\mathscr{H}^{(k)}$ are precisely the balanced metrics (with respect to $\mu_{\phi}$ ); this is proved exactly as in the particular cases considered in [Donaldson 2005; Berman et al. 2009]. We will also consider the following functional on $\mathscr{H}_{L}$ :

$$
\mathscr{L}^{(k)}(\phi):=-\frac{1}{N_{k} k} \log \operatorname{det} \operatorname{Hilb}^{(k)}\left(\mu_{\phi}, \phi\right),
$$

normalized so that $\mathscr{L}^{(k)}(\phi+c)=\mathscr{L}^{(k)}(\phi)+c$. Equivalently, we could have defined $\mathscr{L}^{(k)}$ as the antiderivative of the one-form on $\mathscr{H}_{L}$ defined by integration against the Bergman measure $\rho^{(k)}(\phi) \mu_{\phi}$.

Monotonicity. The following monotonicity properties were shown in [Donaldson 2009] in the particular setting considered there (where $\mu_{\phi}$ is independent of $\phi$ ). See also [Donaldson 2005] for the setting when $\mu(\phi)=\operatorname{MA}(\phi)$ (compare Section $4 \mathrm{~F}$ ). The main new observation here is that concavity of $I_{\mu}$ implies monotonicity.

Lemma 2.6. Assume that $\mu_{\phi}$ is normalized. Then the following monotonicity with respect to the discrete time $m$ holds along the Bergman iteration $\phi_{m}^{(k)}$ on $\mathscr{H}_{L}$ (defined with respect to $\left.\mu_{\phi}\right)$ :

- The functional $\mathscr{L}^{(k)}$ is increasing along the Bergman iteration and strictly increasing at $\phi_{m}^{(k)}$ unless $\phi_{m}^{(k)}$ is stationary. Equivalently, the functional-log det is strictly increasing along the Donaldson iteration in $\mathscr{H}^{(k)}$ away from balanced metrics. 
- If $I_{\mu}$ is concave on the space $\mathscr{H}_{L}$ with respect to the affine structure then it is decreasing along the iteration and strictly decreasing at $\phi_{m}^{(k)}$ unless $\phi_{m}^{(k)}$ is stationary. Equivalently, the functional $I_{\mu} \circ \mathrm{FS}^{(k)}$ is strictly decreasing along the Donaldson iteration in $\mathcal{H}^{(k)}$ away from balanced metrics.

Proof. The proof of the first point is essentially the same as in Donaldson's setting [2009], but for completeness we repeat it here. By definition

$$
\mathscr{L}^{(k)}\left(\phi_{m+1}\right)-\mathscr{L}^{(k)}\left(\phi_{m}\right)=-\frac{1}{N_{k} k} \log \frac{\operatorname{det} \operatorname{Hilb}^{(k)}\left(\phi_{m+1}\right)}{\operatorname{det} \operatorname{Hilb}^{(k)}\left(\phi_{m}\right)} .
$$

By the concavity of log and Jensen's inequality we hence get

$$
\mathscr{L}^{(k)}\left(\phi_{m+1}\right)-\mathscr{L}^{(k)}\left(\phi_{m}\right) \geq-\frac{1}{k} \log \frac{1}{N_{k}} \sum_{i=1}^{N_{k}}\left\|f_{i}\right\|_{T\left(\mathrm{Hilb}^{(k)}\left(\phi_{m}\right)\right)}^{2},
$$

where $f_{i}$ is an orthonormal basis with respect to the Hermitian metric $\operatorname{Hilb}^{(k)}\left(\phi_{m}\right)$ and where by definition $T\left(\operatorname{Hilb}^{(k)}\left(\phi_{m}\right)\right)=\operatorname{Hilb}^{(k)}\left(\mathrm{FS}\left(\operatorname{Hilb}^{(k)}\left(\phi_{m}\right)\right)\right)$. Writing out the norms explicitly shows that the right-hand side above may be written as

$$
-\frac{1}{k} \log \left(\frac{1}{N_{k}}\left(\sum_{i=1}^{N_{k}}\left|f_{i}\right|^{2} / \sum_{i=1}\left|f_{i}\right|^{2}\right) \mu_{\mathrm{FS}\left(\operatorname{Hilb}^{(k)}\left(\phi_{m}\right)\right)}\right)=-\frac{1}{k} \log (1)=0,
$$

using that $\mu_{\phi}$ is normalized. This proves the first point.

To prove the second point we use that $I_{\mu}$ is assumed concave and that, by definition, $\mu_{\phi}=d I_{\mu}$ as a differential, to get

$$
\begin{aligned}
I_{\mu}\left(\phi_{m+1}^{(k)}\right)-I_{\mu}\left(\phi_{m}^{(k)}\right) & \leq \int\left(\phi_{m+1}^{(k)}-\phi_{m}^{(k)}\right) \mu_{\phi_{m}^{(k)}}=\frac{1}{k} \int \log \rho^{(k)}\left(\phi_{m}^{(k)}\right) \mu_{\phi_{m}^{(k)}} \\
& \leq \frac{1}{k} \log \int \rho^{(k)}\left(\phi_{m}^{(k)}\right) \mu_{\phi_{m}^{(k)}}=0,
\end{aligned}
$$

using the definition of the iteration and Jensen's inequality in the last step (and the fact that $\rho^{(k)}(\phi) \mu_{\phi}$ and $\mu_{\phi}$ are both probability measures). This proves the monotonicity of $I_{\mu}$. The statement about strict monotonicity follows immediately from the fact that $\log t$ is strictly concave.

Properness and coercivity. Properness and coercivity of functionals on $\mathscr{H e}^{(k)}$ are defined as in Section 2C, but with the functional $J$ replaced by its quantized version on the space $\mathscr{H}^{(k)}$ :

$$
J^{(k)}(H):=-\mathscr{F}_{\mu_{0}}^{(k)}:=I_{\mu_{0}} \circ \mathrm{FS}^{(k)}+\frac{1}{k N_{k}} \log \operatorname{det} H .
$$

The content of the following lemma is essentially contained in the proof of Proposition 3 in [Donaldson 2009]. We will fix a metric $H_{0} \in \mathscr{H}^{(k)}$. For any given $H_{0}$-orthonormal basis $\left(f_{i}\right)$ we can then identify a Hermitian metric $H$ with a matrix and we will denote by $H_{\lambda}$ the diagonal matrix with entries $e^{-\lambda_{i}}$ on the diagonal. 


\section{Lemma 2.7.}

- For $\lambda \in \mathbb{C}^{N_{k}}$ let $\phi_{\lambda}=\mathrm{FS}^{(k)}\left(H_{\lambda}\right):=\frac{1}{k} \log \left(\frac{1}{N_{k}} \sum_{i} e^{k \lambda_{i}}\left|f_{i}\right|^{2}\right)$. There $i s$ a constant $C$ such that

$$
\max _{i} \lambda_{i} \leq I_{\mu_{0}}\left(\phi_{\lambda}\right)+C \text {. }
$$

- The functional $J^{(k)}$ is an exhaustion function on $\mathscr{H}^{(k)} / \mathbb{R}^{*}$ with respect to its usual topology.

- In particular, the set of all $H \in \mathscr{H}^{(k)}$ such that

$$
-\log \operatorname{det} H \geq-C, \quad\left(I_{\mu_{0}} \circ \mathrm{FS}\right)(H) \leq C
$$

is relatively compact.

Proof. For the benefit of the reader we repeat Donaldson's simple proof: let $i_{\max }$ be an index such that $\max _{i} \lambda_{i}=\lambda_{i_{\max }}$. Clearly,

$$
\max _{i} \lambda_{i}+\frac{1}{k} \log \left(\frac{1}{N_{k}}\left|f_{i_{\max }}\right|^{2}\right) \leq \phi_{\lambda} \leq \max _{i} \lambda_{i}+\frac{1}{k} \log \left(\frac{1}{N_{k}} \sum_{i}\left|f_{i}\right|^{2}\right),
$$

and hence integrating over $X$ and using the first inequality above gives

$$
\max _{i} \lambda_{i}+\int_{X}\left(\log \left(\left|f_{i_{\max }}\right|^{2}\right)-\phi_{0}\right) d \mu_{0} \leq I_{\mu_{0}}\left(\phi_{\lambda}\right),
$$

which proves the lemma since it is well-known that $I_{\mu_{0}}(\psi)>-\infty$ for any psh (plurisubharmonic) weight $\psi$ if $\mu_{0}$ is a smooth volume form (as follows from the local fact that any psh function is in $L^{1}$ ) and in particular $-C:=I_{\mu}\left(\log \left(\left|f_{i_{\text {max }}}\right|^{2}\right)\right)>-\infty$. This proves the first point. As for the second and third one we first note that any Hermitian metric $H$ can be represented by a diagonal matrix (which we write in the form $H_{\lambda}$ ) after perhaps changing the basis $\left(f_{i}\right)$ above. Moreover, by the compactness of $U(N)$ the constant $C$ in the previous point can be taken to be independent of the base $\left(f_{i}\right)$.

Next, it will be enough to prove the last point of the lemma (the second point then follows since we may by scaling invariance assume that $\operatorname{det}\left(H_{\lambda}\right)=1$ ). We may assume that $\inf _{i} \lambda_{i}=\lambda_{0}$ and since, by assumption,

$$
-\log \operatorname{det} H=\sum_{i} \lambda_{i} \geq-C
$$

we get

$$
-\inf _{i} \lambda_{i} \leq C+\sum_{i \neq 0} \lambda_{i} \leq C+(N-1) \max _{i} \lambda_{i}
$$

By the assumption $\left(I_{\mu_{0}} \circ \mathrm{FS}\right)(H) \leq C$ and the first point of the lemma the right-hand side above is bounded from above and hence we conclude that so is $-\inf _{i} \lambda_{i}$. All in all this means that $\max _{i}\left|\lambda_{i}\right|$ is uniformly bounded from above by a constant; that is, $H$ stays in a relatively compact subset of $\mathscr{H}^{(k)}$.

Remark 2.8. The proof of the previous lemma shows that the conclusion of the lemma remains valid for any choice of a fixed reference weight $\phi_{0}$ and probability measure $\mu_{0}$ (which are used in the definition of $\left.J^{(k)}\right)$ such that $\int_{X} \log \left(|f|-\phi_{0}\right) \mu_{0}$ is finite for any section $f \in H^{0}(X, k L)$. 


\section{Criteria for convergence in the large time limit.}

Proposition 2.9. Assume that $\mu_{\phi}$ is normalized, that $I_{\mu}$ is decreasing along the Bergman iteration, that $\mathscr{F}_{\mu}^{(k)}$ is coercive and that there is at most one balanced metric (modulo scaling). Then, for any given positive integer $k$ the following holds: in the large time limit, that is, when $m \rightarrow \infty$, the weights $\phi_{m}^{(k)} \rightarrow \phi_{\infty}^{(k)}$ in the $\mathscr{C}^{\infty}$-topology on $X$. Moreover, in the relative setting the convergence is uniform with respect to the base parameter $s$.

Proof. (a) Uniform convergence. We equip FS( $\left.\mathscr{H}^{(k)}\right)$, that is, the space of all Bergman weights at level $k$, with the topology induced by the sup norm. It is not hard to see that this is the same topology as the one induced from the finite-dimensional symmetric space $\mathscr{H}^{(k)}=\operatorname{GL}\left(N_{k}, \mathbb{C}\right) / U\left(N_{k}\right)$ with its usual Riemannian metric, or with respect to the operator norm on $\operatorname{GL}\left(N_{k}, \mathbb{C}\right)$. Hence, it will be enough to prove the convergence of Donaldson's iteration in $\mathscr{H}^{(k)}$.

Since $\mu_{\phi}$ is assumed normalized, Lemma 2.6 shows that $-\log \operatorname{det} H$ is uniformly bounded from below along the Donaldson iteration in $\mathscr{H}^{(k)}$. Moreover, by assumption $I_{\mu_{\phi}} \circ \mathrm{FS}^{(k)}$ is uniformly bounded from above along the Donaldson iteration. Hence, just as in the proof of Lemma 2.3 it follows from the coercivity assumption that $I_{\mu_{0}} \circ \mathrm{FS}^{(k)}$ is also uniformly bounded from above along the Donaldson iteration. But then it follows from Lemma 2.7 that the iteration $H_{m}^{(k)}$ stays in a compact subset of $\mathcal{H}^{(k)}$.

Now let $\left.\mathscr{K}:=\overline{\left\{H_{m}^{(k)}\right.}\right\}$ be the closure of the orbit of $T^{(k)}$ which is relatively compact in $\mathscr{H}^{(k)}$ by the previous argument. Denote by $G$ an accumulation point

$$
\lim _{j} H_{m_{j}}^{(k)}=G
$$

in $\mathscr{H}^{(k)}$. By the continuity of $H \mapsto T^{(k)}(H)$ on $\mathscr{H}^{(k)}$ we deduce that

$$
\lim _{j} T^{(k)}\left(H_{m_{j}}^{(k)}\right)=T^{(k)}(G) .
$$

In other words, $\mathscr{K}$ is in fact compact and invariant under $T^{(k)}$. Note also that by monotonicity

$$
\lim _{j}\left(-\log \operatorname{det} H_{m_{j}}^{(k)}\right)=-\log \operatorname{det} G=\sup _{\mathscr{K}}(-\log \operatorname{det}) .
$$

Assume now to get a contradiction that $T^{(k)}(G) \neq G$. By the strict monotonicity in Lemma 3.8 we have $\log \operatorname{det}\left(T^{(k)} G\right)>\log \operatorname{det} G$, contradicting (2-6) (since $T^{(k)}(G) \in \mathscr{K}$ ). All in all this means that we have shown that the subsequence $\left(H_{m_{j}}^{(k)}\right)$ of Donaldson iterations converges to a fixed point, that is, a balanced metric. By the assumption on uniqueness up to scaling it follows, again using monotonicity (just like in the proof of Proposition 2.4), that all accumulation points coincide; that is, the iteration converges.

(b) Higher order convergence. To simplify the notation we set $k=1$ and write $\phi_{m}^{(k)}=\phi_{m}$. First note that the $L^{\infty}$-estimate above is uniform over $S$, as follows by combining the monotonicity of the functionals with the uniform boundedness of the initial weight $\phi_{0}$. By the uniform convergence of $\phi_{m}$ it will hence be enough to prove that

$$
\left\|\partial_{X}^{\alpha}\left(h_{0} / h_{m+1}\right)\right\|_{L^{\infty}(X)} \leq C_{\alpha}\left\|\left(h_{m} / h_{0}\right)\right\|_{L^{\infty}(X)}
$$


where $h_{m}=e^{-\phi_{m}}$ and $\partial_{X}^{\alpha}$ denotes a real linear differential operator on $X$ of order $\alpha$ (note that while $h_{m}$ globally corresponds to a metric on $L$ the quotient $h_{0} / h_{m+1}$ defines a global function on $X$ ). Accepting this estimate for the moment the uniform convergence of $\left(h_{m}\right)$ hence gives that $\left\|\partial_{X}^{\alpha}\left(h_{0} / h_{m}\right)\right\|_{L^{\infty}(X)}$ is uniformly bounded in $m$ and since $h_{m} / h_{0} \rightarrow h_{\infty} / h_{0}$ it then follows that $\left\|\partial_{X}^{\alpha}\left(\phi_{m}-\phi_{0}\right)\right\|_{L^{\infty}(X)}$ is also uniformly bounded in $m$. Hence, standard compactness arguments show the $\mathscr{C}^{\infty}$-convergence of $\left(\phi_{m}\right)$.

Finally, the estimate (2-9) is a consequence of the following quasiexplicit integral formula for the Bergman function familiar from the theory of determinantal random point processes (see [Berman 2008] and references therein):

$\rho(\phi)(x)=\int_{y \in X^{N-1}} f(x, y) e^{-\left(\phi-\phi_{0}\right)(x)} e^{-\left(\phi-\phi_{0}\right)(y)} d \mu_{\phi}(y)^{\otimes N-1} / Z_{\phi}, \quad Z_{\phi}:=\int_{X^{N}} f_{0} e^{-\left(\phi-\phi_{0}\right)} d \mu_{\phi}^{\otimes N}$ where $f\left(x_{1}, x_{2}, \ldots, x_{N}\right)=\left|\operatorname{det}_{1 \leq i, j \leq N}\left(f_{i}\left(x_{i}\right)\right)_{i, j}\right|^{2} e^{-\phi_{0}\left(x_{1}\right)} \cdots e^{-\phi_{0}\left(x_{N}\right)}$ and $\left(f_{i}\right)$ is any given orthonormal base with respect to the Hermitian metric $\operatorname{Hilb}^{(1)}\left(\phi_{0}\right)$ on $H^{0}(X, L)$ (note that $Z_{\phi}$ appears as the normalizing constant). We have used the notation $\phi\left(x, \ldots, x_{m}\right)=\phi\left(x_{1}\right)+\cdots+\phi\left(x_{m}\right)$. In particular,

$$
\left(h_{0} / h_{m+1}\right)(x)=\int_{y \in X^{N-1}} f(x, y) e^{-\left(\phi_{m}-\phi_{0}\right)(y)} d \mu_{\phi}(y)^{\otimes N-1} / Z_{\phi_{m}}
$$

and hence differentiating with respect to $x$ by applying $\partial_{X}^{\alpha}$ gives

$$
\left|\partial_{X}^{\alpha}\left(h_{0} / h_{m+1}\right)(x)\right|=\left|\int\left(\partial_{X}^{\alpha} f(x, y)\right) e^{-\left(\phi-\phi_{0}\right)(y)} d \mu_{\phi}(y)^{\otimes N-1} / Z_{\phi}\right| \leq \frac{A_{\alpha}}{Z_{\phi_{m}}}\left\|e^{-\left(\phi_{m}-\phi_{0}\right)}\right\|_{L^{\infty}(X)},
$$

where $A_{\alpha}$ is a constant independent of $m$. Since, by the uniform convergence of $\phi_{m}$, we have $Z_{\phi_{m}}>C>0$ for some positive constant $C$, this concludes the proof of the estimate (2-9).

The following basic lemma gives a natural criterion for the assumptions (apart from the monotonicity of $\left.I_{\mu}\right)$ in the previous theorem to be satisfied.

Lemma 2.10. Suppose that $\varphi$ is a functional on $\mathcal{H e}^{(k)}$ which is geodesically strictly convex with respect to the symmetric Riemann structure and strictly convex modulo scaling. Then $G$ has at most one critical point (modulo scaling). Moreover, if it has some critical point then $G$ is coercive.

Proof. Uniqueness follows immediately from strict convexity and hence we turn to the proof of coercivity. By a simple compactness argument it will be clear that, after fixing a reference metric $H_{0} \in \mathscr{H}^{(k)}$, which we take to be a critical point of $\mathscr{G}$, it is enough to prove coercivity along any fixed geodesic passing through $H_{0}$. To this end let $H_{t}$ be a geodesic in $\mathscr{H}^{(k)}$ starting at $H_{0}$, that is, the orbit of the action of a one-parameter subgroup of GL $\left(N_{k}\right)$. In the notation of Lemma 2.7 this means that $H_{t}=H_{t \lambda}$ for $\lambda \in \mathbb{C}^{N}$ fixed. By scaling invariance we may assume that the determinant of $H_{t}$ vanishes along the geodesic. Integrating the upper bound in (2-8) over $X$ gives

$$
J\left(H_{t}\right)=0+\left(I_{\mu_{0}} \circ \mathrm{FS}\right)\left(H_{t}\right) \leq C t+D .
$$


Now, let $f(t)=\mathscr{G}\left(H_{t}\right)$. Since by assumption $f$ is convex and 0 is a critical point, we have $d f / d t \geq 0$ for all $t$. Hence, if we fix some number $\epsilon>0$, then

$$
f(t) \geq f(0)+\int_{\epsilon}^{t}(d f / d s) d s .
$$

But by the assumption on strict convexity the latter integrand is bounded from below by some $\delta>0$. All in all this shows that

which finishes the proof.

$$
\varphi\left(H_{t}\right) \geq \delta t-A \geq \frac{\delta}{C} J\left(H_{t}\right)-A^{\prime},
$$

Large $\boldsymbol{k}$ asymptotics. Next, we will recall the following proposition, which is the link between the Bergman iteration and the Kähler-Ricci flow. It is essentially due to Bouche and Tian, apart from the uniformity with respect to $\phi$. In fact, a complete asymptotic expansion in powers of $k$ holds as was proved by Catlin and Zelditch and the uniformity can be obtained by tracing through the same arguments (as remarked in connection to Proposition 6 in [Donaldson 2001]). For references see the recent survey [Zelditch 2009].

Proposition 2.11. Assume that the volume form $\mu_{\phi}$ depends smoothly on $\phi$. Then the following uniform convergence for the corresponding Bergman function $\rho_{(k)}(\phi)$ holds: there is an integer $l$ such that

$$
\sup _{X}\left|\rho_{(k)}(\phi)-\frac{\left(d d^{c} \phi\right)^{n} / n !}{\mu_{\phi}}\right| \leq C / k
$$

for all weights $\phi$ such that $d d^{c} \phi$ is uniformly bounded from above in $\varphi^{l}$-norm with $d d^{c} \phi$ uniformly bounded from below by some fixed Kähler form.

\section{The Calabi-Yau setting}

First consider the absolute case where we assume given an ample line bundle $L \rightarrow X$. In this section we will the apply the general setting introduced in the previous section to the case when the measure $\mu$ is independent of $\phi$. We will assume that it is normalized, that is, a probability measure. We will mainly be interested in the case when $X$ is a Calabi-Yau manifold, which induces a canonical probability measure $\mu$ on $X$ defined by

$$
\mu=c_{n} \Omega \wedge \bar{\Omega}
$$

where $\Omega$ is any given holomorphic $n$-form trivializing the canonical line bundle $K_{X}$ and $c_{n}$ is a normalizing constant. In the relative Calabi-Yau setting, where each fiber is assumed to be a Calabi-Yau manifold, this hence yields a canonical smooth family of measures on the fibers.

For a fixed reference element $\phi_{0} \in \mathscr{H}_{L}$ we set

$$
I_{\mu}(\phi):=\int_{X}\left(\phi-\phi_{0}\right) \mu,
$$

which is equivariant under the usual actions of the additive group $\mathbb{R}: I_{\mu}(\phi+c)=I_{\mu}(\phi)+c$. Moreover, by definition the associated functional $-\mathscr{F}_{\mu}$ is coercive. 
3A. The relative Kähler-Ricci flow. The convergence on the level of Kähler forms in the following theorem is due to Cao (apart from the uniqueness, which was first shown by Calabi). We just observe that, since $\mu$ is normalized, the convergence of the flow also holds on the level of weights.

Theorem 3.1. The Kähler-Ricciflow on $\mathscr{H}_{L}$ with respect to $\mu$ exists for all times $t \in[0, \infty[$ and the solution $\phi_{t}$ is smooth on $X \times\left[0, \infty\left[\right.\right.$. Moreover, $\phi_{t} \rightarrow \phi_{\infty}$ uniformly in the $\mathscr{C}^{\infty}$-topology on $X$ when $t \rightarrow \infty$, where $\phi_{\infty}$ is the unique (modulo scaling) solution to the inhomogeneous Monge-Ampère equation (1-2). More precisely, all the analytical assumptions in Section 2 C are satisfied. In the Calabi-Yau case $\omega_{\infty}$ is Ricci flat.

Proof. As shown in [Cao 1985], $\omega_{t} \rightarrow \omega_{\infty}$ in the $\mathscr{C}^{\infty}$-topology. But then it follows from Proposition 2.4 that $\phi_{t} \rightarrow \phi_{\infty}$ uniformly in the $\mathscr{C}^{\infty}$-topology on $X$. The smoothness in the relative case was not stated explicitly in [Cao 1985] but follows from basic maximum principle arguments.

Preliminaries: Kodaira-Spencer classes and Weil-Petersson geometry. In this section we will assume that the base $S$ is one-dimensional and embedded as a domain in $\mathbb{C}$. Recall that the infinitesimal deformation of the complex structures on the smooth manifold $\mathscr{X}_{s}$ as $s$ varies is captured by the Kodaira-Spencer class $\rho\left(\frac{\partial}{\partial s}\right) \in H^{0,1}\left(T^{1,0} \mathscr{L}_{s}\right)$ [Voisin 2007]. When the fibers are Calabi-Yau manifolds the "size" of the deformation is measured by the (generalized) Weil-Petersson form $\omega_{\mathrm{WP}}$ (see [Fujiki and Schumacher 1990]) on the base $S$. It was extensively studied by Tian [1987] and Todorov [1989] when the base $S$ is a moduli space of Calabi-Yau manifolds and $\mathscr{X}$ is the corresponding Kuranishi family. The form $\omega_{\mathrm{WP}}$ is defined by

$$
\omega_{\mathrm{WP}}\left(\frac{\partial}{\partial s}, \frac{\partial}{\partial s}\right):=\left\|A_{C Y}\right\|_{\omega_{C Y}}^{2},
$$

where $A_{C Y}$ denotes the unique representative in the Kodaira-Spencer class $\rho(\partial / \partial s) \in H^{1,0}\left(T^{1,0} \mathscr{Q}_{s}\right)$ that is harmonic with respect to a given Ricci flat metric $\omega_{C Y}$ on $\mathscr{X}_{s}$ and the $L^{2}$-norm is computed with respect to this latter metric. Moreover, as shown in [Todorov 1989] the following formula holds:

$$
\left\|A_{C Y}\right\|_{\omega_{C Y}}^{2}=\frac{\partial^{2} \psi_{\Omega}}{\partial s \partial \bar{s}}, \quad \psi_{\Omega}(s):=\log i^{n^{2}} \int_{\mathscr{R}_{s}} \Omega_{s} \wedge \bar{\Omega}_{s},
$$

where $\Omega_{s}$ denotes a holomorphic family of nontrivial holomorphic $n$-forms on $\mathscr{X}_{s}$ for $s \in U$, a neighborhood of a fixed point $s$ in $S$. More generally, for an arbitrary smooth base $S$ the $(1,1)$-form $\omega_{\mathrm{WP}}$ on $S$ may be defined as the curvature of the holomorphic line bundle $\pi_{*}\left(K_{\chi / S}\right)$ on $S$. It is in the latter form that $\omega_{\mathrm{WP}}$ will appear in the proof of Theorem 3.3 below. In fact, the formula (3-1) may then be deduced from Theorem 3.3 (see Remark 3.7).

Next we will explain how, for a fixed base parameter $s$, a weight $\phi$ on the line bundle $\mathscr{L} \rightarrow \mathscr{X} \rightarrow S$ induces the following two objects:

- a $(0,1)$-form $A_{\phi}$ with values in $T^{1,0} \mathscr{X}_{s}$ representing the Kodaira-Spencer class $\rho\left(\frac{\partial}{\partial s}\right)$ in $H^{0,1}\left(T^{1,0} \mathscr{X}_{s}\right)$;

- a function $c(\phi)$ on $\mathscr{X}$ measuring the positivity (or lack of positivity) of $d d^{c} \phi$ on $\mathscr{X}$ in terms of the positivity of the restrictions of $d d^{c} \phi$ to the fibers $\mathscr{X}_{s}$. 
In fact $A_{\phi}$ will only depend on the family, parametrized by $s$, of two-forms $\omega_{s}$ obtained as the restrictions of the curvature form $\omega_{\phi}$ on $\mathscr{X}$ to all fibers $\mathscr{X}_{s}$, while $c(\phi)$ will depend on the whole form $\omega_{\phi}$.

Trivial fibrations. Assume that $\pi: \mathscr{X} \rightarrow S$ is a holomorphically trivial fibration, so that $\mathscr{X}$ is embedded in $\mathbb{C} \times X$ and that $\mathscr{L}=\pi^{*} L$ where $L \rightarrow X$ is an ample line bundle. Given a smooth family of weights $\phi(s, \cdot)$ on $L \rightarrow X$ with strictly positive curvature form $\omega_{\phi}^{X}:=d_{X} d_{X}^{c} \phi$ (for $s$ fixed) one obtains a smooth vector field $V_{\phi}$ of type $(1,0)$ as the "complex gradient" of $\partial_{s} \phi$ :

$$
\delta_{V_{\phi}} \omega_{\phi(s, \cdot)}^{X}=\bar{\partial}_{X}\left(\partial_{s} \phi\right)
$$

where $\delta_{V_{\phi}}$ denotes interior multiplication (i.e., contraction) with $V_{\phi}$. Now the $(0,1)$-form $A_{\phi}$ with values in $T^{1,0} X$ (for $s$ fixed) is simply defined by

$$
A_{\phi}:=-\bar{\partial}_{X} V_{\phi}
$$

Denote by $\omega_{t}^{X}$ the curvature forms on $X$ evolving with respect to the time parameter $t$ according to the Kähler-Ricci flow (for $s$ fixed). The Laplacian on $X$ with respect to $\omega_{t}^{X}$ will be denoted by $\Delta_{\omega_{t}^{X}}$. Given $\phi(s, \cdot)$ we define the following function on $\mathscr{X}$ :

$$
c(\phi):=\frac{1}{n}\left(d d^{c} \phi\right)^{n+1} /\left(d_{X} d_{X}^{c} \phi\right)^{n} \wedge i d s \wedge d \bar{s} .
$$

Note that, since $\omega_{\phi}^{X}>0$ on $X$, we have that $c(\phi)>0$ at $(s, x) \in \mathscr{X}$ if and only if $d d^{c} \phi>0$ at $(s, x)$.

General submersions. Next we turn to the case of a general holomorphic submersion $\pi: \mathscr{X} \rightarrow S$. Any given point in $\mathscr{X}$ has a neighborhood $\mathcal{U}$ such that the fibration $\pi: \mathscr{U} \rightarrow S$ is holomorphically trivial and the restriction $\mathscr{L}_{\mathscr{U}}$ is isomorphic to $\pi^{*} L$ over $U$. We introduce local holomorphic coordinates $(z, s)$ on $U$ such that $s$ defines a local holomorphic coordinate on $S$ and the projection $\pi: \cup \rightarrow S$ corresponds to $(z, s) \mapsto s$. Hence, the vector field $V_{\phi}$ defined above is locally defined, but in general not globally well-defined on $\mathscr{X}$. However, the expression (3-4) turns out to still be globally well-defined. For completeness we will give a proof of this well-known fact [Schumacher 2008; Fujiki and Schumacher 1990]:

Proposition 3.2. The $(0,1)$-form $A_{\phi}$ with values in $T^{0,1} \mathscr{X}_{s}$, locally defined by formula (3-4), is globally well-defined. It represents the Kodaira-Spencer class in $H^{0,1}\left(T^{1,0} \mathscr{X}_{s}\right)$.

Proof. Step 1. The locally defined expression

$$
W_{\phi}:=\frac{\partial}{\partial s}-V_{\phi}
$$

defines a global vector field on $\mathscr{X}$ of type $(1,0)$.

Indeed $W_{\phi}$ may be characterized as the horizontal lift of $\partial / \partial s$ with respect to the $(1,1)$-form $d d^{c} \phi$ on $\mathscr{X}$, which is nondegenerate along fibers. To see this first note that

$$
d \pi\left(W_{\phi}\right)=\frac{\partial}{\partial s} \quad \text { and } \quad d d^{c} \phi\left(W_{\phi}, \operatorname{ker} d \pi\right)=0 .
$$

The first point is trivial and the second one follows from a direct calculation: locally we may decompose

$$
d d^{c} \phi=d_{z} d_{z}^{c} \phi+\phi_{s \bar{s}} d s \wedge \bar{d} s+\left(\bar{\partial}_{z} \phi_{s}\right) \wedge d s+\left(\partial_{z} \phi_{s}\right) \wedge d \bar{s}
$$


Hence, for any fixed index $i$,

$$
d d^{c} \phi\left(W_{\phi}, \frac{\partial}{\partial \bar{z}_{i}}\right)=-d_{z} d_{z}^{c} \phi\left(V_{\phi}, \frac{\partial}{\partial \bar{z}_{i}}\right)+0+\left(\frac{\partial}{\partial \bar{z}_{i}} \phi_{s}\right)+0=0,
$$

using the definition (3-3) of $V_{\phi}$ in the last step. Finally, note that the properties (3-6) determine $W_{\phi}$ uniquely: if $W^{\prime}$ is another local vector field satisfying (3-6) then clearly $Z:=W_{\phi}-W^{\prime}$ satisfies

$$
d \pi(Z)=0 \quad \text { and } \quad d d^{c} \phi(Z, \operatorname{ker} d \pi)=0 .
$$

In particular, $Z$ is tangential to the fibers and $d d^{c} \phi(Z, \bar{Z})=0$. But since $d d^{c} \phi$ is assumed to be nondegenerate along the fibers it follows that $Z=0$.

Step 2. $A_{\phi}(s)=\left(\bar{\partial} W_{\phi}\right) \mathscr{x}_{s}$ and $A_{\phi}(s)$ represents the Kodaira-Spencer class in $H^{0,1}\left(T^{1,0} \mathscr{X}_{s}\right)$.

The first formula above follows immediately from a local computation and the second one then follows directly from the definition of the Kodaira-Spencer class (where $W_{\phi}$ may be taken as any smooth lift to $T^{1,0} \mathscr{L}$ of the vector field $\partial / \partial s$ [Voisin 2007]).

As for the function $c(\phi)$ defined by formula (3-5) it is still well-defined as we have fixed an embedding of $S$ in $\mathbb{C}$.

Conservation of positivity along the relative Kähler-Ricci flow. Next comes one of the main results of the present paper:

Theorem 3.3. Let $\pi: \mathscr{L} \rightarrow S$ be a proper holomorphic submersion with Calabi-Yau fibers and let $\mathscr{L}$ be a relatively ample line bundle over $\mathscr{X}$. Assume that the base $S$ is a domain in $\mathbb{C}$. The following equation holds along the corresponding relative Kähler-Ricci flow:

$$
\left(\frac{\partial}{\partial t}-\Delta_{\omega_{t}^{X}}\right) c(\phi)=\left|A_{\phi}\right|_{\omega_{t}^{X}}^{2}-\left\|A_{C Y}\right\|_{\omega_{C Y}^{X}}^{2} .
$$

Proof. Since it will be enough to prove the identity at a fixed point $x$ in $X$ in some local holomorphic coordinates and trivializations we may as well assume that $\omega_{\phi}$ is the Euclidean metric at the point $x$, that is, that the complex Hessian matrix $\left(\partial^{2} \phi / \partial z_{i} \bar{\partial} z_{j}\right)$ is the identity for $z=0$ (corresponding to the fixed point $x$ in $X$ ). Moreover, we may assume that locally the holomorphic $n$-form $\Omega$ may be expressed as $\Omega=d z_{1} \wedge \cdots \wedge d z_{n}$. Partial derivatives with respect to $s$ will be indicated by a subscript $s$ and partial derivatives with respect to $z_{i}$ and $\bar{z}_{j}$ by subscripts $i$ and $\bar{j}$ respectively. If $h=\left(h_{i j}\right)$ is a Hermitian matrix we will write $\left(h^{i j}\right)$ for the matrix $\bar{H}^{-1}$. The summation convention according to which repeated indices are to be summed over will be used. Next, we turn to the proof of the theorem which is based on a direct and completely elementary calculation.

Step 1. The following formula holds in the case of a holomorphically trivial fibration:

$$
\frac{\partial}{\partial t} c(\phi)=\phi_{i \bar{i} \bar{s} \bar{s}}+\phi_{s \bar{i}} \overline{\phi_{s \bar{j}}} \phi_{i \bar{j} k \bar{k}}-\phi_{i \bar{j} s} \overline{\phi_{i \bar{j} s}}-\phi_{s \bar{i}} \overline{\phi_{s \bar{j}}} \phi_{i k \bar{l}} \phi_{j \bar{k} l}-2 \Re\left(\phi_{k \bar{k} s \bar{i}} \overline{\phi_{s i}}\right)+2 \Re\left(\phi_{k \bar{l} s} \phi_{k \bar{l} \bar{i}} \overline{\phi_{s \bar{i}}}\right) \text {. }
$$

To see this first recall that

$$
c(\phi)=\phi_{s \bar{s}}-\Re\left(\phi_{s \bar{i}} \overline{\phi_{s \bar{j}}} \phi^{i \bar{j}}\right)
$$


and hence (using that $\phi_{i \bar{j}}=\delta_{i j}$ at $z=0$, so that $\partial \phi^{i \bar{j}} / \partial t=-\partial \phi_{\bar{j} i} / \partial t$ at $z=0$ )

$$
\frac{\partial}{\partial t} c(\phi)=\frac{\partial}{\partial t} \phi_{s \bar{s}}-2 \Re\left(\frac{\partial}{\partial t} \phi_{s \bar{i}}\right) \overline{\phi_{s \bar{i}}}+\left(\phi_{s \bar{i}} \overline{\phi_{s} \bar{j}}\right) \frac{\partial}{\partial t} \phi_{\bar{j} i}
$$

Using the definition of the relative Kähler-Ricci flow in the Calabi-Yau case and the simple fact that the linearization of $\psi \mapsto \log \operatorname{det}\left(\psi_{k \bar{l}}\right)$ at $\psi$ is given by $u \mapsto \Delta_{\omega_{\psi}} u$, where $\Delta_{\omega_{\psi}} u=\psi^{k \bar{l}} u_{k \bar{l}}$ is the Laplacian with respect to the Kähler metric $\omega_{\psi}$, hence gives

$$
\begin{aligned}
\frac{\partial}{\partial t} c(\phi) & =\left(\log \left(\operatorname{det} \phi_{i \bar{j}}\right)\right)_{s \bar{s}}-2 \Re\left(\left(\log \operatorname{det}\left(\phi_{k \bar{l}}\right)\right)_{s \bar{i}} \overline{\phi_{s i}}\right)+\left(\phi_{s i} \overline{\phi_{s \bar{j}}}\right)\left(\log \left(\operatorname{det} \phi_{k \bar{l}}\right)\right)_{i \bar{j}} \\
& =\left(\phi_{i \bar{j} s} \phi^{i \bar{j}}\right)_{\bar{s}}-2 \Re\left(\phi_{k \bar{l} s} \phi^{k \bar{l}}\right)_{\bar{i}} \overline{\phi_{s \bar{i}}}+\left(\phi_{s \bar{i}} \overline{\phi_{s} \bar{j}}\right)\left(\phi_{i k \bar{l}} \phi^{k \bar{l}}\right)_{\bar{j}} \\
& =\phi_{i \bar{i} s \bar{s}}-\phi_{i \bar{j} s} \phi_{j \bar{i} \bar{s}}-2 \Re\left(\phi_{k \bar{k} s \bar{i}} \overline{\phi_{s \bar{i}}}-\phi_{k \bar{l} s} \phi_{l \bar{k} \bar{i}} \overline{\phi_{s \bar{i}}}\right)+\left(\phi_{s \bar{i}} \overline{\phi_{s} \bar{j}}\right)\left(\phi_{i \bar{j} k \bar{k}}-\phi_{i k \bar{l}} \phi_{j \bar{k} l}\right)
\end{aligned}
$$

(again using $\phi_{i \bar{j}}=\delta_{i j}$ at $z=0$ ), finishing the proof of Step 1 .

Step 2. The following formula holds in the case of a trivial fibration:

$$
\begin{array}{r}
c(\phi)_{k \bar{k}}=\phi_{k \bar{k} s \bar{s}}+\left(\phi_{s \bar{i}} \overline{\phi_{s \bar{j}}}\right)\left(\phi_{k \bar{k} j \bar{i}}\right)-2\left(\phi_{s \bar{i}} \overline{\phi_{s \bar{j}}}\right) \phi_{\bar{k} \bar{i} m} \phi_{k \bar{m} j}-\phi_{k s \bar{i}} \overline{\phi_{k s \bar{i}}}-\overline{\phi_{\bar{k} s \bar{i}}} \phi_{\bar{k} s \bar{i}}+2 \Re\left(\phi_{k s \bar{i}} \overline{\phi_{s \bar{j}}} \phi_{\bar{k} j \bar{i}}\right) \\
+2 \Re\left(\overline{\phi_{\bar{k} s \bar{i}}} \phi_{s \bar{j}}\right) \phi_{\bar{k} j \bar{i}}-2 \Re \mathfrak{R} \phi_{\bar{k} k s \bar{i}} \overline{\phi_{s \bar{i}}} .
\end{array}
$$

To see this we first differentiate $c(\phi)$ with respect to $z_{k}$ to get

$$
c(\phi)_{k}=\phi_{k s \bar{s}}-\left[\left(\phi_{s \bar{i}} \overline{\phi_{s \bar{j}}}\right)_{k} \phi^{i \bar{j}}+\left(\phi_{s \bar{i}} \overline{\phi_{s} \bar{j}}\right)\left(\phi^{i \bar{j}}\right)_{k}\right]=\phi_{k s \bar{s}}-\left(\phi_{k s \bar{i}} \overline{\phi_{s} \bar{j}}+\overline{\phi_{\bar{k} s \bar{i}}} \phi_{s \bar{j}}\right) \phi^{i \bar{j}}-\left(\phi_{s \bar{i}} \overline{\phi_{s} \bar{j}}\right)\left(\phi^{i \bar{j}}\right)_{k} .
$$

Next, note that if $h$ is a function with values in the space of Hermitian matrices and $\partial$ a derivation satisfying the Leibniz rule, then

$$
\partial\left(h^{-1}\right)=-h^{-1}(\partial h) h^{-1} .
$$

In particular, if $h(0)=I$ then the following holds at 0 :

$$
\left(\bar{h}^{-1}\right)_{k \bar{k}}=-\bar{h}_{k \bar{k}}+\left(\bar{h}_{\bar{k}} \bar{h}_{k}+\bar{h}_{k} \bar{h}_{\bar{k}}\right) .
$$

Applying this to $h=\left(\phi_{i} \bar{j}\right)$ (when expanding the term $A$ below) gives

$$
\begin{aligned}
c(\phi)_{k \bar{k}} & \left.=\phi_{k \bar{k} s \bar{s}}-\left(\left[\left(\phi_{k s \bar{i}} \overline{\phi_{s \bar{i}}}\right)_{\bar{k}}+\overline{\left(\phi_{\bar{k} s \bar{i}}\right.} \phi_{s \bar{i}}\right)_{\bar{k}}\right]-\left(\phi_{k s \bar{i}} \overline{\phi_{s \bar{j}}}+\overline{\phi_{\bar{k} s \bar{i}}} \phi_{s j}\right) \phi_{\bar{k} i \bar{j}}\right)-A \\
& =\phi_{k \bar{k} s \bar{s}}-\left(\left[\phi_{\bar{k} k s \bar{i}} \overline{\phi_{s \bar{i}}}+\phi_{k s \bar{i}} \overline{\phi_{k s \bar{i}}}+\overline{\phi_{k \bar{k} s \bar{i}}} \phi_{s \bar{i}}+\overline{\phi_{\bar{k} s \bar{i}}} \phi_{\bar{k} s \bar{i}}\right]-\left(\phi_{k s \bar{i}} \overline{\phi_{s \bar{j}}}+\overline{\phi_{\bar{k} s \bar{i}}} \phi_{s \bar{j}}\right) \phi_{\bar{k} i \bar{j}}\right)-A,
\end{aligned}
$$

where

$$
\begin{aligned}
A & :=\left(\phi_{s \bar{i}} \overline{\phi_{s \bar{j}}}\right)_{\bar{k}}\left(\phi^{i \bar{j}}\right)_{k}+\left(\phi_{s \bar{i}} \overline{\phi_{s \bar{j}}}\right)\left(\phi^{i} \bar{j}\right)_{k \bar{k}}=-\left(\phi_{s \bar{i}} \overline{\phi_{s \bar{j}}}\right)_{\bar{k}} \phi_{k j \bar{i}}+\left(\phi_{s \bar{i}} \overline{\phi_{s \bar{j}}}\right)\left(-\phi_{k \bar{k} j \bar{i}}+2 \Re\left(\phi_{\bar{k} \bar{i} m} \phi_{k \bar{m} j}\right)\right) \\
& =-\left(\phi_{s \bar{i} \bar{k}} \overline{\phi_{s \bar{j}}}+\phi_{s \bar{i}} \overline{\phi_{s \bar{j} k}}\right) \phi_{k j \bar{i}}-\left(\phi_{s \bar{i}} \overline{\phi_{s \bar{j}}}\right)\left(\phi_{k \bar{k} j \bar{i}}+2 \Re\left(\phi_{\bar{k} \bar{i} m} \phi_{k \bar{m} j}\right)\right) .
\end{aligned}
$$

Hence,

$$
\begin{array}{r}
c(\phi)_{k \bar{k}}=\phi_{k \bar{k} s \bar{s}}-\left[\phi_{\bar{k} k s \bar{i}} \overline{\phi_{s \bar{i}}}+\phi_{k s \bar{i}} \overline{\phi_{k s \bar{i}}}+\overline{\phi_{k \bar{k} s \bar{i}}} \phi_{s \bar{i}}+\overline{\phi_{\bar{k} s \bar{i}}} \phi_{\bar{k} s \bar{i}}\right]+\left(\phi_{k s \bar{i}} \overline{\phi_{s \bar{j}}}+\overline{\phi_{\bar{k} s \bar{i}}} \phi_{s \bar{j}}\right) \phi_{\bar{k} j \bar{i}} \\
+\left(\phi_{s \bar{i} \bar{k}} \overline{\phi_{s \bar{i}}}+\phi_{s \bar{i}} \overline{\phi_{s \bar{i} k}}\right) \phi_{k j \bar{i}}+\left(\phi_{s \bar{i}} \overline{\phi_{s \bar{j}}}\right) \phi_{k \bar{k} j \bar{i}}-2 \Re\left(\phi_{s \bar{i}} \overline{\phi_{s \bar{j}}} \phi_{\bar{k} \bar{i} m} \phi_{k \bar{m} j}\right),
\end{array}
$$


which finishes the proof of Step 2.

Step 3: End of proof of the theorem for a trivial fibration. Subtracting the formulas from the previous steps gives, due to the cancellation of several terms,

$$
\frac{\partial}{\partial t} c(\phi)-c(\phi)_{k \bar{k}}=\phi_{s \bar{m} \bar{k}} \overline{\phi_{s \bar{m} \bar{k}}}+\left(\phi_{s \bar{i}} \overline{\phi_{s \bar{j}}}\right) \phi_{\bar{k} \bar{i} \bar{m}} \phi_{k \bar{m} j}-2 \Re\left(\overline{\phi_{s \bar{k} \bar{m}}} \phi_{s \bar{l}} \phi_{\bar{k} l \bar{m}}\right)=\sum_{m, k}\left|\phi_{s \bar{m} \bar{k}}-\sum_{l} \phi_{s \bar{l}} \phi_{\bar{k} \bar{m} l}\right|^{2} .
$$

Finally, note that

$$
\phi_{s \bar{m} \bar{k}}-\sum_{l} \phi_{s \bar{l}} \phi_{\bar{k} \bar{m} l}=\left(\phi_{s \bar{m}}\right)_{\bar{k}}-\left(\phi_{\bar{m} l}\right)_{\bar{k}} \sum_{l} \phi_{s \bar{l}}=\left(\phi_{s \bar{l}} \phi^{m \bar{l}}\right)_{\bar{k}}=\left(V_{m}\right)_{\bar{k}}
$$

(using $\phi_{i \bar{j}}=\delta_{i j}$ at $\left.z=0\right)$, where $V=\left(V_{1}, \ldots, V_{n}\right)$ is the $(0,1)$-vector field (3-3) expressed in local normal coordinates. This hence finishes the proof of the theorem in the case of a trivial fibration.

Step 4. We show that (3-7) holds for a general holomorphic submersion. As recalled above, any given point $P=\left(x, s_{0}\right)$ in $\mathscr{X}$ has a neighborhood $U$ such that the fibration $\pi: \mathscr{U} \rightarrow \pi(\mathcal{U})$ (where we after shrinking $S$ may assume that $\pi(U)=S$ ) is holomorphically trivial and the restriction $\mathscr{L}_{\mid \mho}$ is isomorphic to $\pi^{*} L$ over $\mathcal{U}$. We denote by $(z, s)$ a choice of holomorphic coordinates on $U$ trivializing the fibration. Moreover, when $\mathscr{X} \rightarrow S$ is a relative Calabi-Yau manifold we may furthermore choose $(z, s)$ with the property that there is a family $\Omega_{s}$ of nowhere vanishing holomorphic $n$-forms on the fibers $\mathscr{X}_{s}$ such that the restriction of $\Omega_{s}$ to $\mathcal{U}_{s}\left(:=\mathcal{U} \cap \mathscr{L}_{s}\right)$ coincides with the restriction of $d z:=d z_{1} \wedge \cdots \wedge d z_{n}$ to $u_{s}$. Indeed, first observe that we may choose $\Omega_{s}$ so that $\Omega_{s}=f_{s}(z) d z$ on $\mathcal{U}$, where $f(z, s):=f_{s}(z)$ is holomorphic in $(z, s)$ and invertible, with respect to any given holomorphic coordinates $(z, s)$ as above. This amounts to the well-known fact that the direct image sheaf $\pi_{*}\left(K_{\chi / S}\right)$ naturally defines a holomorphic line bundle on $S$ or equivalently that any $\Omega_{s_{0}}$ may be extended to $\Omega_{s}$ such that $\Omega_{s} \wedge d s$ is a holomorphic $(n+1)$-form on $\mathscr{X}$ (which for example follows from the Ohsawa-Takegoshi extension theorem; see [Berndtsson 2009a] for a more general setting). We may now (after perhaps shrinking $U$ again) write $f(z, s)=\partial g(z, s) / \partial z_{1}$ for some holomorphic functions $g$ on $U$ and define new holomorphic coordinates $(\zeta, s)$ on $U$ (after perhaps again shrinking $U$ ) by letting $\zeta_{i}:=g$ for $i=1$ and $\zeta_{i}:=z_{i}$ for $i>1$. By construction we then have $\Omega_{s \mid u_{s}}=d \zeta_{\mid u_{s}}$, as desired.

We can now repeat the previous local computation; the only new contribution comes from the derivatives on the local function $\psi_{\Omega}(s)$ defined by formula (3-2), which appear in the definition of the relative KählerRicci flow (1-3) in the Calabi-Yau case. Indeed, locally this latter flow may be written as

$$
\frac{\partial \phi}{\partial t}=\log \operatorname{det}\left(\phi_{k \bar{l}}\right)-\psi_{\Omega}(s)
$$

and the only new contribution to the previous calculations hence come from the term $-\left(\psi_{\Omega}(s)\right)_{s \bar{s}}$ which appears in the calculation of $(\partial \phi / \partial t)_{s \bar{s}}$. Combining formulae (3-1), (3-2) hence proves that (3-7) holds locally on $\mathscr{X}$. Since all objects appearing in the formula are globally well-defined, this finishes the proof of Step 4. 
Now the maximum principle for parabolic equations [Protter and Weinberger 1967] implies the following:

Corollary 3.4. Let $\mathscr{L} \rightarrow \mathscr{X} \rightarrow S$ be a line bundle over a fibration as in the previous theorem.

- If the fibration is holomorphically trivial, then the function $c(t):=\inf _{X} c(\phi)$ is, for a fixed value on $s$, increasing along the relative Kähler-Ricci flow and hence the flow preserves (semi)positivity of the curvature of $\phi$.

- For a holomorphically trivial fibration $\mathscr{X}=X \times S$, with $\mathscr{L}$ the pull-back of an ample line bundle $L \rightarrow X$, the flow improves the positivity of a generic initial weight in the following sense: if $\phi_{0}$ is a semipositively curved weight on $\mathscr{L}$ over $X \times S$ such that $\partial \phi / \partial s$ does not vanish identically on $X \times\{s\}$ for any $s$, then $\phi_{t}$ is strictly positively curved on $X \times S$ for $t>0$.

- In the general case the (semi)positivity of the curvature of the weight on $\phi-t \psi_{\Omega}$ on the $\mathbb{R}$-line bundle $\mathscr{L}-t K_{\mathscr{L} / S}$ is preserved under the flow; that is,

$$
d d^{c} \phi_{t} \geq-t \omega_{\mathrm{WP}}
$$

for all $t$ (and similarly in the strict case).

Proof. The first and third points follow from the maximum principle exactly as in the proof of Corollary 4.9 below. The second point is proved as follows: If strict positivity does not hold then one concludes (see the proof of Corollary 4.9 below) that $-A_{\phi_{0}}=\bar{\partial}_{X} V_{\phi_{0}}$ vanishes identically on $X$ for some $s_{0}$; that is, the corresponding vector field $V_{\phi_{0}}$ defined by (3-3) is holomorphic on $X$. But, it is a well-known fact that any such holomorphic vector field $V^{1,0}$ vanishes identically when $X$ is a Calabi-Yau manifold and hence $\partial \phi / \partial s$ vanishes identically on $X \times\left\{s_{0}\right\}$, giving a contradiction. The vanishing of $V^{1,0}$ may be proved as follows: by a Bochner-Weitzenbock formula $V^{1,0}$ is covariantly constant with respect to any Ricci flat metric on $X$. Moreover, the imaginary part $V_{I}$ satisfies $\omega_{\phi_{0}}\left(V_{I}, \cdot\right)=d f$ for some real smooth function $f$. But since $\omega_{\phi_{0}}^{X}>0$ on $X \times\left\{s_{0}\right\}$ the latter equation forces the vanishing of $V_{I}$ at any point where $f$ achieves it maximum and hence $V_{I} \equiv 0$ on $X$. Similarly, the real part $V_{R}$ of $V^{1,0}$ vanishes identically (by replacing $d f$ with $d^{c} f$ ).

Of course, in the case of an infinitesimally nontrivial fibration the inequality in the previous corollary is useless for the limit $\phi_{\infty}$, but its interest lies in the fact that it gives a lower bound on the (possible) loss of positivity along the relative Kähler-Ricci flow, which is independent of the initial data.

Remark 3.5. Throughout the paper we assume, for simplicity, that the initial weight $\phi_{0}$ has relatively positive curvature, when restricted to the fibers of the $\mathscr{X}$. But, as in the previous corollary, we do allow $\phi_{0}$ to have merely semipositive curvature over the total space $\mathscr{X}$. However, using recent developments for the Kähler-Ricci flow [Song and Tian 2009] the relative Kähler-Ricci flows are actually well-defined for any smooth weight $\phi_{0}$ which has merely relatively semipositive curvature and $\phi_{t}$ becomes relatively positively curved for any $t>0$. Using this result the previous corollary can be seen to be valid for a general semipositively curved initial weight $\phi_{0}$. Even more generally, as shown in [Song and Tian 2009], 
the flow is well-defined for any (possibly singular) $\phi_{0}$ with positive curvature current such that $\phi_{0}$ is locally bounded and the Monge-Ampère measure $\left(d d^{c} \phi_{0}\right)^{n}$ has local densities in $L^{p}$ for some $p>1$.

Evolution of the curvature of the top Deligne pairing. For a general smooth base $S$ (i.e., not necessarily embedded in $\mathbb{C}$ ) the weight $\phi$ on $L$ naturally induces a closed $(1,1)$-form $\Theta_{\phi}(s)$ on $S$ expressed as

$$
\Theta_{\phi}:=\pi_{*}\left(\left(d d^{c} \phi\right)^{n+1} /(n+1) !\right) .
$$

Equivalently, for any local holomorphic curve $C \subset S$ with tangent vector $\partial / \partial s \in T S$,

$$
\Theta_{\phi}\left(\frac{\partial}{\partial s}, \frac{\partial}{\partial \bar{s}}\right):=\int_{\chi_{s}} c(\phi) \omega_{\phi}^{n} / n !
$$

where $s \in C$ and $\pi$ is the induced map $\pi: \mathscr{X} \rightarrow C$. Geometrically, the form $\Theta_{\phi}$ on $S$ may be described as the curvature of the Hermitian holomorphic line bundle $(\mathscr{L}, \phi)^{n+1}$ over $S$ defined as the top Deligne pairing of the Hermitian holomorphic line bundle $(\mathscr{L}, \phi) \rightarrow \mathscr{X} \rightarrow S$ (see [Deligne 1987]; the relevance of Deligne pairings for Kähler geometry has been emphasized by Phong and Sturm [2004]). The form $\Theta_{\phi}$ also appears as a multiple of the curvature of the Quillen metric on the determinant of the direct image of a certain virtual vector bundle over $\mathscr{X}$ (see [Fujiki and Schumacher 1990] and references therein).

Similarly, one can define a $(1,1)$-form $\omega_{\mathrm{WP}_{\phi}}$ on $S$ depending on $\phi$ by letting

$$
\omega_{\mathrm{WP}_{\phi}}\left(\frac{\partial}{\partial s}, \frac{\partial}{\partial \bar{s}}\right):=\int_{\chi_{s}}\left|A_{\phi}(s)\right|^{2} \omega_{\phi}^{n} / n !
$$

It can be checked that this yields a well-defined $(1,1)$-form on $\mathscr{X}$. Anyhow this latter fact is also a consequence of the following corollary of the previous theorem.

Corollary 3.6. We make the same assumptions as in the previous theorem. Let $\Theta_{\phi_{t}}$ be the curvature form on $S$ of the top Deligne pairing of $(\mathscr{L}, \phi) \rightarrow \mathscr{X} \rightarrow S$, where $\phi_{t}$ evolves according to the relative Kähler-Ricci flow in the Calabi-Yau case. Then

$$
\frac{\partial}{\partial t} \Theta_{\phi}(s)=-\pi_{*}\left(R_{\omega_{\phi}^{X}}\left(d d^{c} \phi\right)^{n+1} /(n+1) !\right)+\omega_{\mathrm{WP}_{\phi_{t}}}-\omega_{\mathrm{WP}},
$$

where $R_{\omega_{\phi}^{X}}$ denotes the fiberwise scalar curvature of the metric $\omega_{\phi}$.

Proof. We may without loss of generality assume that $S$ is embedded in $\mathbb{C}_{S}$. Then

$$
\frac{\partial}{\partial t} \int_{\chi_{s}} c(\phi) \omega_{\phi}^{n} / n !=\int_{\chi_{s}} \frac{\partial}{\partial t} c(\phi) \omega_{\phi}^{n} / n !+\int c(\phi) \frac{\omega_{\phi}^{n-1}}{(n-1) !} \wedge d d^{c} \frac{\partial}{\partial t} \phi .
$$

Now, by the definition of the Kähler-Ricci flow in the Calabi-Yau case,

$$
\frac{\omega_{\phi}^{n-1}}{(n-1) !} \wedge d d^{c} \frac{\partial}{\partial t} \phi=\frac{\omega_{\phi}^{n-1}}{(n-1) !} \wedge\left(-\operatorname{Ric}\left(\omega_{\phi}^{X}\right)\right)=:-R_{\omega_{\phi}^{X}} \omega_{\phi}^{n} /(n+1) !
$$

where we have used the definition of the (normalized) scalar curvature $R_{\omega_{\phi}^{X}}$ of the Kähler metric $\omega_{\phi}^{X}$ in the last step. Finally, integrating the formula in the previous theorem finishes the proof of the corollary. 
Remark 3.7. If the initial weight $\phi$ for the Kähler-Ricci flow is taken so that $\omega_{\phi}$ restricts to a Ricci flat metric on all fibers of $\mathscr{X}$, then $\phi$ is stationary for the Kähler-Ricci flow and hence the previous corollary (and the proof of the previous theorem) shows that

$$
\int_{\chi_{s}}\left|A_{\phi}(s)\right|^{2} \omega_{\phi}^{n} / n=\frac{\partial^{2} \psi_{\Omega}}{\partial s \partial \bar{s}}
$$

that is, $\omega_{\mathrm{WP}}=d_{s} d_{s}^{c} i^{n^{2}} \log \int_{\mathscr{x}_{s}} \Omega \wedge \bar{\Omega}$. Since, by Proposition 4.5 below, $A_{\phi}(s)$ is harmonic on each fiber $\mathscr{X}_{s}$ with respect to the Ricci flat restriction $\omega_{\phi}$, this implies the equivalence between (3-1) and (3-2).

3B. Quantization: The Bergman iteration on $\mathscr{H}_{L}$. In this section we will specialize and develop the general results in Section 2D to the present setting where we have fixed a family of probability measures $\mu_{s}$ (independent of $\phi$ ) on the fibers $\chi_{s}$.

Convergence and positivity of the Bergman iteration at a fixed level $k$. The following monotonicity properties were shown by Donaldson [2009] in the present setting.

Lemma 3.8. The functionals $-I_{\mu}$ and $\mathscr{L}^{(k)}$ are both increasing along the Bergman iteration on $\mathscr{H}_{L}$ with respect to $\mu$. Moreover, they are strictly increasing at $\phi_{m}^{(k)}$ unless $\phi_{m}^{(k)}$ is stationary.

Proof. Since $I_{\mu}$ is affine and in particular concave on the affine space of all smooth weights the lemma follows immediately from Lemma 2.6.

We can now prove the convergence of the Bergman iteration at a fixed level $k$ in the present setting.

Theorem 3.9. Let $L \rightarrow X$ be an ample line bundle and $\mu$ a fixed volume form on $X$ giving unit volume to $X$. Assume a smooth initial weight $\phi_{0}$ is given. For any given positive integer $k$ the following holds: in the large time limit, that is, when $m \rightarrow \infty$, the weights $\phi_{m}^{(k)}$ converge to $\phi_{\infty}^{(k)}$ in the $\mathscr{C}^{\infty}$-topology on $X$. Moreover, in the relative setting the convergence is locally uniform with respect to the base parameter $s$.

Proof. By the previous lemma $-I_{\mu}$ is increasing and by definition $-\mathscr{F}_{\mu}^{(k)}$ is coercive. Moreover, as shown in [Berman et al. 2009] balanced weights are unique modulo scaling and hence all the convergence criteria in Proposition 2.9 are satisfied.

Conservation of positivity. Recall that, given a relatively ample line bundle $\mathscr{L}$ over a fibration $\pi: \mathscr{L} \rightarrow S$ as above, the corresponding direct image bundle $\pi_{*}\left(\mathscr{L}+K_{\mathscr{L} / S}\right) \rightarrow S$ is the vector bundle such that the fiber over $s$ is naturally identified with the space $H^{0}\left(X, L+K_{X}\right)$ of all holomorphic $n$-forms $f$ on $X:=\mathscr{L}_{s}$ with values in $L:=\mathscr{L}_{X}$ (as is well-known this is indeed a vector bundle, as shown using vanishing theorems). Moreover, any given weight $\phi$ on $\mathscr{L}$ induces a Hermitian metric on $\pi_{*}\left(k \mathscr{L}+K_{\mathscr{X} / S}\right)$ whose fiberwise restriction will be denoted by $\operatorname{Hilb}_{L+K_{X}}(\phi)$ :

$$
\operatorname{Hilb}_{L+K_{X}}(\phi)(f, f):=i^{n^{2}} \int_{X} f \wedge \bar{f} e^{-\phi} .
$$

The point is that there is no need to specify an integration measure $\mu$ thanks to the twist by the relative canonical line bundle $K_{\mathscr{X} / S}$. We will have great use for the following recent results of Berndtsson. 
Theorem 3.10. Let $\pi: \mathscr{L} \rightarrow S$ be a proper holomorphic submersion and let $\mathscr{L}$ be a relatively ample line bundle over $\mathscr{X}$ equipped with a smooth weight with semipositive curvature. Then:

- [Berndtsson 2009a] The curvature of the Hermitian vector bundle over $S$ defined as the direct image bundle $\pi_{*}\left(\mathscr{L}+K_{\mathscr{L} / S}\right)$ is semipositive in the sense of Nakano (and in particular in the sense of Griffiths).

- (See [Berndtsson 2011, Theorem 1.2 and subsequent discussion].) The vector bundle $\pi_{*}\left(\mathscr{L}+K_{\mathscr{L} / S}\right)$ has strictly positive curvature in the sense of Griffiths if either the curvature form of $\phi$ is strictly positive over all of $\mathscr{X}$ or strictly positive along the fibers of $\pi: \mathscr{L} \rightarrow S$ and the fibration is infinitesimally nontrivial (i.e., the Kodaira-Spencer classes are nontrivial for all $s \in S$ ).

We will only use the following simple consequence of Theorem 3.10 (compare [Berndtsson 2009a; Berndtsson and Păun 2008b]):

Corollary 3.11. Under the assumptions in the first point of the previous theorem we have

$$
d d^{c}\left(\mathrm{FS}^{(k)} \circ \operatorname{Hilb}_{k \mathscr{L}+K_{\mathscr{L} / S}}\right)(\phi) \geq 0
$$

and the inequality is strict under the assumptions in the second point of the theorem.

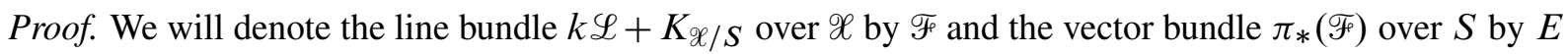
(and its dual by $E^{*}$ ). First note that the weight on $\mathscr{F}$ that we are interested in may be written as

$$
\left(\mathrm{FS} \circ \operatorname{Hilb}_{\mathscr{F}}\right)\left(s, x_{s}\right)=\log \sup _{f_{s} \in E_{s}} \frac{\left|f_{s}\left(x_{s}\right)\right|^{2}}{\left\|f\left(x_{s}\right)\right\|^{2}}=\log \left|\Lambda_{\left(s, x_{s}\right)}\right|^{2},
$$

where $\Lambda_{\left(s, x_{s}\right)}$ is the element in $E_{s}^{*} \otimes \mathscr{F}_{s}$ defined by

$$
\left(\Lambda_{\left(s, x_{s}\right)} f_{s}\right):=f_{s}\left(x_{s}\right) .
$$

Now let $t \mapsto\left(s_{t}, x_{s_{t}}\right)$ be a local holomorphic curve in $\mathscr{X}$ with $t \in \Delta$ (the unit-disc). Trivializing $\mathscr{F}$ in a neighborhood of the previous curve we may pull back $\Lambda_{\left(s, x_{s}\right)}$ to a holomorphic section $\Lambda_{t}$ of $E^{*}$ over the unit-disc and identify the weight defined by (3-10) with a function $\log \left|\Lambda_{t}\right|^{2}$ on $\Delta$. We have to prove that this latter function is (strictly) psh. But this follows from the following well-known fact: a vector bundle $E \rightarrow \Delta$ is (strictly) positive in the sense of Griffiths if and only if $\log \left(\left\|\Lambda_{t}\right\|^{2}\right)$ is (strictly) subharmonic on $\Delta$ where $\Lambda$ is any nontrivial holomorphic section of the dual vector bundle $E^{*}$. For example, to get the required (strict) subharmonicity one just notes that, after a standard computation,

$$
\left.\frac{\partial^{2} \log \left(\left\|\Lambda_{t}\right\|^{2}\right)}{\partial t \partial \bar{t}}\right|_{t=0} \geq-\frac{\Theta_{E^{*}}\left(\Lambda_{0}, \Lambda_{0}\right)}{\left\|\Lambda_{0}\right\|^{2}},
$$

where $\Theta_{E^{*}}$ at $t$ is the Hermitian endomorphism of $E_{t}^{*}$ representing the curvature of $E$. By the previous theorem $\Theta_{E}$ is (strictly) positive which is equivalent to $\Theta_{E *}$ being (strictly) negative and the corollary hence follows from the previous inequality.

We next obtain a "quantized" version of Corollary 3.4. 
Corollary 3.12. Let $\pi: \mathscr{L} \rightarrow S$ be a proper holomorphic submersion with Calabi-Yau fibers and let $\mathscr{L}$ be a relatively ample line bundle over $\mathscr{X}$.

- When $\pi$ is holomorphically trivial the relative Bergman iteration preserves semipositivity of the curvature of $\phi$.

- In the case of a general submersion with Calabi-Yau fibers,

$$
d d^{c} \phi_{(m)}^{(k)} \geq-\frac{m}{k} \omega_{\mathrm{WP}}
$$

for all $m$.

Proof. For simplicity first consider the case of a trivial fibration. Fix a holomorphic $n$-form $\Omega$ on $X:=\mathscr{X}_{0}$ trivializing $K_{X}$. Under the assumption that $\mathscr{L} \rightarrow S$ is holomorphically trivial $\Omega$ extends to a holomorphic $n$-form on all of $\mathscr{L}$ such that $\psi_{\Omega}:=\log \int_{\mathscr{C}_{s}} i^{n^{2}} \Omega \wedge \bar{\Omega}$ is independent of $s$. In this notation

$$
\operatorname{Hilb}^{(k)}(\phi(s, \cdot))(f, f):=\int_{\mathscr{C}_{s}}|f|^{2} e^{-\left(k \phi(s, \cdot)-\psi_{\Omega}(s)\right)} i^{n^{2}} \Omega \wedge \bar{\Omega} .
$$

Now consider the fiberwise isomorphism

$$
j: H^{0}(X, k L) \rightarrow H^{0}\left(X, k L+K_{X}\right), \quad j(f)=f \otimes \Omega,
$$

which clearly satisfies $\operatorname{Hilb}^{(k)}(\phi(s, \cdot))=e^{\psi_{\Omega}} j^{*} \operatorname{Hilb}_{L+K_{X}}(\phi(s, \cdot))$. This means that, up to a multiplicative constant independent of $s$, the map $j$ is an isometry when $H^{0}\left(X_{s}, k L+K_{X_{s}}\right)=H^{0}\left(\mathscr{L}, k \mathscr{L}+K_{\mathscr{L} / S}\right)_{\mathscr{X}_{s}}$ is equipped with its natural Hermitian product. In particular, by (3-9),

$$
d d^{c} \phi \geq 0 \Longrightarrow d d^{c}\left(\mathrm{FS}^{(k)} \circ \mathrm{Hilb}^{(k)}\right)(\phi) \geq 0 .
$$

Iterating hence proves the first point in the statement of the corollary. Finally, for a general submersion the same argument gives, but now taking into account the fact that $\psi_{\Omega}$ depends on $s$, that

$$
d d^{c} \phi \geq 0 \Longrightarrow d d^{c}\left(\mathrm{FS}^{(k)} \circ \operatorname{Hilb}^{(k)}\right)(\phi) \geq-d d^{c} \psi_{\Omega}(s) / k=-\omega_{\mathrm{WP}}(s) / k,
$$

using formula (3-2) in the last equality. Replacing $\phi$ with $\mathrm{FS}^{(k)} \circ \operatorname{Hilb}^{(k)}-\psi_{\Omega}(s)$ and iterating hence finishes the proof of the corollary.

Convergence towards the Kähler-Ricci flow. The following very simple proposition will turn out to be very useful:

Proposition 3.13. The following monotonicity holds for the Bergman iteration at level $k$ (with respect to $\mu$ ). Assume that $\phi_{m}^{(k)} \leq \psi_{m}^{(k)}$. Then $\phi_{m+1}^{(k)} \leq \psi_{m+1}^{(k)}$. In particular, the Bergman iteration decreases the distance in $\mathscr{H}_{L}$ defined with respect to the sup norm $d(\phi, \psi):=\sup _{X}|\phi-\psi|$.

Proof. By definition we have

$$
\phi_{m+1}^{(k)}=\phi_{m}^{(k)}+\frac{1}{k} \log \rho^{(k)}\left(k \phi_{m}^{(k)}\right)=\frac{1}{N_{k}} \sum_{i}\left|f_{i}\right|^{2} .
$$


By a well-known identity for Bergman kernels,

$$
\sum_{i=1}\left|f_{i}\right|^{2}(x)=\sup _{f \in H^{0}(X, k L)}\left(|f(x)|^{2} / \int_{X}|f|^{2} e^{-k \phi_{m}} d \mu\right) .
$$

But this latter expression is clearly monotone in $\phi_{m}$ proving the first statement of the proposition. As for the last statement just let $C:=\sup _{X}\left|\phi_{m}^{(k)}-\psi_{m}^{(k)}\right|$ so that

$$
\phi_{m}^{(k)} \leq \psi_{m}^{(k)}+C, \quad \psi_{m}^{(k)} \leq \phi_{m}^{(k)}+C .
$$

Applying the first statement of the proposition finishes the proof.

Remark 3.14. The previous proposition can be seen as a "quantum" analog of the corresponding result for the Kähler-Ricci flow (1-3), which follows directly from the maximum principle for the Monge-Ampère operator and its parabolic analogue.

Now we can prove the following theorem, which is one of the main results in this paper.

Theorem 3.15. Let $L \rightarrow X$ be an ample line bundle and $\mu$ a volume form on $X$ giving unit volume to $X$. Fix a smooth weight $\phi_{0}$ on $L$, whose curvature form is fiberwise strictly positive, and consider the corresponding Bergman iteration $\phi_{m}^{(k)}$ at level $k$ and discrete time $m$, as well as the Kähler Ricciflow $\phi_{t}-$ both defined with respect to $\mu$. Then there is a constant $C$ such that

$$
\sup _{X}\left|\phi_{m}^{(k)}-\phi_{m / k}\right| \leq C m / k^{2} .
$$

In particular, if $m_{k}$ is a sequence such that $m_{k} / k \rightarrow t$, then

$$
\phi_{m_{k}}^{(k)} \rightarrow \phi_{t}
$$

uniformly on $X$. Moreover, in the relative setting $C$ is locally bounded in the base parameter $s$ if $\mu$ depends smoothly on $s$.

Proof. Write $\psi_{k, m}=\phi_{m / k}$ and $F^{(k)}(\psi)=\frac{1}{k} \log \rho^{(k)}(\psi)$.

Step 1. We have $\psi_{k, m+1}-\psi_{k, m}=F^{(k)}\left(\psi_{k, m}\right)+O\left(1 / k^{2}\right)$ for all $(k, m)$, where the error term is uniform in $(k, m)$. (In the following we will take that as a definition of $O(1 / k)$, etc.)

To prove this we write the left-hand side as

$$
\frac{1}{k}\left(\frac{\phi_{m / k+1 / k}-\phi_{m / k}}{1 / k}\right)=\frac{1}{k}\left(\left.\frac{\partial \phi_{t}}{\partial t}\right|_{t=m / k}+O(1 / k)\right)
$$

using that $\left|\partial^{2} \phi_{t} / \partial^{2} t\right| \leq C$ on $X \times[0, T]$ by Theorem 3.1. More precisely, by the mean value theorem the error term $O(1 / k)$ may be written as

$$
\frac{1}{k} \frac{\partial^{2} \phi_{t}}{\partial^{2} t}(\zeta) / 2
$$

for some $\zeta \in[0,1 / k]$. 
Since $\phi_{t}$ evolves according to the Kähler-Ricci flow this means that

$$
\psi_{k, m+1}-\psi_{k, m}=\frac{1}{k} \log \left(\frac{\left(d d^{c} \phi_{m / k}\right)^{n} / n !}{\mu}\right)+O\left(1 / k^{2}\right) .
$$

But by Proposition 2.11 we have that

$$
F^{(k)}\left(\phi_{m / k}\right)=\frac{1}{k} \log \left(\frac{\left(d d^{c} \phi_{m / k}\right)^{n} / n !}{\mu}\right)+O\left(1 / k^{2}\right),
$$

where the error term is uniformly bounded in $(m, k)$ for $m / k \leq T$ by Theorem 3.1. In fact, as is well-known the uniform estimates (2-4) on the "space-derivatives" of $\phi_{t}$ in Theorem 3.1 also hold for all time-derivatives $d^{r} \phi_{t} / d^{r} t$ (and in particular for $r=1$ and $r=2$ used above). This is well-known and shown by differentiating the flow equation with respect to time and applying the maximum principle repeatedly. Hence, $T$ may be taken to be equal to infinity, which finishes the proof of Step 1.

Step 2. Given Step 1 and the fact that the Bergman iteration decreases the sup norm, we have

$$
\sup _{X}\left|\phi_{m}^{(k)}-\psi_{k, m}\right| \leq C m / k^{2} .
$$

We will prove this by induction over $m$ (for $k$ fixed), the statement being trivially true for $m=0$. By Step 1 there is a uniform constant $C$ such that

$$
\sup _{X}\left|\psi_{k, m+1}-\left(\psi_{k, m}+F^{(k)}\left(\psi_{k, m}\right)\right)\right| \leq C\left(1 / k^{2}\right)
$$

for all $(m, k)$. Now we fix the integer $k$ and assume as an induction hypothesis that (3-11) holds for $m$ with $C$ the constant in the previous inequality. By Proposition 3.13,

$$
\sup _{X}\left|\left(\psi_{k, m}+F^{(k)}\left(\psi_{k, m}\right)\right)-\left(\phi_{m}^{(k)}+F^{(k)}\left(\phi_{m}^{(k)}\right)\right)\right| \leq \sup _{X}\left|\psi_{k, m}-\phi_{m}^{(k)}\right| \leq C m / k^{2}
$$

with the same constant $C$ as above, using the induction hypothesis in the last step. Combining this estimate with the previous inequality gives

$$
\sup _{X}\left|\psi_{k, m+1}-\phi_{m+1}^{(k)}\right| \leq C m / k^{2}+C / k^{2},
$$

proving the induction step and hence Step 2.

Of course, it seems natural to expect that $\mathscr{C}^{\infty}$-convergence holds but we leave this problem for the future.

Combining the previous corollary with Theorem 3.15 and the variational principle in [Berman et al. 2009] (the $\mathscr{C}^{\infty}$-convergence rather uses [Keller 2009; Wang 2005]) now gives the following:

Corollary 3.16. The conservation of semipositivity of the curvature of $\phi_{t}$ in Corollary 3.4 holds. For a fixed initial data $\phi_{0}=\phi_{0}^{(k)} \in \mathscr{H}_{L}$ the following convergence results hold for the Bergman iteration $\phi_{m}^{(k)}$ :

- For any sequence $m_{k}$ such that $m_{k} / k \rightarrow \infty$ the convergence $\phi_{m_{k}}^{(k)} \rightarrow \phi_{\infty}$ holds in the $L^{1}$-topology on $X$. Moreover, if it is also assumed that $m_{k} / k^{2} \rightarrow 0$ then the convergence holds in the $C^{0}$-topology. 
- The balanced weights $\phi_{\infty}^{(k)}:=\lim _{m \rightarrow \infty} \phi_{m}^{(k)}$ at level $k$ converge, when $k \rightarrow \infty$, in the $\mathscr{C}^{\infty}$-topology, to the weight $\phi_{\infty}$ which is the large time limit of the corresponding Kähler-Ricci flow (and in particular a solution to the corresponding inhomogeneous Monge-Ampère equation).

In the relative case the convergence holds fiberwise locally uniformly with respect to the base parameter $s$. Proof. The first statement follows immediately by combining Theorem 3.15 and the previous corollary, since semipositivity is preserved under uniform limits of weights. Hence, we turn to the proof of the first point. It is based on the following inequalities:

$$
\limsup _{k \rightarrow \infty} I_{\mu}\left(\phi_{m_{k}}^{(k)}\right) \leq I_{\mu}\left(\phi_{\infty}\right), \quad \liminf _{k \rightarrow \infty} \mathscr{E}\left(\phi_{m_{k}}^{(k)}\right) \geq \mathscr{E}\left(\phi_{\infty}\right) .
$$

To prove these inequalities take a sequence $m_{k}^{\prime}$ such that $m_{k}^{\prime} / k \rightarrow t$ and $m_{k}^{\prime} \leq m_{k}$. By monotonicity (Lemma 3.8),

$$
I_{\mu}\left(\phi_{m_{k}}^{(k)}\right) \leq I_{\mu}\left(\phi_{m_{k}^{\prime}}^{(k)}\right) .
$$

Hence, letting $k \rightarrow \infty$ and using that $\phi_{m^{\prime} k}^{(k)} \rightarrow \phi_{t}$ uniformly (by Theorem 3.15) gives

$$
\limsup _{k \rightarrow \infty} I_{\mu}\left(\phi_{m_{k}}^{(k)}\right) \leq I_{\mu}\left(\phi_{t}\right) .
$$

Finally, letting $t \rightarrow \infty$ and using Theorem 3.1 proves the first inequality in (3-12). As for the second inequality in (3-12), it is similarly proved by noting that, by monotonicity,

$$
\mathscr{L}^{(k)}\left(\phi_{m_{k}^{\prime}}^{(k)}\right) \leq \mathscr{L}^{(k)}\left(\phi_{m_{k}}^{(k)}\right) .
$$

To proceed we will use that $\psi_{k} \rightarrow \psi$ uniformly in $\mathscr{H}_{L}$ implies that

$$
\mathscr{L}^{(k)}\left(\psi_{k}\right) \rightarrow \mathscr{E}(\psi) .
$$

To see this recall that this is well-known when $\psi_{k}=\psi$ for all $k$ (as follows for example from Proposition 2.11, saying that the convergence holds for the differentials $d \mathscr{L}^{(k)}$ and $d \mathscr{E}$; for more general convergence results see [Berman and Boucksom 2010]). But then the general case follows easily from the fact that $\mathscr{L}^{(k)}$ is monotone in the argument $\psi$ and scaling equivariant. Hence, letting $k \rightarrow \infty$ gives, since $\psi_{k}:=\phi_{m_{k}{ }_{k}}^{(k)} \rightarrow \phi_{t}$ uniformly, that

$$
\mathscr{E}\left(\phi_{t}\right) \leq \liminf _{k \rightarrow \infty} \mathscr{L}^{(k)}\left(\phi_{m_{k}}^{(k)}\right) .
$$

The proof of the second inequality in (3-12) is finished by using that (as shown in [Berman et al. 2009]), for any sequence $\left(\psi_{k}\right)$ in $\mathscr{H}_{L}$,

$$
\limsup _{k \rightarrow \infty} \mathscr{L}^{(k)}\left(\psi_{k}\right) \leq \liminf _{k \rightarrow \infty} \mathscr{E}\left(\psi_{k}\right) .
$$

Now, adding up the two inequalities in (3-12) gives

$$
\liminf _{k \rightarrow \infty} \mathscr{F}\left(\phi_{m_{k}}^{(k)}\right) \geq \mathscr{F}\left(\phi_{\infty}\right) .
$$


But then it follows from the variational results in [Berman et al. 2009] that

$$
\lim _{k \rightarrow \infty} \mathscr{F}\left(\phi_{m_{k}}^{(k)}\right)=\mathscr{F}\left(\phi_{\infty}\right)
$$

and

$$
d d^{c} \phi_{m_{k}}^{(k)} \rightarrow d d^{c} \phi_{\infty}
$$

in the weak topology of currents. Next, note that by the inequalities (3-12) the sequence $\phi_{m_{k}}^{(k)}$ is contained in a compact subset of $\mathscr{H}_{L}$ equipped with the $L^{1}$-topology (compare the proof of Lemma 2.3) and hence we may assume (perhaps after passing to a subsequence) that $\phi_{m_{k}}^{(k)} \rightarrow \psi$ in the $L^{1}$-topology. But then the convergence in (3-14) forces $\psi=\phi_{\infty}+C$ for some constant $C$. Hence, it will be enough to prove that $C=0$. To this end, note that combining (3-13) and the inequalities (3-12) shows that the latter inequalities are in fact equalities. In particular,

$$
\lim _{k \rightarrow \infty} \mathscr{E}\left(\phi_{m_{k}}^{(k)}\right)=\mathscr{E}\left(\phi_{\infty}\right)
$$

By the scaling equivariance of $\mathscr{E}$ it hence follows that $C=0$, which finishes the proof of the first point. If one assumes that $m_{k} / k^{2} \rightarrow 0$ then it follows immediately from combining Theorem 3.1 and Theorem 3.15 that the convergence holds uniformly on $X$, that is, in the $C^{0}$-topology.

To prove the second point in the statement of the corollary note that replacing $\phi_{m_{k}}^{(k)}$ by $\phi_{\infty}^{(k)}$ in the previous argument gives, just as before, that $\phi_{\infty}^{(k)} \rightarrow \phi_{\infty}$ in the $L^{1}$-topology. Moreover, since it was shown in [Keller 2009; Wang 2005] that the convergence of the corresponding curvature forms holds in the $\mathscr{C}^{\infty}$-topology this proves the second point.

\section{The (anti)canonical setting}

In this section we will consider another particular case of the general setting in Section 2 arising when the line bundle $L:=r K_{X}$ is ample, where $r=1$ or $r=-1$ (for any fiber $X$ of the fibration). Hence, $X$ is necessarily of general type in the former "positive" case and a Fano manifold in the latter "negative" setting. We will also refer to these two different settings as the $\pm K_{X}$-settings.

By the very definition of the canonical line bundle any weight $\phi$ on $\pm K_{X}$ determines a canonical scale-invariant probability measure $\mu_{ \pm}(\phi)$ on $X$, where

$$
\mu_{ \pm}(\phi):=e^{ \pm \phi} / \int_{X} e^{ \pm \phi}
$$

(with a slight abuse of notation), so that $\mu_{ \pm}(\phi+c)=\mu_{ \pm}(\phi)$. Equivalently, $\mu_{ \pm}(\phi)$ may be identified with the one-form on $\mathscr{H}_{ \pm K_{X}}$ obtained as the differential of the following functional $I_{ \pm}(\phi)$ on $\mathscr{H}_{ \pm K_{X}}$ :

$$
I_{ \pm}(\phi):= \pm \log \int_{X} e^{ \pm \phi}, \quad \mu_{ \pm}(\phi)=d I_{ \pm} .
$$

A characteristic feature of the $\pm K_{X}$-setting is that the antiderivative $I_{ \pm}$is canonically defined (i.e., not only up to scaling). As a consequence there is a canonical normalization condition for weights that will occasionally be used below, namely the condition that $I_{ \pm}(\phi)=0$. 
We will also have use, as before, for the equivariant functional

$$
\mathscr{F}_{ \pm}:=\mathscr{E}-I_{ \pm}
$$

where $\mathscr{E}$ is the functional defined in Section $2 \mathrm{~B}$ (with respect to a fixed reference weight in $\pm K_{X}$ ). ${ }^{1}$ Note that the critical points of $\mathscr{F}_{ \pm}$on $\mathscr{H}_{ \pm K_{X}}$ are the Kähler-Einstein weights $\phi$, that is, the weights such that $\omega_{\phi}$ is a Kähler-Einstein metric on $X$ (compare Theorem 4.1 below).

It will also be important to consider a nonnormalized variant of $\mu_{ \pm}(\phi)$ defined by

$$
\mu_{ \pm}^{\prime}(\phi):=e^{ \pm \phi}
$$

(which is the differential of the nonequivariant functional $\phi \mapsto \int e^{ \pm \phi}$ ). In the sequel we will refer to the two different settings defined by $\mu_{ \pm}(\phi)$ and $\mu_{ \pm}^{\prime}(\phi)$ as the normalized $\pm K_{X}$-setting and the nonnormalized $\pm K_{X}$-setting, respectively. It should be pointed out that it is the latter one which usually appears in the literature on the Kähler-Ricci flow (see for example [Cao 1985; Tian and Zhu 2007; Phong et al. 2007]).

4A. The relative Kähler-Ricci flow. According to the general construction in Section 2 each particular setting introduced above comes with an associated relative Kähler-Ricci flow. For future reference we will write out the fiberwise flow in the nonnormalized $\pm K_{X}$-setting in local holomorphic coordinates:

$$
\frac{\partial \phi}{\partial t}=\log \operatorname{det}\left(\frac{1}{\pi} \frac{\partial^{2} \phi}{\partial z_{i} \partial \overline{z_{j}}}\right) / n !-( \pm \phi) .
$$

The normalized and nonnormalized settings induce the same evolution of the fiberwise curvature forms $\omega_{t}$ :

$$
\frac{\partial \omega_{t}}{\partial t}=-\operatorname{Ric} \omega_{t}- \pm \omega_{t}
$$

in $c_{1}\left( \pm K_{X}\right) .^{2}$

In particular, if $\omega_{t}$ converges to $\omega_{\infty}$ in the large time limit, then $\omega_{\infty}$ is necessarily a Kähler-Einstein metric, which is of negative scalar curvature in the $K_{X}$-setting and positive scalar curvature in the $-K_{X}$-setting.

The main virtue of the Kähler-Ricci flow in the normalized setting as compared with the nonnormalized one is that the first one is convergent precisely when the flow of curvature forms $\omega_{t}$ is. On the other hand, as will be seen later the flow in the nonnormalized setting (and its quantized version) has better monotonicity and positivity properties.

Theorem 4.1. The Kähler-Ricci flow in the $\pm K_{X}$-settings always exists and is smooth on $X \times[0, \infty[$. More precisely, all the analytical assumptions in Section $2 C$ are satisfied. In the normalized $K_{X}$-setting it converges to a Kähler-Einstein metric of negative scalar curvature. In the $-K_{X}$-setting the flow converges to a Kähler-Einstein metric of positive scalar curvature under the assumptions that $H^{0}(T X)=0$ and $X$

\footnotetext{
${ }^{1}$ Note that $\mathscr{F}_{ \pm}$is minus the functional introduced in [Tian 2000].

${ }^{2}$ In the literature this latter flow of Kähler forms is sometimes referred to as the normalized Kähler-Ricci flow, as opposed to Hamilton's original flow, but our use of the term "normalized" is different and only applies on the level of weights on $L$.
} 
a priori admits a Kähler-Einstein metric. Furthermore, in the relative case the convergence is locally uniform with respect to the base parameter $s$.

Apart from the uniqueness statement, the first part of the previous theorem is due to Cao [1985]. The convergence on the level of Kähler metrics in the Fano case, that is, when $-K_{X}$ is ample, was proved by Perelman (unpublished) and Tian and Zhu [2007]. The convergence on the level of weights then follows directly from Proposition 2.4 and the known coercivity of the functionals $-\mathscr{F}_{ \pm}$; the coercivity of $-\mathscr{F}_{+}$ follows immediately from Jensen's inequality, while the coercivity of - $\mathscr{F}$ - was shown in [Phong et al. 2008], confirming a conjecture of Tian. The uniqueness in the difficult case of $-K_{X}$ is due to Bando and Mabuchi (for a comparatively simple proof see [Berman et al. 2009]).

Remark 4.2. The first key analytical ingredient in the proof of the convergence of the flow of Kähler metric $\omega_{t}$ in the Fano case (i.e., the $-K_{X}$-setting) is an estimate of Perelman saying that the Ricci potential $h_{t}$ of $\omega_{t}$, when suitably normalized, is always bounded along the Kähler-Ricci flow for $\omega_{t}$ (see [Tian and Zhu 2007; Phong et al. 2007]). In fact, in the present notation $h_{t}$ coincides (modulo signs) with the time derivative of $\phi_{t}$ evolving according to the normalized Kähler-Ricci flow in the $-K_{X}$-setting. The second key ingredient is the fact that the existence of a Kähler-Einstein metric implies that $-\mathscr{F}_{+}$is proper (and conversely [Tian 2000; Phong et al. 2008]). As is well-known there are, in general, obstructions to existence of Kähler-Einstein metrics in the $-K_{X}$-setting. According to a conjecture of Yau the existence of a Kähler-Einstein metric should be equivalent to a suitable notion of algebraic stability (in the sense of geometric invariant theory). From this point of view the properness (or coercivity) assumption on the functional $-\mathscr{F}_{+}$can be considered as an analytic stability [Tian 2000].

Definition. A weight $\phi_{\mathrm{KE}}$ on $\pm K_{X}$ will be called a normalized Kähler-Einstein weight if $I_{ \pm}\left(\phi_{\mathrm{KE}}\right)=0$, or equivalently if $e^{ \pm \phi_{\mathrm{KE}}}=\omega_{\mathrm{KE}}^{n} / n !$.

Hence, there is precisely one normalized Kähler-Einstein weight on $+K_{X}$ when it is ample. The following simple corollary of Theorem 4.1 and Remark 4.2 illustrates the difference between the normalized and nonnormalized settings.

Corollary 4.3. In the $+K_{X}$-setting the nonnormalized flow (4-1) always converges to the normalized Kähler-Einstein weight.

Proof. Write $\phi_{t}^{\prime}$ for the evolution under the Kähler-Ricci flow in the nonnormalized $K_{X}$-setting so that

$$
\phi_{t}^{\prime}=\phi_{t}+C_{t},
$$

where $C_{t}$ is a constant for each $t$. Since $\phi \mapsto\left(d d^{c} \phi\right)^{n}$ is invariant under scalings, comparing the two flow equations gives

$$
\frac{\partial C_{t}}{\partial t}=-C_{t}-I_{+}\left(\phi_{t}\right)
$$

Let $D_{t}:=C_{t}-I\left(\phi_{t}\right)$. Then we get

$$
\frac{\partial D_{t}}{\partial t}=-D_{t}+\epsilon_{t}, \quad \text { where } \epsilon_{t}:=\frac{\partial I_{+}\left(\phi_{t}\right)}{\partial t} .
$$


In the $+K_{X}$-setting Theorem 4.1 implies that $\epsilon_{t} \rightarrow 0$; it follows for elementary reasons that $D_{t} \rightarrow 0$. Indeed, assume for a contradiction that $D_{t}$ does not converge to 0 . Then $\partial \log \left|D_{t}\right| / \partial t \rightarrow-1$; that is, $\left|D_{t}\right| \leq C_{\delta} e^{-t(1-\delta)} \rightarrow 0$ for $0<\delta \ll 1$, giving a contradiction. Finally, in the nonnormalized $-K_{X^{-}}$ setting it was shown in [Phong et al. 2007] (building on [Chen and Tian 2002]) that there is a constant $c_{0}$ such that $\phi_{t}^{\prime}$ converges. But then it follows immediately from combining the scaling invariance of $\phi \mapsto\left(d d^{c} \phi\right)^{n}$ and the scaling equivariance of $\mu_{-}^{\prime}$ that the flow diverges exponentially for any other choice of constant $c_{0}$.

Remark 4.4. In the nonnormalized $-K_{X}$-setting (under the assumptions in the previous theorem) it was shown in [Phong et al. 2007] (building on [Chen and Tian 2002]) that the flow converges when the initial weight $\phi_{0}$ is replaced by $\phi_{0}+c_{0}$ for a unique constant $c_{0}$. The argument in the proof of the previous corollary then gives that for a generic initial weight the flow is divergent.

4B. Weil-Petersson geometry. As before we may in the following assume that the base $S$ is embedded in $\mathbb{C}$. In the relative $\pm K_{X}$-setting the (generalized) Weil-Petersson form $\omega_{\mathrm{WP}}$ on $S$ was introduced in [Koiso 1983] (see also [Fujiki and Schumacher 1990] for generalizations):

$$
\omega_{\mathrm{WP}}\left(\frac{\partial}{\partial s}, \frac{\partial}{\partial \bar{s}}\right):=\left\|A_{\mathrm{KE}}\right\|_{\omega_{\mathrm{KE}}}^{2},
$$

where $A_{\mathrm{KE}}$ denotes the unique representative in the Kodaira-Spencer class $\rho\left(\frac{\partial}{\partial s}\right) \in H^{0,1}\left(T^{1,0} \mathscr{X}_{s}\right)$ which is harmonic with respect to the Kähler-Einstein metric on $\mathscr{X}_{s}$ and the $L^{2}$-norm is computed with respect to this latter metric. In fact, as shown in [Fujiki and Schumacher 1990, Proposition 4.12], $A_{\mathrm{KE}}=-\bar{\partial} V_{\omega_{s}}$, where $V_{\omega_{s}}$ is the local vector field defined by formula (3-3). This is a consequence of the following proposition proved in [Fujiki and Schumacher 1990].

Proposition 4.5. Let $\pi: \mathscr{L} \rightarrow S$ be a proper holomorphic submersion and $\omega_{s}$ a smooth family of 2-forms on the fibers $\mathscr{D}_{s}$ such that $\omega_{s}$ is Kähler-Einstein on $\mathscr{X}_{s}$. Then $A_{\omega_{s}}$ is the unique element in $H^{0,1}\left(T \mathscr{X}_{s}\right)$ which is harmonic with respect to $\omega_{s}$.

Note that "harmonic" lifts of vector fields were previously used by Siu [1986] in the context of Weil-Petersson geometry.

Remark 4.6. When the relative dimension is one the space $H^{0,1}\left(T \mathscr{Q}_{s}\right)$ is isomorphic to $H^{1,0}\left(\left(T \mathscr{X}_{s}\right)^{*}\right)=$ $H^{0}\left(2 K_{\chi_{s}}\right)$ under Serre duality. Hence, the Weil-Petersson form as defined in terms of harmonic representatives then coincides with the metric on $\mathscr{X}$ introduced by Weil in the case when $\mathscr{X}$ is the universal family over Teichmüller space. As conjectured by Weil and subsequently proved by Ahlfors this latter $(1,1)$-form is closed and hence Kähler. In the higher-dimensional case, it was observed in [Fujiki and Schumacher 1990] that the Kähler property of $\omega_{\mathrm{WP}}$ as defined by (4-4) follows immediately from Corollary 4.10 below.

By an application of the implicit function theorem (in appropriate Banach spaces) the smoothness of the family $\omega_{S}$ (and of the associated normalized weight) in the previous proposition is automatic in the $+K_{X}$-case case, as well as in the $-K_{X}$ case if there are no nontrivial holomorphic vector fields tangential to the fibers of the fibration (see Theorem 6.3 in [Fujiki and Schumacher 1990]).

Now we can prove the following variant of Theorem 3.3. 
Theorem 4.7. Let $\pi: \mathscr{X} \rightarrow S$ be a proper holomorphic submersion. Assume that $\pm K_{\mathscr{X} / S}$ is relatively ample and that $\phi_{t}$ evolves according to the Kähler-Ricci flow in the nonnormalized setting. Then

$$
\frac{\partial c\left(\phi_{t}\right)}{\partial t}=\Delta_{\omega_{t}^{X}} c\left(\phi_{t}\right)- \pm c\left(\phi_{t}\right)+\left|A_{\phi_{t}}\right|_{\omega_{t}^{X}}^{2} .
$$

In particular, if $\phi_{\mathrm{KE}}$ is a fiberwise normalized Kähler-Einstein weight, then

$$
\Delta_{\omega_{\mathrm{KE}}} c\left(\phi_{\mathrm{KE}}\right)- \pm c\left(\phi_{\mathrm{KE}}\right)+\left|A_{\omega_{\mathrm{KE}}}\right|_{\omega_{\mathrm{KE}}^{X}}^{2}=0 .
$$

Proof. To simplify the notation we will only consider the $+K_{X}$-setting, but the proof in the $-K_{X}$ setting is essentially the same. We will just indicate the simple modifications of the proof of Theorem 3.3 which arise in the present setting.

Let us first consider the modifications to the calculation of the $t$-derivative of $c(\phi)$ that arise from the additional term $-\phi$ appearing in the calculation of the time derivative $\phi_{t}$, since now

$$
\frac{\partial}{\partial t} \phi_{t}=\log \operatorname{det}\left(\phi_{k \bar{l}}\right)-\phi
$$

in local coordinates. To this end we assume to simplify the notation that $X$ is one-dimensional (but the general argument is essentially the same). First recall that, according to formula (3-8),

$$
\frac{\partial}{\partial t} c(\phi)=\frac{\partial}{\partial t} \phi_{s \bar{s}}-\left[\left(\phi_{s \bar{z}} \overline{\phi_{s \bar{z}}}\right)_{t} \phi_{z \bar{z}}^{-1}-\left(\phi_{s \bar{z}} \overline{\phi_{s} \bar{z}}\right) \phi_{z \bar{z}}^{-2} \frac{\partial}{\partial t} \phi_{z \bar{z}}\right] .
$$

Hence, the additional contribution referred to above is of the form

$$
B:=(-\phi)_{s \bar{s}}-2 \Re\left(-\phi_{s \bar{z}} \overline{\phi_{s \bar{z}}}\right) \phi_{z \bar{z}}^{-1}+\phi_{s \bar{z}} \overline{\phi_{s \bar{z}}} \phi_{z \bar{z}}^{-2}\left(-\phi_{z \bar{z}}\right)=\left(-\phi_{s \bar{s}}\right)+2\left|\phi_{s \bar{z}}\right|^{2} \phi_{z \bar{z}}^{-1}-\phi_{s \bar{z}} \overline{\phi_{s \bar{z}}} \phi_{z \bar{z}}^{-1}=-c(\phi) \text {. }
$$

Hence, the local calculations in the Calabi-Yau case give that

$$
\frac{\partial}{\partial t} c(\phi)=\Delta_{\omega_{t}} c(\phi)-c(\phi)+\left|A_{\phi}\right|_{\omega_{t}^{X}}^{2} .
$$

Finally, since a normalized Kähler-Einstein weight is stationary for the nonnormalized Kähler-Ricci flow this finishes the proof of the theorem.

The last fiberwise elliptic equation in the previous corollary (in the $K_{X}$-setting) was first obtained by Schumacher [2008], who used the maximum principle to deduce an interesting consequence:

Corollary 4.8. Let $\pi: \mathscr{L} \rightarrow S$ be a fibration as in the previous theorem and assume that $K_{\mathscr{X} / S}$ is relatively ample. Then the canonical fiberwise Kähler-Einstein weight $\phi_{\mathrm{KE}}$ on $K_{\mathscr{X} / S}$ is smooth with semipositive curvature form on $\mathscr{X}$. Moreover, if the Kodaira-Spencer classes of the fibration are nontrivial for all s, then the curvature form of $\phi_{\mathrm{KE}}$ is strictly positive on $\mathscr{X}$.

The first part of the corollary was also shown by Tsuji [2006; 2011] using his iteration. Similarly, by a simple application of the parabolic maximum principle we deduce the following corollary from the parabolic equation in the previous theorem. 
Corollary 4.9. We make the same assumptions as in the previous theorem. Let $\phi_{t}$ evolve according to the Kähler-Ricci flow in the nonnormalized $\pm K_{X}$-setting. If the initial weight has (semi)positive curvature form on $\mathscr{L}$ then so has $\phi_{t}$ for all $t$. More precisely, $\left(d d^{c} \phi_{t}\right)_{x_{z}}>0$ (in all $n+1$ directions) at any point $x_{s}$ in the fiber $\mathscr{X}_{s}$ unless $\left(d d^{c} \phi_{0}\right)^{n+1}$ and $A_{\phi_{0}}$ vanish identically on $\mathscr{L}_{s}$.

Proof. As usual we may assume that $S$ is embedded in $\mathbb{C}$. Let us start with the semipositive case where the conclusion follows from the weak maximum principle. Indeed, assume to get a contradiction that $c\left(\phi_{t}\right) \geq 0$ on $\mathscr{X}$ for $t=0$ but that there is $(t, s, x)$ such that at $(t, s, x)$ we have $c\left(\phi_{t}\right)(s, x)<0$. By optimizing over $(x, t)$ we may also assume that $\partial\left(e^{a t} c\left(\phi_{t}\right)\right) / \partial t \leq 0, \Delta_{\omega_{t}^{X}} c\left(\phi_{t}\right) \geq 0$. Then (4-5) gives

$$
0 \geq e^{a t}\left(a c\left(\phi_{t}\right)+\frac{\partial c\left(\phi_{t}\right)}{\partial t}\right)=e^{a t}\left(\Delta_{\omega_{t}^{X}} c\left(\phi_{t}\right)-(a \pm 1) c\left(\phi_{t}\right)+\left|A_{\phi_{t}}\right|_{\omega_{t}^{X}}^{2}\right) .
$$

But if $a$ is chosen so that $a \pm 1>0$, the right-hand side above is strictly positive, giving a contradiction. To handle the remaining cases we invoke the following well-known strong maximum principle for the heat operator (which by standard argument can be reduced to the corresponding local statement in [Protter and Weinberger 1967]): let $h_{t} \geq 0$ satisfy

$$
\frac{\partial h_{t}}{\partial t} \geq \Delta_{g_{t}} h_{t} \quad \text { on }[0, T] \times X
$$

for any smooth family $g_{t}$ of Riemannian metrics. Then either $h_{t}>0$ for all $t>0$ or $h_{0} \equiv 0$. In our case we set $h_{t}=e^{a t} c\left(\phi_{t}\right)$ with $a= - \pm 1$ and conclude that if it is not the case that $c\left(\phi_{t}\right)>0$ for all $t>0$ then $c\left(\phi_{0}\right) \equiv 0$ and hence

$$
\frac{\partial}{\partial t} c\left(\phi_{t}\right)_{t=0}=\left|A_{\phi_{0}}\right|_{\omega_{0}^{X}}^{2}
$$

If we now assume, to get a contradiction, that the right-hand side above is strictly positive at $x_{0}$ then it follows that there is an $\epsilon>0$ such that $c\left(\phi_{t}\right)\left(x_{0}\right)>0$ for $t \in(0, \epsilon[$; that is, for such $t$ it is not the case that $c\left(\phi_{t}\right) \equiv 0$ on $X$. Hence, as explained above $c\left(\phi_{t}\right)>0$ on all of $] 0, \infty[\times X$, which yields the desired contradiction.

In particular, the previous corollary says that if the fibration $\mathscr{X}$ is infinitesimally nontrivial then the nonnormalized Kähler-Ricci flows instantly make any semipositively curved initial weight strictly positive.

Next we note that integrating the last formula in the previous theorem immediately gives the following corollary first shown by Fujiki and Schumacher [1990, Theorem 7.9].

Corollary 4.10. We make the same assumptions as in the previous theorem. Let $\phi_{\mathrm{KE}}$ be the weight of a smooth metric on $\pm K_{\mathscr{X} / S}$ which restricts to a normalized Kähler-Einstein weight on each fiber. Then

$$
\pi_{*}\left(\left(d d^{c} \phi_{\mathrm{KE}}\right)^{n+1} /(n+1) !\right)= \pm \omega_{\mathrm{WP}}
$$

on $S$, where $\pi_{*}$ denotes the fiber integral. In particular, if $S$ is effectively parametrized (i.e., all KodairaSpencer classes are nontrivial) then $\pm \pi_{*}\left(d d^{c} \phi_{-}\right)^{n+1}$ and hence the Weil-Petersson metric $\omega_{\mathrm{WP}}$ is a Kähler form on the base $S$. 
Remark 4.11. It follows immediately from the previous corollary that when $X$ is a Fano manifold the normalized Kähler-Einstein weight $\phi_{\mathrm{KE}}$ never has semipositive curvature on all of $\mathscr{X}$ if the family is effectively parametrized. Combining this fact with Corollary 4.9 shows that the relative Kähler-Ricci flow in the nonnormalized $K_{X}$-setting never converges in the $L^{1}(\mathscr{X})$-topology for an initial weight $\phi_{0}$ with semipositive curvature form on an effectively parametrized fibration $\mathscr{X}$.

4C. Quantization: The Bergman iteration. The (normalized) Bergman iteration in the $\pm K_{X}$-setting on $\mathscr{H}_{ \pm K_{X}}$ is defined precisely as in Section 3B, but using the probability measure $\mu_{ \pm}(\phi)$ in the definition of $\operatorname{Hilb}^{(k)}\left(\phi, \mu_{ \pm}(\phi)\right)$. Similarly, the nonnormalized Bergman iteration is defined in terms of the measure $\mu_{ \pm}^{\prime}$. The virtue of the nonnormalized setting is that the corresponding Hilbert norms correspond to the "adjoint" norms appearing in Berndtsson's Theorem 3.10:

$$
\operatorname{Hilb}^{(k)}\left(\phi, \mu_{\mu_{ \pm}^{\prime}}\right)(f, f):=i^{n^{2}} \int_{X} f \wedge \bar{f} e^{-(k \pm 1) \phi}:=\operatorname{Hilb}_{(k-1) L+K_{X}}(\phi)
$$

for $L= \pm K_{X}$. Moreover, they are clearly decreasing in $\phi$ (for $k \geq 1$ ) and hence the analogue of Proposition 3.13 of the corresponding Bergman iteration holds:

Proposition 4.12. Consider the Bergman iteration $\phi_{m}^{(k)}$ in the nonnormalized $\pm K_{X}$-setting and assume that $\phi_{m}^{(k)} \leq \psi_{m}^{(k)}$. Then $\phi_{m+1}^{(k)} \leq \psi_{m+1}^{(k)}$. Moreover, if $d(\phi, \psi)$ denotes the sup norm of $\phi-\psi$ then

$$
d\left(\psi_{m+1}, \phi_{m+1}\right) \leq d\left(\psi_{m+1}, \phi_{m+1}\right)\left(1 \pm \frac{1}{k}\right) .
$$

In particular, the Bergman iteration decreases the distance $d(\phi, \psi)$ in the nonnormalized $K_{X}$-setting.

Proof. Given the discussion preceding the proposition we just have to prove the claimed property of the distance $d$. But this follows directly from the monotonicity in the first part combined with the fact that $\log \rho^{(k)}\left(\phi_{m}+c\right) / k=\log \rho^{(k)}\left(\phi_{m}\right) / k- \pm \frac{c}{k}$, which in turn follows from $\mu_{ \pm}^{\prime}(\phi+c):=e^{ \pm(\phi+c)}=$ $\mu_{ \pm}^{\prime}(\phi) e^{ \pm c}$.

On the other hand, the following monotonicity of functionals holds in the normalized setting:

Lemma 4.13. The functionals $-I_{\mu_{ \pm}}$and $\mathscr{L}^{(k)}$ are increasing along the normalized Bergman iteration on $\mathscr{H}_{ \pm K_{X}}$. Moreover, they are strictly increasing at $\phi_{m}^{(k)}$ unless $\phi_{m}^{(k)}$ is stationary (when $k>1$ in the case of $\left.I_{\mu_{+}}\right)$.

Proof. By the general Lemma $2.6 \mathscr{L}^{(k)}$ is increasing and $I_{-}$is decreasing under the iteration, since $\phi \mapsto I_{-}(\phi)$ is concave with respect to the affine structure by Jensen's inequality. To show that $I_{+}^{(k)}$ is increasing in the $K_{X}$-setting just observe that

$I_{+}\left(\phi_{m+1}^{(k)}\right)-I_{+}\left(\phi_{m}^{(k)}\right):=\log \frac{\int e^{\left(\phi_{m+1}^{(k)}-\phi_{m}^{(k)}\right)} e^{\phi_{m}^{(k)}}}{\int e^{\phi_{m}^{(k)}}}=\log \int\left(\rho^{(k)}\right)^{\frac{1}{k}} \mu\left(\phi_{m}^{(k)}\right) \leq \log \left(\left(\int \rho^{(k)}\left(\phi_{m}^{(k)}\right) \mu\right)^{\frac{1}{k}}\right)=0$,

using Jensen's inequality applied to the concave function $t \mapsto t^{1 / k}$, which is strictly concave for $k>1$. 
Convergence of the Bergman iteration at a fixed level $k$.

Theorem 4.14. The Bergman iteration $\phi_{m}^{(k)}$ at level $k$ converges, when the discrete time $m \rightarrow \infty$, to a balanced weight $\phi_{\infty}^{(k)}$ in the following settings:

- the normalized $K_{X}$-setting;

- the normalized $-K_{X}$-setting if it is a priori assumed that there exists some balanced metric at level $k$ and $H^{0}(T X)=0$;

- the normalized $-K_{X}$-setting for $k$ sufficiently large under the assumption that $X$ admits a KählerEinstein metric and $H^{0}(T X)=0$;

- the nonnormalized $+K_{X}$-setting, where the limiting balanced weight is the unique normalized one.

Proof. Proof of the first point: By the previous lemma $-I_{\mu}$ is increasing and as shown in [Berman et al. 2009] $-\mathscr{F}_{\mu}^{(k)}$ is coercive (as follows immediately from Jensen's inequality). Moreover, as shown in [Berman et al. 2009] balanced weights are unique modulo scaling and hence all the convergence criteria in Proposition 2.9 are hence satisfied.

Proof of the second point: By the previous lemma $-I_{\mu}$ is increasing and as shown in [Berman et al. 2009] it follows immediately from Berndtsson's theorem (Theorem 3.10) applied to $L=-K_{X}$ that $-\mathscr{F}_{\mu}^{(k)}$ is strictly convex modulo scaling. Hence, the convergence follows by combining Proposition 2.9 and Lemma 2.10 .

Proof of the third point: The fact that $-\mathscr{F}_{\mu}^{(k)}$ is coercive was shown in [Berman et al. 2009] (using the corresponding coercivity of $-\mathscr{F}_{\mu}$ on $\mathscr{H}_{L}$ ). Given this coercivity the convergence follows as in the previous point.

Proof of the fourth point: Let $\left(\phi^{\prime}\right)_{m}^{(k)}=\phi_{m}^{(k)}+C_{m}^{(k)}$ denote the nonnormalized Bergman iteration in the $K_{X}$-setting. By the definition of the Bergman iteration (compare (4-10) below),

$$
\left(C_{m+1}^{(k)}-C_{m}^{(k)}\right)=-C_{m}^{(k)} / k-I\left(\phi_{m}^{(k)}\right) / k
$$

where by the first point above $I\left(\phi_{m}^{(k)}\right) \rightarrow I_{\infty}$ when $m \rightarrow \infty$. Set $D_{m}:=C_{m}^{(k)}+I\left(\phi_{m}^{(k)}\right)$. Then

$$
D_{m+1}=\left(1-\frac{1}{k}\right) D_{m}+\epsilon_{m},
$$

where $\epsilon_{m}=\left(I\left(\phi_{m+1}^{(k)}\right)-I\left(\phi_{m}^{(k)}\right)\right) \rightarrow 0$ as $m \rightarrow \infty$. It follows for elementary reasons that $D_{m} \rightarrow 0$; that is, $C_{m}^{(k)} \rightarrow-I_{\infty}$ showing that $\left(\phi^{\prime}\right)_{m}^{(k)}$ indeed converges and $I_{+}\left(\left(\phi^{\prime}\right)_{m}^{(k)}\right) \rightarrow 0$, proving the second point. For completeness we finally show that $D_{m} \rightarrow 0$. Assume for a contradiction that this is not the case. Then $D_{m+1} / D_{m} \rightarrow 1-\frac{1}{k}$ and hence $D_{m} \leq C_{\delta}\left(1-\frac{1}{k}+\delta\right)^{m} \rightarrow 0$ for $\delta$ sufficiently small, giving a contradiction.

The convergence in the fourth point above also follows immediately from the contracting property of the corresponding iteration (compare the proof of Theorem 4.20 below). We also note the following direct consequence of Berndtsson's theorem (Theorem 3.10), using formula (4-6) in the nonnormalized setting. 
Corollary 4.15. The Bergman iteration in the nonnormalized $\pm K_{X}$-setting preserves the (semi)positivity of the curvature of the initial weight. Moreover, if the fibration $\mathscr{X}$ is assumed infinitesimally nontrivial then any initial weight on $\pm K_{\mathscr{L} / S}$ which is semipositively curved and strictly positively curved along the fibers of $\mathscr{X}$ becomes strictly positively curved under the iteration.

Combining the previous corollary and Theorem 4.14 now gives the following:

Corollary 4.16. Let $\pi: \mathscr{L} \rightarrow S$ be a proper holomorphic submersion with $K_{\mathscr{x} / S}$ relatively ample. Let $\phi^{(k)}$ be the weight on $K_{\mathscr{X} / S}$ obtained by requiring that its restriction to any fiber is the unique normalized balanced weight at level $k$; i.e, $\int_{\mathscr{X}_{s}} e^{\phi^{(k)}}=1$. Then $\phi^{(k)}$ is smooth with semipositive curvature form. Moreover, if the fibration $\mathscr{X}$ is assumed infinitesimally nontrivial then $\phi^{(k)}$ is strictly positively curved.

Proof. Since positivity and smoothness are local notions it is enough to prove the corollary when $S$ is embedded in $\mathbb{C}$.

Smoothness: By definition $\phi^{(k)}=\mathrm{FS}^{(k)}\left(H^{(k)}\right)$ where $H^{(k)}$ is an element in the finite-dimensional smooth manifold $\mathscr{H}^{(k)}$ uniquely determined by $G^{(k)}\left(H^{(k)}, s\right)=0$ [Berman et al. 2009], where $G^{(k)}$ is the smooth map defined by

$$
G^{(k)}\left(H^{(k)}, s\right):=\left(T^{(k)}-I, I_{+} \circ \mathrm{FS}^{(k)}\right) \in \mathscr{H}^{(k)} \times \mathbb{R} .
$$

Moreover, as shown in [Berman et al. 2009] the linearization of $T^{(k)}-I$ is invertible modulo scaling (since it represents the differential of a functional on $H^{(k)}$ which is strictly convex modulo scaling). Hence, the claimed smoothness follows from the implicit function theorem.

Positivity: Since $K_{\mathscr{X} / S}$ is assumed relatively ample it admits a smooth weight $\phi_{0}$, which has fiberwise positive curvature form. After adding a sufficiently large multiple of the pull-back from the base of $|s|^{2}$ we may assume that $\phi_{0}$ has positive curvature over $\mathscr{X}$. By the last point of the previous theorem the Bergman iteration $\phi_{m}^{(k)}$ in the nonnormalized $K_{X}$-setting with initial weight $\phi_{0}$ yields a sequence of weights on $K_{\mathscr{X} / S}$ converging, when $m \rightarrow \infty$, uniformly to the unique normalized balanced weight $\phi^{(k)}$ at level $k$. As a consequence $d d^{c} \phi^{(k)} \geq 0$ on $\mathscr{X}$. Moreover, if the fibration $\mathscr{X}$ is assumed infinitesimally nontrivial the previous corollary shows that applying the Bergman iteration to $\phi^{(k)}$ yields a strictly positively curved metric. But since $\phi^{(k)}$ is fixed under the iteration this finishes the proof of the corollary.

Corollary 4.17. Let $\pi: \mathscr{L} \rightarrow S$ be the universal curve of the Teichmïller space of complex curves of a genus $g \geq 2$. Fix a positive integer $k$ (for $g=2$ we assume that $k \geq 2$ ). Under the natural isomorphism

$$
\left(T^{1,0} S\right)^{*}=\pi_{*}\left(2 K_{\mathscr{X} / S}\right)
$$

the fiberwise normalized balanced weight $\phi^{(k)}$ on $K_{\mathscr{x} / S}$ at level $k$ (appearing in the previous corollary) induces a Hermitian metric $\omega^{(k)}$ on $S$ with a curvature which is dually Nakano positive. Moreover, when $k \rightarrow \infty$ the metric $\omega^{(k)}$ converges towards the Weil-Petersson metric $\omega_{\mathrm{WP}}$ pointwise on $S$.

Proof. As is classical the assumptions on $k$ ensure that $K_{\mathscr{C} / S}$ is very ample. By the previous corollary $\phi^{(k)}$ is a smooth weight on $K_{\mathscr{X} / S} \rightarrow \mathscr{X}$ with strictly positive curvature and hence the $L^{2}$-metric on the direct 
image bundle $\pi_{*}\left(\mathscr{L}+K_{\mathscr{X} / S}\right)$ (with $\left.\mathscr{L}=K_{\mathscr{L} / S}\right)$ induced by $\phi^{(k)}$ has, according to the first point in Theorem 3.10, a curvature which is positive in the sense of Nakano. Since

$$
T^{1,0} S_{\mid s}=H^{1}\left(T^{1,0_{\mathscr{X}}}\right) \cong H^{0}\left(2 K_{\mathscr{X}_{s}}\right)^{*},
$$

this proves the first statement. To prove the pointwise convergence on $S$ of $\omega^{(k)}$ towards $\omega_{\mathrm{WP}}$ it is enough to prove that

$$
e^{-\phi^{(k)}} \rightarrow e^{-\phi_{\mathrm{KE}}}
$$

in $L_{\text {loc }}^{1}(X)$ for $X=\mathscr{L}_{s}$ (since, by definition, it implies the pointwise convergence of the corresponding Hermitian metrics on $\left.\pi_{*}\left(\mathscr{L}+K_{\mathscr{L} / S}\right)\right)$. But this convergence follows from the $L^{1}$ convergence of $\phi^{(k)}$ towards $\phi_{\mathrm{KE}}$ (Theorem 4.14) combined with the fact that $J\left(\phi^{(k)}\right)$ is uniformly bounded, as shown in [Berman et al. 2009] (see Lemma 6.4 therein). Alternatively, it follows immediately from the uniform convergence in Theorem 4.20 below.

The convergence in the previous corollary should be compared with the approximation results for the Weil-Petterson metric for moduli spaces of higher-dimensional manifolds recently obtained in [Keller and Lukic 2009]. The approximating Kähler metrics $\omega_{k}^{\prime}$ in that work are related to different balanced metrics, namely those defined with respect to Donaldson's original setting [2001] (where $\mu(\phi)=\operatorname{MA}(\phi)$ ).

Convergence towards the Kähler-Ricciflow.

Theorem 4.18. The following convergence results hold in all settings introduced in the beginning of Section 4 (i.e., in the (non)normalized $\pm K_{X}$-settings). Fix a smooth and strictly psh initial weight $\phi_{0}$ on $\pm K_{X}$ and consider the corresponding Bergman iteration $\phi_{m}^{(k)}$ at level $k$ and discrete time $m$, as well as the corresponding Kähler Ricciflow $\phi_{t}$. Then there is a constant A such that

$$
\sup _{X}\left|\phi_{m}^{(k)}-\phi_{m / k}\right| \leq A m / k^{2}
$$

uniformly in $(m, k)$ satisfying $m / k \leq T$ (in the $K_{X}$-setting $A$ is independent of $T$ ). In particular, if $m_{k}$ is a sequence such that $m_{k} / k \rightarrow t$, then $\phi_{m_{k}}^{(k)} \rightarrow \phi(t)$ uniformly on $X$ and

$$
d d^{c} \phi_{m_{k}}^{(k)} \rightarrow \omega_{t}
$$

on $X$ in the sense of currents, where $\omega_{t}$ evolves according to the corresponding Kähler-Ricci flow (4-2). The corresponding result also holds for the corresponding nonnormalized flows and in the relative setting, where the convergence is locally uniform with respect to the base parameter s.

Proof. In the case of the nonnormalized $K_{X}$-setting (denoted by primed objects) the proof of Theorem 3.15 carries over essentially verbatim, thanks to the last statement in Proposition 4.12 and Corollary 4.3 which gives the uniformity with respect to $T \in[0, \infty]$. To handle the nonnormalized $-K_{X}$-setting we need to modify the previous argument slightly. More precisely, we will prove that

$$
\sup _{X}\left|\phi_{m}^{(k)}-\phi_{m / k}\right| \leq A\left(1+\frac{1}{k}\right)^{m} m / k^{2} .
$$


Accepting this for the moment the claimed convergence when $m_{k} / k \rightarrow t$ follows using that

$$
\left(1+\frac{1}{k}\right)^{m}=\left(\left(1+\frac{1}{k}\right)^{k}\right)^{m / k} \leq e^{m / k} \leq e^{T}
$$

when $m / k \leq T$. To prove (4-8) first observe that Step 1 in the proof of Theorem 3.15 still applies for ( $m, k$ ) such that $m / k \leq T$ (using Proposition 2.11 applied to the nonnormalized $-K_{X}$-setting). In other words, there is a constant $A$ (depending on $T$ ) such that

$$
\sup _{X}\left|\psi_{k, m+1}-\left(\psi_{k, m}+F^{(k)}\left(\psi_{k, m}\right)\right)\right| \leq A\left(1 / k^{2}\right)
$$

for all $(m, k)$ such that $m / k \leq T$. Now we fix the integer $k$ and assume as an induction hypothesis that (4-7) holds for $m$ with $A$ the constant in the previous inequality. By Proposition 3.13,

$$
\begin{aligned}
\sup _{X}\left|\left(\psi_{k, m}+F^{(k)}\left(\psi_{k, m}\right)\right)-\left(\phi_{m}^{(k)}+F^{(k)}\left(\phi_{m}^{(k)}\right)\right)\right| & \leq \sup _{X}\left|\psi_{k, m}-\phi_{m}^{(k)}\right|\left(1+\frac{1}{k}\right) \\
& \leq\left(A\left(1+\frac{1}{k}\right)^{m} m / k^{2}\right)\left(1+\frac{1}{k}\right)
\end{aligned}
$$

with the same constant $A$ as above, using the induction hypothesis in the last step. Combining this estimate with the previous inequality gives

$$
\sup _{X}\left|\psi_{k, m+1}-\phi_{m+1}^{(k)}\right| \leq A\left(1+\frac{1}{k}\right)^{m+1} m / k^{2}+A / k^{2} .
$$

But using that $1 \leq\left(1+\frac{1}{k}\right)^{m+1}$ in the last term above proves the induction step and hence finishes the proof of the estimate (4-8).

To treat the Kähler-Ricci flows $\phi_{t}$ in the normalized settings we write

$$
\phi_{t}^{\prime}=\phi_{t}+C_{t},
$$

where $C_{t}$ is a constant for each $t$. Then

$$
\frac{\partial C_{t}}{\partial t}=-I_{ \pm}\left(\phi_{t}^{\prime}\right)
$$

Indeed, by the definition of the flow $\phi_{t}^{\prime}$ and $\phi_{t}$, we have

$$
\frac{\partial \phi_{t}^{\prime}}{\partial t}=\log \left(\mathrm{MA}\left(\phi_{t}^{\prime}\right)- \pm \phi_{t}^{\prime}\right), \quad \frac{\partial \phi_{t}}{\partial t}=\log \left(\mathrm{MA}\left(\phi_{t}\right)- \pm \phi_{t}\right)+ \pm I_{ \pm}\left(\phi_{t}\right)
$$

By scale invariance we may as well replace $\phi_{t}$ with $\phi_{t}^{\prime}$ on the right side of the second equation above and hence subtracting the second equation from the first one proves (4-9).

Similarly, writing

$$
\left(\phi^{\prime}\right)_{m}^{(k)}=\phi_{m}^{(k)}+C_{m}^{(k)},
$$

we obtain the following difference equation, using that the map $\phi \mapsto \rho^{(k)}(\phi)$, defined with respect to $\mu_{ \pm}$, is scale-invariant:

$$
C_{m+1}^{(k)}-C_{m}^{(k)}=-\frac{1}{k} I_{ \pm}\left(\left(\phi^{\prime}\right)_{m}^{(k)}\right)
$$


Now, as explained above, the estimate (4-7) holds for the primed objects and hence by the scaling equivariance of $I_{ \pm}$:

$$
\left|I_{ \pm}\left(\phi_{m / k}^{\prime}\right)-I_{ \pm}\left(\left(\phi^{\prime}\right)_{m}^{(k)}\right)\right| \leq A m / k^{2}
$$

A simple version of the argument given in the proof of Theorem 4.18 now shows, by comparing the differential equation (4-9) with the difference equation (4-10) and using (4-11), that

$$
\left|C_{m}^{(k)}-C_{m / k}\right| \leq B m / k^{2}
$$

for a uniform constant $B$. All in all this hence finishes the proof of the theorem.

We also have the following analogue of Corollary 3.16:

Corollary 4.19. For a fixed initial data $\phi_{0}=\phi_{0}^{(k)} \in \mathscr{H}_{ \pm} K_{X}$ the following convergence results hold for the Bergman iteration $\phi_{m}^{(k)}$ in the normalized $\pm K_{X}$-setting (in the $-K_{X}$-setting it is assumed that $H^{0}(T X)=0$ and $X$ a priori admits a Kähler-Einstein metric):

- For any sequence $m_{k}$ such that $m_{k} / k \rightarrow \infty$ the convergence $\phi_{m_{k}}^{(k)} \rightarrow \phi_{\infty}$ holds in the $L^{1}$-topology on $X$.

- The balanced weights $\phi_{\infty}^{(k)}:=\lim _{m \rightarrow \infty} \phi_{m}^{(k)}$ at level $k$ converge, when $k \rightarrow \infty$, in the $\mathscr{C}^{\infty}$-topology, to the weight $\phi_{\infty}$ which is the large time limit of the corresponding Kähler-Ricci flow.

Moreover, the convergence in the second point also holds in the nonnormalized $K_{X}$-setting, where the limit $\phi_{\infty}$ coincides with the canonical Kähler-Einstein weight $\phi_{\mathrm{KE}}$. In the relative case all convergence results hold fiberwise locally uniformly with respect to the base parameter s.

Proof. The proof of the first two points proceeds exactly as in the previous setting (again using the variational characterization in [Berman et al. 2009]). As for the claimed convergence in the nonnormalized setting it is obtained by noting that the large $m$ limit $\left(\phi^{\prime}\right)_{m}^{(k)}$ in the nonnormalized setting is the unique balanced weight such that $I_{ \pm}\left(\left(\phi^{\prime}\right)_{\infty}^{(k)}\right)=0$. In other words, $\left(\phi^{\prime}\right)_{\infty}^{(k)}=\phi_{\infty}^{(k)}-I_{ \pm}\left(\phi_{\infty}^{(k)}\right)$, where $\phi_{\infty}^{(k)}$ is the large $m$ limit of the iteration in the normalized setting. But by the second point above this means that $\left(\phi^{\prime}\right)_{\infty}^{(k)} \rightarrow \phi_{\infty}-I_{ \pm}\left(\phi_{\infty}\right)$ in $L^{1}$ (also using the continuity with respect to the $L^{1}$-topology of the functional $I_{ \pm}$on compacts; compare [Berman et al. 2009]). By uniqueness, this means that the limit must be $\phi_{\mathrm{KE}}$.

4D. Uniform convergence of the balanced weights in the $K_{X}$-setting. Next we point out that in the $K_{X}$-setting the convergence of the balanced weights is actually uniform (the proof is independent of the variational proof of a weaker convergence given in [Berman et al. 2009]). The proof simply uses that $\phi^{(k)}$ is close to $\phi_{t_{k}}$ where $\phi_{t}$ is the corresponding Kähler-Ricci flow and $t_{k}$ is a suitable sequence tending to infinity.

Theorem 4.20. Let $\phi^{(k)}$ be the balanced weight at level $k$ on the canonical line bundle $K_{X}$ (in the nonnormalized setting). When $k \rightarrow \infty$, the weights $\phi^{(k)}$ converge uniformly towards the normalized Kähler-Einstein weight $\phi_{\mathrm{KE}}$. 
Proof. Fix a smooth and positively curved weight $\phi_{0}$ on $K_{X}$ and denote by $\phi_{m}^{(k)}$ the Bergman iteration at level $k$ with initial data $\phi_{0}^{(k)}=\phi_{0}$. By Proposition 4.12 the map whose iterations define the Bergman iterations is a contraction mapping with contracting constant $q=\left(1-\frac{1}{k}\right)<1$ and hence it follows from the Banach fixed point theorem that

$$
\left\|\phi^{(k)}-\phi_{m}^{(k)}\right\|_{L^{\infty}} \leq \frac{q^{m}}{(1-q)}\left\|\phi_{1}^{(k)}-\phi_{0}\right\|_{L^{\infty}} .
$$

By definition we have $\phi_{1}^{(k)}-\phi_{0}=\frac{1}{k} \log \rho(k \phi)$, which, according to Proposition 2.11 , is uniformly bounded by a constant times $\frac{1}{k} \log k$; hence

$$
\left\|\phi^{(k)}-\phi_{m}^{(k)}\right\|_{L^{\infty}} \leq C\left(\left(1-\frac{1}{k}\right)^{k}\right)^{m / k} \log k .
$$

Next we take the sequence $m=m_{k}:=\left[k^{3 / 2}\right]$ where $[c]$ denotes the smallest integer which is larger than $c$. Then $t_{k}:=m_{k} / k=k^{1 / 2} \rightarrow \infty$ as $k \rightarrow \infty$ and since $\left(1-\frac{1}{k}\right)^{k} \rightarrow e^{-1}<1$ we conclude that

$$
\left\|\phi_{m}^{(k)}-\phi_{0}\right\|_{L^{\infty}} \rightarrow 0
$$

as $k \rightarrow \infty$. If now $\phi_{t}$ denotes the Kähler-Ricci flow in the nonnormalized $K_{X}$-setting we have, according to Theorem 4.18 , that

$$
\left\|\phi_{m}^{(k)}-\phi_{m_{k} / k}\right\|_{L^{\infty}} \rightarrow 0
$$

using that $m_{k} / k^{2} \rightarrow \infty$. Finally, since $\phi_{t_{k}} \rightarrow \phi_{\mathrm{KE}}$ uniformly as $t_{k} \rightarrow \infty$ this proves the theorem. Of course, the last convergence is not really needed for the proof as we may as well start with $\phi_{0}=\phi_{\mathrm{KE}}$ which is trivially fixed under the Kähler-Ricci flow.

It should be pointed out that the uniform convergence in the previous theorem has been previously obtained by Berndtsson (who also related it to Tsuji's iteration [Tsuji 2006]), using a different approach see the announcement in [Berndtsson 2009c]. But hopefully the relation to the convergence of the Kähler-Ricci flow above may shed some new light on the convergence.

4E. Families of varieties of general type and comparison with the NS metric. The quantized setting concerning the case when $K_{X}$ is ample admits a straightforward generalization to the case when $K_{X}$ is merely $\mathbb{Q}$-effective [Lazarsfeld 2004]. For simplicity we will only discuss the case when $K_{X}$ is big; that is, $X$ is a nonsingular variety of general type. Moreover, we will no longer assume that the map $\pi$ is a submersion. More precisely, we are given a surjective quasiprojective morphism $\pi: \mathscr{X} \rightarrow S$ between nonsingular varieties such that the generic fiber is a variety of general type. We denote by $S^{0}$ the maximal Zariski open subset of $S$ such that $\pi$ restricted to $\mathscr{X}^{0}:=\pi^{-1}\left(S^{0}\right)$ is a submersion, that is, a smooth morphism (and hence the fibers of $S^{0}$ are nonsingular varieties of general type).

Let us first consider the general absolute case, where we are given a line bundle $L \rightarrow X$ and an integer $k$ such that $k L$ is effective; that is, $H^{0}(X, k L) \neq\{0\}$. The main new feature in this more general setting is that any Bergman weight $\psi_{k}$ at level $k$, that is, $\psi_{k} \in \mathrm{FS}^{(k)}\left(\mathscr{H}^{(k)}\right)$, will usually have singularities; that is, it defines a singular metric on $L$ with positive curvature form. More precisely, the 
weight $k \psi_{k}$ on $k L$ is singular precisely along the base locus $B s(k L)$ of $k L$, that is, the intersection of the zero sets of all elements in $H^{0}(k L)$. Anyway, the difference of any two Bergman metrics is clearly bounded. Moreover, when $L=K_{X}$ the measure $\mu_{\psi_{k}}:=e^{\psi_{k}}$ has a smooth density which vanishes precisely along $B s(k L)$. As a consequence, we may fix such a reference (singular) weight $\phi_{0}:=\psi_{k}$ and the reference measure $\mu_{0}:=e^{\psi_{k}}$. Then Lemma 2.7 still applies (as explained in the remark following the lemma). As a consequence the proof of the convergence of the Bergman iteration to a balanced weight at level $k$ in the nonnormalized $K_{X}$-setting (Theorem 4.14) is still valid as long as $k K_{X}$ is effective. Combining this latter convergence with the generalizations [Berndtsson and Păun 2008b; 2008a] of Berndtsson's theorem (Theorem 3.10) and the invariance of plurigenera [Siu 1998] then gives the following generalization of Corollary 4.16:

Theorem 4.21. Let $\pi: \mathscr{X} \rightarrow S$ be a surjective quasiprojective morphism such that the generic fiber is a variety of general type. Then, for $k$ sufficiently large there is a unique singular weight $\phi^{(k)}$ on the relative canonical line bundle $K_{\mathscr{L} / S} \rightarrow \mathscr{X}$ with positive curvature current, such that the restriction of $\phi^{(k)}$ to any fiber over $S^{0}$ is a normalized and balanced weight at level $k$. Moreover, the weight $\phi^{(k)}$ is smooth on the Zariski open set defined as the complement in $\mathscr{X}$ of $\bigcup_{s \in S^{0}} B s\left(k K_{\mathscr{X}_{s}}\right) \cup \pi^{-1}\left(S-S^{0}\right)$.

Proof. Let us first prove the positivity statement. As before we may assume that $S$ is a domain in $\mathbb{C}$. First we consider the behavior over the set $S^{0}$, that is, where the fibration is a submersion. Fix $s_{0} \in S_{0}$ and write $X=\mathscr{Q}_{s_{0}}$. Let $\left(f_{i}\right)$ be a basis in $H^{0}\left(X, k K_{X}\right)$. By the invariance of plurigenera [Siu 1998] $s_{0}$ has a neighborhood $U \subset S_{0}$ with holomorphic sections $F_{i}$ of $k K_{\mathscr{L} / S} \rightarrow U$ such that $F_{i}$ restricts to $f_{i}$ on $X$. After perhaps shrinking $U$ we may hence assume that the restrictions of $F_{i}$ to any fiber give a basis in $H^{0}\left(\mathscr{C}_{S}, k K_{\mathscr{L}_{S}}\right)$. Now let $\phi_{0}:=\frac{1}{k} \log \left(\frac{1}{N_{k}} \sum\left|F_{i}\right|^{2}\right)$ so that $\phi_{0}$ is a singular weight on $K_{\mathscr{X} / S}$ over $U$ with positive curvature and such that $\phi_{0}$ restricts to a Bergman weight at level $k$ on each fiber. In particular,

$$
\int_{\mathscr{L}_{s}}|f|^{2} e^{-(k-1) \phi_{0}}<\infty
$$

for any $f \in H^{0}\left(\mathscr{C}_{S}, k K_{\mathscr{X}_{s}}\right)$. Decomposing, as before, $k K_{X}=(k-1) L+K_{X}$ with $L=K_{X}$, but now using Theorem 3.5 in [Berndtsson and Păun 2008b], shows that the curvature current of the weight $\phi_{1}^{(k)}:=\mathrm{FS}^{(k)} \circ \mathrm{Hilb}^{(k)}\left(\phi_{0}\right)$ on $K_{\mathscr{X} / S}$ is positive over $U$. Since, by definition, $\phi_{1}^{(k)}$ is still fiberwise a Bergman weight at level $k$ we may iterate the same argument and conclude that $\phi_{m}^{(k)}$ has a positive curvature current for any $m$. Now, as explained in the discussion before the statement of the theorem,

$$
m \rightarrow \infty \Longrightarrow \sup _{\mathscr{L}_{s}}\left|\phi_{m}^{(k)}-\phi^{(k)}\right| \rightarrow 0,
$$

locally uniformly with respect to $s$, where $\phi^{(k)}$ is the unique normalized fiberwise balanced weight at level $k$. In particular, it follows that $\phi^{(k)}$ has a curvature current which is positive over $S^{0}$.

To prove the claimed extension property of $\phi^{(k)}$ over $S-S_{0}$ first note that, writing $X=\mathscr{X}_{s}$ for a fixed fiber,

$$
\phi^{(k)} \leq \phi_{\mathrm{NS}}^{(k)}:=\log \left(\sup _{f \in H^{0}\left(X, k K_{X}\right)}\left(|f|^{2 / k} / \int_{X}(f \wedge \bar{f})^{1 / k}\right)\right),
$$


where $k \phi_{\mathrm{NS}}^{(k)}$ is the weight of the Narasimhan-Simha (NS) metric on $k K_{\mathscr{X} / S}$ [Narasimhan and Simha 1968; Kawamata 1982; Tsuji 2011; Berndtsson and Păun 2008a]. Accepting this for the moment we can use the result in [Berndtsson and Păun 2008a] saying that $\phi_{\mathrm{NS}}^{(k)}$ is locally bounded from above, with a constant which does not blow up as $s$ converges to a point in $S-S^{0}$ (this is proved by an $L^{2 / k}$ variant of the local Ohsawa-Takegoshi $L^{2}$-extension theorem). By the inequality (4-13) it hence follows that $\phi^{(k)}$ is also locally bounded from above by the same constant and then the claimed extension property follows from basic pluripotential theory.

Finally, to prove the inequality (4-13) fix a point $x \in X$. By the extremal definition of Bergman kernels there are sections $f_{i}$ (depending on $x$ ) such that

$$
\phi^{(k)}(x)=\frac{1}{k} \log \left(\frac{1}{N_{k}}\left|f_{1}\right|^{2}(x)\right) \quad \text { and } \quad \phi^{(k)}=\frac{1}{k} \log \left(\frac{1}{N_{k}} \sum_{i}\left|f_{i}\right|^{2}\right)
$$

on $X$. Since $\int_{X} e^{\phi^{(k)}}=1$ it hence follows that

$$
\int_{X}\left(\frac{1}{N_{k}} f_{1} \wedge \bar{f}_{1}\right)^{1 / k} \leq 1
$$

which finishes the proof of the inequality (4-13), since $f_{1} /\left(N_{k}\right)^{1 / 2}$ is a candidate for the supremum defining $\phi_{\mathrm{NS}}^{(k)}$.

As for the last smoothness statement in the theorem it is proved exactly as in Corollary 4.16, using that $\pi_{*}\left(k K_{\mathscr{K} / S}\right)$ is a locally trivial vector bundle over $S^{0}$. Indeed, it follows as before that the fiberwise normalized balanced metrics $H_{s}^{(k)}$, which by the local freeness may be identified with a family in $\operatorname{GL}\left(N_{k}\right)$, form a smooth family. Applying the Fubini-Study map to get $\phi^{(k)}$ then introduces the singular locus described in the statement of the theorem.

Remark 4.22. If one does not invoke the invariance of plurigenera in the proof of the previous theorem then the same argument gives the slightly weaker statement where $S^{0}$ is replaced by the intersection of $S^{0}$ with a Zariski open set where $\pi_{*}\left(k K_{\mathscr{L} / S}\right)$ is a locally trivial vector bundle. If one could then prove that the extension of $\phi^{(k)}$ is such that the integrability condition (4-12) holds over all of $S$, then the invariance of plurigenera would follow from a well-known version of the Ohsawa-Takegoshi extension theorem. It would be interesting to see if this approach is fruitful in the nonprojective Kähler case where the invariance of plurigenera is still open. When $\phi^{(k)}$ is replaced by the weight of the NS-metric $\phi_{\mathrm{NS}}^{(k)}$ (see formula (3-14)) this approach was used in [Tsuji 2011] to give a new proof of the invariance of plurigenera (in the projective case).

It should also be pointed out that (singular) Kähler-Einstein metrics and Kähler-Ricci flows have been studied recently for $K_{X}$ big. For example, using the deep finite generation of the canonical ring there is a unique Kähler-Einstein weight with minimal singularities which satisfies the Monge-Ampère equation

$$
\left(d d^{c} \phi_{\mathrm{KE}}\right)^{n} / n !=e^{\phi_{\mathrm{KE}}}
$$

on a Zariski open set in $X$ [Eyssidieux et al. 2009; Boucksom et al. 2010]. It seems likely that the positivity result in Corollary 4.8 can be extended to families of such singular weights $\phi_{\mathrm{KE}}$. But there are 
several regularity issues which need to be dealt with. Moreover, it also seems likely that the canonical balanced weights $\phi^{(k)}$ converge to $\phi_{\mathrm{KE}}$, when $K_{X}$ is big, but this would require a generalization of the convergence results in [Berman et al. 2009] (which only concern ample line bundles). This latter conjectural convergence should be compared with the convergence of the weight of the NS-metrics proved in [Berman and Demailly 2012], saying that $\phi_{\mathrm{NS}}^{(k)}$ converges in $L^{1}$ (and uniformly on compacts of an Zariski open set) to

$$
\phi_{\text {can }}:=\sup \left\{\psi: \int_{X} e^{\psi}=1\right\},
$$

where the sup is taken over all singular weights $\psi$ on $K_{X}$ with positive curvature current. In particular, $\phi_{\mathrm{KE}} \leq \phi_{\mathrm{can}}$, which is consistent with the inequality (4-13).

4F. Comparison with the constant scalar curvature and other settings. Given an ample line bundle $L \rightarrow$ $X$ the absolute setting when $\mu(\phi):=\left(d d^{c} \phi\right)^{n} / n$ ! was studied in depth by Donaldson [2001; 2005]. Of course, in this setting the Kähler-Ricci flow is trivial, but the corresponding quantized setting and the study of its large $k$ limit is highly nontrivial. In fact, it was shown in [Donaldson 2001] that, if it is a priori assumed that $c_{1}(L)$ contains a Kähler metric $\omega$ with constant scalar curvature and if $H^{0}(T X)=\{0\}$, then the curvature forms of any sequence of balanced weights converge in the $\mathscr{C}^{\infty}$-topology to $\omega$. Moreover, Donaldson showed that such balanced weights do exist for $k$ sufficiently large. As earlier shown by Zhang this latter fact is equivalent to the polarized variety $(X, k L)$ being stable in the sense of Chow-Mumford (with respect to a certain action of the group $\mathrm{SL}\left(N_{k}\right)$ ). An explicit proof of the convergence of the Bergman iteration in this setting was given in [Sano 2006] (see also [Donaldson 2005]).

Note that in this setting the functional $I_{\mu}$ is precisely the functional $\mathscr{E}$ (compare the beginning of Section 2). Since $\mathscr{E}$ is well-known to be concave on $\mathscr{H}_{L}$ with respect to the affine structure and $\mathscr{E} \circ \mathrm{FS}$ is geodesically convex on $\mathscr{H}^{(k)}$ the convergence of the corresponding Bergman iteration is also a consequence of Proposition 2.9.

It should also be pointed out that the role of the Kähler-Ricci flow of Kähler metrics in this setting is played by the Calabi flow. Indeed, as shown in [Fine 2010], the balancing flow, which is a continuous version of Donaldson's iteration, converges, at the level of Kähler metrics, in the large $k$ limit to the Calabi flow. More precisely, the balancing flow $H_{t}^{(k)}$ is simply the scaled gradient flow on the symmetric space $\mathscr{H}^{(k)}$ of the functional $\mathscr{F}^{(k)}$ in this setting and the convergence holds for the curvature forms of the weights $\mathrm{FS}^{(k)}\left(H_{t}\right)$ in $\mathscr{H}_{L}$.

Remark 4.23. Another, less studied, setting of geometric relevance (see [Berndtsson 2009b]) appears when we let

$$
\mu(\phi):=\frac{1}{N_{l}} \sum_{i=1}^{N_{l}} f_{i} \wedge \bar{f}_{i} e^{-l \phi}
$$

for a fixed integer $l$ where $f_{i}$ is an orthonormal basis for $H^{0}\left(l L+K_{X}\right)$ equipped with the Hermitian metric induced by $\phi$. When $L=-K_{X}$ and $l=1$ this is precisely the normalized $-K_{X}$-setting. In the general case $I_{\mu}(\phi)$ is essentially the induced metric on the top exterior power of the Hilbert space $H^{0}\left(l L+K_{X}\right)$. 
Moreover, as soon as the corresponding functional $\mathscr{F}_{\mu}^{(k)}$ has a critical point and $H^{0}(T X)=\{0\}$ the assumptions for convergence in Proposition 2.9 are satisfied (see [Berndtsson 2009b]).

\section{Acknowledgments}

I would like to thank Bo Berndtsson for interesting conversations on the topic of the present paper, in particular in connection to [Berndtsson 2009a; 2011]. In the first arXiv versions (1 and 2) of the present paper the very recent result about strict positivity of direct image bundles in [Berndtsson 2011] was not available, but in the present version it is used to improve the positivity properties of the Bergman iteration in the case of infinitesimally nontrivial fibrations. Also thanks to Valentino Tosatti and Yanir Rubinstein for comments on a draft of the present paper (in particular, thanks to Valentino Tosatti for pointing out the reference [Gross and Wilson 2000] in connection to semiflat metrics and the references in Remark 4.4, which was stated inaccurately in the previous version). Finally, thanks to the anonymous referee for helpful comments.

\section{References}

[Berman 2008] R. J. Berman, "Determinantal point processes and fermions on complex manifolds: Bulk universality", preprint, 2008. arXiv 0811.3341

[Berman and Boucksom 2010] R. Berman and S. Boucksom, "Growth of balls of holomorphic sections and energy at equilibrium", Invent. Math. 181:2 (2010), 337-394. MR 2011h:32021 Zbl 1208.32020

[Berman and Demailly 2012] R. Berman and J.-P. Demailly, "Regularity of plurisubharmonic upper envelopes in big cohomology classes", pp. 39-66 in Perspectives in analysis, geometry, and topology, Progress in Mathematics 296, Birkhäuser, New York, 2012. MR 2012m:32035 Zbl 06082161

[Berman et al. 2009] R. Berman, S. Boucksom, V. Guedj, and A. Zeriahi, "A variational approach to complex Monge-Ampère equations", preprint, 2009. arXiv 0907.4490

[Berndtsson 2009a] B. Berndtsson, "Curvature of vector bundles associated to holomorphic fibrations", Ann. of Math. (2) 169:2 (2009), 531-560. MR 2010c:32036 Zbl 1195.32012

[Berndtsson 2009b] B. Berndtsson, "Positivity of direct image bundles and convexity on the space of Kähler metrics", $J$. Differential Geom. 81:3 (2009), 457-482. MR 2010e:32020 Zbl 1187.53076

[Berndtsson 2009c] B. Berndtsson, "Remarks on a theorem by H. Tsuji", pp. 1107-1110 in Multiplier Ideal Sheaves in Algebraic and Complex Geometry (Oberwolfach, 2009), edited by S. Kebekus et al., Mathematisches Forschungsinstitut Oberwolfach 6, European Mathematical Society, Zürich, 2009.

[Berndtsson 2011] B. Berndtsson, "Strict and nonstrict positivity of direct image bundles", Math. Z. 269:3-4 (2011), 1201-1218. Zbl 05996694

[Berndtsson and Păun 2008a] B. Berndtsson and M. Păun, "A Bergman kernel proof of the Kawamata subadjunction theorem", preprint, 2008. arXiv 0804.3884

[Berndtsson and Păun 2008b] B. Berndtsson and M. Păun, "Bergman kernels and the pseudoeffectivity of relative canonical bundles”, Duke Math. J. 145:2 (2008), 341-378. MR 2009k:32020 Zbl 1181.32025

[Boucksom et al. 2010] S. Boucksom, P. Eyssidieux, V. Guedj, and A. Zeriahi, "Monge-Ampère equations in big cohomology classes", Acta Math. 205:2 (2010), 199-262. MR 2011k:32049 Zbl 1213.32025

[Bourguignon et al. 1994] J.-P. Bourguignon, P. Li, and S.-T. Yau, "Upper bound for the first eigenvalue of algebraic submanifolds", Comment. Math. Helv. 69:2 (1994), 199-207. MR 95j:58168 Zbl 0814.53040

[Cao 1985] H. D. Cao, "Deformation of Kähler metrics to Kähler-Einstein metrics on compact Kähler manifolds", Invent. Math. 81:2 (1985), 359-372. MR 87d:58051 Zbl 0574.53042 
[Chen 2000] X. Chen, "The space of Kähler metrics”, J. Differential Geom. 56:2 (2000), 189-234. MR 2003b:32031 Zbl 1041.58003

[Chen and Tian 2002] X. X. Chen and G. Tian, "Ricci flow on Kähler-Einstein surfaces", Invent. Math. 147:3 (2002), 487-544. MR 2003c:53095 Zbl 1047.53043

[Deligne 1987] P. Deligne, "Le déterminant de la cohomologie", pp. 93-177 in Current trends in arithmetical algebraic geometry (Arcata, CA, 1985), edited by K. A. Ribet, Contemporary Mathematics 67, American Mathematical Society, Providence, RI, 1987. MR 89b:32038 Zbl 0629.14008

[Donaldson 2001] S. K. Donaldson, "Scalar curvature and projective embeddings, I", J. Differential Geom. 59:3 (2001), 479-522. MR 2003j:32030 Zbl 1052.32017

[Donaldson 2005] S. K. Donaldson, "Scalar curvature and projective embeddings, II", Q. J. Math. 56:3 (2005), 345-356. MR 2006f:32033 Zbl 1159.32012

[Donaldson 2009] S. K. Donaldson, "Some numerical results in complex differential geometry", Pure Appl. Math. Q. 5:2 (2009), 571-618. MR 2010d:32020 Zbl 1178.32018

[Eyssidieux et al. 2009] P. Eyssidieux, V. Guedj, and A. Zeriahi, "Singular Kähler-Einstein metrics", J. Amer. Math. Soc. 22:3 (2009), 607-639. MR 2010k:32031 Zbl 1215.32017

[Fine 2010] J. Fine, “Calabi flow and projective embeddings", J. Differential Geom. 84:3 (2010), 489-523. MR 2012a:32023 Zbl 1202.32018

[Fujiki and Schumacher 1990] A. Fujiki and G. Schumacher, "The moduli space of extremal compact Kähler manifolds and generalized Weil-Petersson metrics", Publ. Res. Inst. Math. Sci. 26:1 (1990), 101-183. MR 92e:32012 Zbl 0714.32007

[Greene et al. 1990] B. R. Greene, A. Shapere, C. Vafa, and S.-T. Yau, "Stringy cosmic strings and noncompact Calabi-Yau manifolds", Nuclear Phys. B 337:1 (1990), 1-36. MR 91g:83057 Zbl 0744.53045

[Gross and Wilson 2000] M. Gross and P. M. H. Wilson, "Large complex structure limits of K3 surfaces", J. Differential Geom. 55:3 (2000), 475-546. MR 2003a:32042 Zbl 1027.32021

[Guedj and Zeriahi 2005] V. Guedj and A. Zeriahi, "Intrinsic capacities on compact Kähler manifolds", J. Geom. Anal. 15:4 (2005), 607-639. MR 2006j:32041 Zbl 1087.32020

[Hamilton 1982] R. S. Hamilton, "Three-manifolds with positive Ricci curvature”, J. Differential Geom. 17:2 (1982), 255-306. MR 84a:53050 Zbl 0504.53034

[Kawamata 1982] Y. Kawamata, “Kodaira dimension of algebraic fiber spaces over curves”, Invent. Math. 66:1 (1982), 57-71. MR 83h:14025 Zbl 0461.14004

[Keller 2009] J. Keller, “Ricci iterations on Kähler classes”, J. Inst. Math. Jussieu 8:4 (2009), 743-768. MR 2010h:32026 Zbl 1178.32020

[Keller and Lukic 2009] J. Keller and S. Lukic, "Numerical Weil-Petersson metrics on moduli spaces of Calabi-Yau manifolds", preprint, 2009. arXiv :0907.1387

[Koiso 1983] N. Koiso, "Einstein metrics and complex structures", Invent. Math. 73:1 (1983), 71-106. MR 85d:58018 Zbl 0515.53040

[Lazarsfeld 2004] R. Lazarsfeld, Positivity in algebraic geometry, I: Classical setting: Line bundles and linear series, Ergeb. Math. Grenzgeb. (3) 48, Springer, Berlin, 2004. MR 2005k:14001a Zbl 1093.14501

[Liu et al. 2008] K. Liu, X. Sun, and S.-T. Yau, "Good geometry on the curve moduli”, Publ. Res. Inst. Math. Sci. 44:2 (2008), 699-724. MR 2009h:32020 Zbl 1219.14012

[Mabuchi 1986] T. Mabuchi, “K-energy maps integrating Futaki invariants”, Tohoku Math. J. (2) 38:4 (1986), $575-593$. MR 88b:53060 Zbl 0619.53040

[Narasimhan and Simha 1968] M. S. Narasimhan and R. R. Simha, "Manifolds with ample canonical class", Invent. Math. 5 (1968), 120-128. MR 38 \#5253 Zbl 0159.37902

[Phong and Sturm 2004] D. H. Phong and J. Sturm, "Scalar curvature, moment maps, and the Deligne pairing", Amer. J. Math. 126:3 (2004), 693-712. MR 2005b:53137 Zbl 1077.53068

[Phong et al. 2007] D. H. Phong, N. Sesum, and J. Sturm, "Multiplier ideal sheaves and the Kähler-Ricci flow", Comm. Anal. Geom. 15:3 (2007), 613-632. MR 2009a:32037 Zbl 1143.53064 
[Phong et al. 2008] D. H. Phong, J. Song, J. Sturm, and B. Weinkove, "The Moser-Trudinger inequality on Kähler-Einstein manifolds", Amer. J. Math. 130:4 (2008), 1067-1085. MR 2009e:32027 Zbl 1158.58005

[Protter and Weinberger 1967] M. H. Protter and H. F. Weinberger, Maximum principles in differential equations, Prentice-Hall, Englewood Cliffs, NJ, 1967. MR 36 \#2935 Zbl 0153.13602

[Rubinstein 2008] Y. A. Rubinstein, "Some discretizations of geometric evolution equations and the Ricci iteration on the space of Kähler metrics", Adv. Math. 218:5 (2008), 1526-1565. MR 2009m:32045 Zbl 1143.53065

[Sano 2006] Y. Sano, "Numerical algorithm for finding balanced metrics", Osaka J. Math. 43:3 (2006), 679-688. MR 2008i: 32031 Zbl 1108.32014

[Schumacher 2008] G. Schumacher, "Positivity of relative canonical bundles for families of canonically polarized manifolds", preprint, 2008. arXiv 0808.3259

[Siu 1986] Y. T. Siu, "Curvature of the Weil-Petersson metric in the moduli space of compact Kähler-Einstein manifolds of negative first Chern class", pp. 261-298 in Contributions to several complex variables (Notre Dame, IN, 1984), edited by A. Howard and P.-M. Wong, Aspects Math. E9, Vieweg, Braunschweig, 1986. MR 87k:32042 Zbl 0598.32020

[Siu 1998] Y.-T. Siu, “Invariance of plurigenera”, Invent. Math. 134:3 (1998), 661-673. MR 99i:32035 Zbl 0955.32017

[Song and Tian 2009] J. Song and G. Tian, “The Kahler-Ricci flow through singularities”, preprint, 2009. arXiv 0909.4898

[Song and Tian 2012] J. Song and G. Tian, "Canonical measures and Kähler-Ricci flow”, J. Amer. Math. Soc. 25:2 (2012), 303-353. MR 2869020 Zbl 1239.53086

[Song and Weinkove 2010] J. Song and B. Weinkove, "Constructions of Kähler-Einstein metrics with negative scalar curvature", Math. Ann. 347:1 (2010), 59-79. MR 2011b:32037 Zbl 1205.53077

[Tian 1987] G. Tian, "Smoothness of the universal deformation space of compact Calabi-Yau manifolds and its Petersson-Weil metric", pp. 629-646 in Mathematical aspects of string theory (San Diego, CA, 1986), edited by S.-T. Yau, Adv. Ser. Math. Phys. 1, World Science Publishing, Singapore, 1987. MR 915841 Zbl 0696.53040

[Tian 2000] G. Tian, Canonical metrics in Kähler geometry, Birkhäuser, Basel, 2000. MR 2001j:32024 Zbl 0978.53002

[Tian and Zhu 2007] G. Tian and X. Zhu, “Convergence of Kähler-Ricci flow”, J. Amer. Math. Soc. 20:3 (2007), 675-699. MR 2007k:53107 Zbl 1185.53078

[Todorov 1989] A. N. Todorov, "The Weil-Petersson geometry of the moduli space of SU( $n \geq 3)$ (Calabi-Yau) manifolds, I", Comm. Math. Phys. 126:2 (1989), 325-346. MR 91f:32022 Zbl 0688.53030

[Tsuji 2006] H. Tsuji, "Dynamical construction of Kähler-Einstein metrics”, preprint, 2006. arXiv math/0606626

[Tsuji 2011] H. Tsuji, “Canonical singular Hermitian metrics on relative canonical bundles”, Amer. J. Math. 133:6 (2011), 1469-1501. MR 2863368 Zbl 05994418

[Voisin 2007] C. Voisin, Hodge theory and complex algebraic geometry, vol. 1, Cambridge Studies in Advanced Mathematics 76, Cambridge University Press, 2007. MR 2009j:32014 Zbl 1129.14019

[Wang 2005] X. Wang, "Canonical metrics on stable vector bundles”, Comm. Anal. Geom. 13:2 (2005), 253-285. MR 2006b: 32031 1108.32014 Zbl 1093.32008

[Yau 1978] S. T. Yau, "On the Ricci curvature of a compact Kähler manifold and the complex Monge-Ampère equation, I", Comm. Pure Appl. Math. 31:3 (1978), 339-411. MR 81d:53045 Zbl 0369.53059

[Yau 1987] S.-T. Yau, "Nonlinear analysis in geometry", Enseign. Math. (2) 33:1-2 (1987), 109-158. MR 88g:58003 Zbl 0631.53002

[Zelditch 2009] S. Zelditch, "Book review of Holomorphic Morse inequalities and Bergman kernels by Xiaonan Ma and George Marinescu; MR2339952”, Bull. Amer. Math. Soc. (N.S.) 46:2 (2009), 349-361. MR 2729895

Received 21 Aug 2011. Revised 15 Nov 2011. Accepted 20 Dec 2011.

ROBERT J. BERMAN: robertb@chalmers.se

Department of Mathematical Sciences, Chalmers University of Technology and the University of Gothenburg,

SE-SE-412 96 Göteborg, Sweden 


\title{
Analysis \& PDE
}

\author{
msp.org/apde
}

\section{EDITORS}

EDITOR-IN-CHIEF

\author{
Maciej Zworski \\ zworski@math.berkeley.edu \\ University of California \\ Berkeley, USA
}

BOARD OF EDITORS

\begin{tabular}{|c|c|}
\hline Michael Aizenman & $\begin{array}{l}\text { Princeton University, USA } \\
\text { aizenman@ math.princeton.edu }\end{array}$ \\
\hline Luis A. Caffarelli & $\begin{array}{l}\text { University of Texas, USA } \\
\text { caffarel@math.utexas.edu }\end{array}$ \\
\hline Michael Christ & $\begin{array}{l}\text { University of California, Berkeley, USA } \\
\text { mchrist@math.berkeley.edu }\end{array}$ \\
\hline Ursula Hamenstaedt & $\begin{array}{l}\text { Universität Bonn, Germany } \\
\text { ursula@math.uni-bonn.de }\end{array}$ \\
\hline Vaughan Jones & $\begin{array}{l}\text { University of California, Berkeley, USA } \\
\text { vfr@math.berkeley.edu }\end{array}$ \\
\hline Izabella Laba & $\begin{array}{l}\text { University of British Columbia, Canada } \\
\text { ilaba@math.ubc.ca }\end{array}$ \\
\hline László Lempert & $\begin{array}{l}\text { Purdue University, USA } \\
\text { lempert@math.purdue.edu }\end{array}$ \\
\hline Frank Merle & $\begin{array}{l}\text { Université de Cergy-Pontoise, France } \\
\text { Frank.Merle@u-cergy.fr }\end{array}$ \\
\hline Werner Müller & $\begin{array}{l}\text { Universität Bonn, Germany } \\
\text { mueller@math.uni-bonn.de }\end{array}$ \\
\hline Gilles Pisier & $\begin{array}{l}\text { Texas A\&M University, and Paris } 6 \\
\text { pisier@math.tamu.edu }\end{array}$ \\
\hline Igor Rodnianski & $\begin{array}{l}\text { Princeton University, USA } \\
\text { irod@ math.princeton.edu }\end{array}$ \\
\hline Sylvia Serfaty & $\begin{array}{l}\text { New York University, USA } \\
\text { serfaty@ cims.nyu.edu }\end{array}$ \\
\hline Terence Tao & $\begin{array}{l}\text { University of California, Los Angeles, US } \\
\text { tao@math.ucla.edu }\end{array}$ \\
\hline Gunther Uhlmann & $\begin{array}{l}\text { University of Washington, USA } \\
\text { gunther@math.washington.edu }\end{array}$ \\
\hline an Virgil Voiculesc & $\begin{array}{l}\text { University of California, Berkeley, USA } \\
\text { dvv@ math.berkeley.edu }\end{array}$ \\
\hline
\end{tabular}

Michael Aizenman

University of California, Berkeley, USA

University of California, Berkeley, USA

University of British Columbia, Canada

ilaba@math.ubc.ca

Université de Cergy-Pontoise, France

Merle@u-cergy.fr

William Minicozzi II

Yuval Peres

Tristan Rivière

Wilhelm Schlag

Yum-Tong Siu

Michael E. Taylor

András Vasy

Steven Zelditch

Sun-Yung Alice Chang

Charles Fefferman

Nigel Higson

Herbert Koch

Gilles Lebeau

Richard B. Melrose

Université Paris-Sud 11, France

nicolas.burq@math.u-psud.fr

Princeton University, USA

chang@math.princeton.edu

Princeton University, USA

cf@math.princeton.edu

Pennsylvania State Univesity, USA

higson@math.psu.edu

Universität Bonn, Germany

koch@math.uni-bonn.de

Université de Nice Sophia Antipolis, France lebeau@unice.fr

Massachussets Institute of Technology, USA rbm@math.mit.edu

Johns Hopkins University, USA

minicozz@math.jhu.edu

University of California, Berkeley, USA

peres@stat.berkeley.edu

ETH, Switzerland

riviere@math.ethz.ch

University of Chicago, USA

schlag@math.uchicago.edu

Harvard University, USA

siu@math.harvard.edu

Univ. of North Carolina, Chapel Hill, USA

met@math.unc.edu

Stanford University, USA

andras@math.stanford.edu

Northwestern University, USA

zelditch@math.northwestern.edu

PRODUCTION

production@msp.org

Silvio Levy, Scientific Editor

See inside back cover or msp.org/apde for submission instructions.

The subscription price for 2013 is US \$160/year for the electronic version, and \$310/year (+\$35, if shipping outside the US) for print and electronic. Subscriptions, requests for back issues from the last three years and changes of subscribers address should be sent to MSP.

Analysis \& PDE (ISSN 1948-206X electronic, 2157-5045 printed) at Mathematical Sciences Publishers, 798 Evans Hall \#3840, c/o University of California, Berkeley, CA 94720-3840, is published continuously online. Periodical rate postage paid at Berkeley, CA 94704, and additional mailing offices.

APDE peer review and production are managed by EditFLow ${ }^{\circledR}$ from Mathematical Sciences Publishers.

\section{PUBLISHED BY}

- mathematical sciences publishers

nonprofit scientific publishing

http://msp.org/

(C) 2013 Mathematical Sciences Publishers 


\section{ANALYSIS \& PDE Volume $6 \quad$ No. $1 \quad 2013$}

Some results on scattering for log-subcritical and log-supercritical nonlinear wave equations 1 HSI-WEI SHIH

Localisation and compactness properties of the Navier-Stokes global regularity problem

TERENCE TAO

A variational principle for correlation functions for unitary ensembles, with applications DORON S. LUBINSKY

Relative Kähler-Ricci flows and their quantization ROBERT J. BERMAN

Resolvent estimates for elliptic quadratic differential operators Michael Hitrik, Johannes SJÖSTRAND and Joe Viola

Bilinear Hilbert transforms along curves I: the monomial case XIAOCHUN LI

A gluing formula for the analytic torsion on singular spaces 